\title{
Equations d'évolution abstraites non linéaires de type parabolique $(*)$.
}

\author{
Giuseppe Da Prato (Trento) - Pierre Grisvard (Nice)
}

Summary. - We study abstract non linear parabolic equations by linearization and give examples to partial differential equations.

\section{1. - Introduction.}

1.1. Notre but est la résolution de l'équation différentielle

$$
u^{\prime}(t)=\varphi(t, u(t)), \quad t \in I
$$

ave la condition initiale

$$
u(0)=x
$$

où $I=[0, T]$ est un intervalle réel, en supposant que $\varphi$ est une application continûment dérivable au sens de Fréchet de $I \times D$ dans $E$ où $D$ et $E$ sont deux espaces de Banach tels que $D \subset E$ avee injection continue.

Les méthodes de monotonie développées dans Komura [15], Kato [14], CraNDall-Pazy [8], Crandall-Ligget [7] et Brezis [4], fourmissent une solution globale mais faible $d u$ problème (1.1.), (1.2.).

Dans le travail présent, on cherchera plutôt une solution classique. C'est-d-dire u continue $\grave{a}$ valeurs dans $D$ et continûment dérivable à valeurs dans $E$; cette solution sera éventuellement locale e'est-à-dire définie seulement dans $[0, \tau[$ avec $\tau<T$. Pour cela on utilisera une méthode de linéarisation proche de celle développée dans Sobolevsiki [21], Kato [14], Friedman [12], Sinestrari-Vernole [20], Pazy [19]. La différence essentielle par rapport aux travaux cités, est qu'on ne supposera pas ici que $(t, u) \mapsto \varphi(t, u)$ est une perturbation d'un opérateur linéaire (en $u)(t, u) \mapsto A(t) u$; ceci est rendu possible par l'introduction de nouveaux espaces d'interpolation.

1.2. Voici une brève description de la méthode employée, dans le cas particulier où l'équation (1.1.) est autonome c'est-à-dire où $\varphi$ ne dépend pas de $t \in I\left({ }^{1}\right)$. L'équa-

(*) Entrata in Redazione il 21 gennaio 1978.

(1) Cf. également la note Da Prato-Grisvard [10]. 
tion est done

$$
u^{\prime}(t)=\varphi(u(t)), \quad t \in I
$$

Dans ce qui suit, pour $X$ espace de Banach, $O([0, \tau] ; X)$ désignera l'espace des fonctions continues dans $[0, \tau]$ à valeurs dans $X$ muni de la norme

$$
u \rightarrow \max _{t \in[0, \tau]}\|u(t)\|_{x}
$$

Puisqu'on a supposé $\varphi$ continûment dérivable au sens de Fréchet de $D$ dans $E$, on peut introduire pour $x \in D$, l'élément $y=\varphi(x) \in E$ et l'opérateur $A=\varphi^{\prime}(x)$ qui est linéaire continu de $D$ dans $E$; on a alors le développement suivant de $\varphi$ autour de $x$

$$
\varphi(z)=y+A(z-x)+\Psi(z)
$$

où $\Psi$ est une fonction continûment dérivable au sens de Fréchet de $D$ dans $E$ avec en plus $\Psi(x)=0$ et $\Psi^{\prime}(x)=0$. Avec ces notations, on peut réecrire l'équation (1.3.) sous la forme

$$
u^{\prime}(t)-A u(t)=y-A w+\Psi(u(t)), \quad t \in I
$$

A ce point, il est naturel de supposer que $A$ est le générateur infinitésimal d'un semigroupe fortement continu dans $E$, de domaine $D_{A}=D$. Le problème s'écrit alors

c'est-à-dire

$$
u(t)=\exp [t A] x+\int_{0}^{t} \exp [(t-s) A](y-A x+\Psi(u(s))) d s
$$

$$
u(t)-\int_{0}^{t} \exp [(t-s) A] \Psi(u(s)) d s=x+(\exp [t A]-1)\left(A^{-1} y\right)
$$

en supposant, pour simplifier l'exposé introduetif, que $A$ est inversible.

Soit $S$ l'opérateur intégral qui intervient dans (1.4.) e'est-à-dire

$$
S u(t)=\int_{0}^{t} \exp [(t-s) A] \Psi(u(s)) d s .
$$

Si on se propose de résoudre l'équation (1.4.) par une méthode de point fixe dans $C([0, \tau] ; D)$, il est nécessaire que $S$ opère dans cet espace. L'application $u \rightarrow \Psi_{0} u$ est continue de $C([0, \tau] ; D)$ dans $C([0, \tau] ; E)$ et, par conséquent, demander que $B$ opère dans $O([0, \tau] ; D)$ revient à demander que

$$
f \mapsto \int_{0}^{\ell} \exp [(t-s) A] f(s) d s
$$


soit linéaire continu de $C([0, \tau] ; E)$ dans $C([0, \tau] ; D)$ ou encore, que la solution $v$ du problème

$$
\left\{\begin{array}{l}
v^{\prime}(t)-A v(t)=f(t), \quad t \in[0, \tau] \\
v(0)=0
\end{array}\right.
$$

soit dans $C\left([0, \tau] ; D_{A}\right)$ pour tout $f \in C([0, \tau] ; E)$.

Cette propriété n'est pas vraie en général, même si $A$ est générateur infinitésimal d'un semi-groupe analytique dans $E$ supposé Hilbertien.

En fait, on obtiendra la propriété désirée en remplaçant $E$ par un espace d'interpolation entre $D_{A}$ et $E$ et en supposant $t \rightarrow \exp [t A]$ analytique. Pour $0<\theta<1$, soit $D_{A}(\theta ; \infty)$ le sous-espace de $E$ formé des $x$ tels que

$$
t^{\theta} \cdot A(A-t)^{-1} x
$$

soit borné lorsque $t \rightarrow+\infty$. On munit cet espace de sa norme naturelle:

$$
x \rightarrow\|x\|_{\theta}=\|x\|_{E}+\sup _{t \geqslant 0}\left\|t^{\theta} A(A-t)^{-1} x\right\|_{E} .
$$

C'est un des espaces d'interpolation réelle entre $D_{A}$ et $E$, de Grisvard [13]. D'après DA Prato-Grisvard [9] cet espace a la propriété suivante $\left({ }^{2}\right)$ : pour tout $f \in C\left([0, \tau] ; D_{A}(\theta ;+\infty)\right)$ la solution $v$ du problème (1.5.) est telle que $A v \in C([0, \tau]$; $\left.D_{A}(\theta ;+\infty)\right)$ e'est-à-dire $v \in C\left([0, \tau] ; D_{A}(\theta+1 ;+\infty)\right)$ où on a posé $D_{A}(\theta+1 ;+\infty)=$ $=A^{-1} D_{A}(\theta ;+\infty)$. On en déduit que $S$ opère dans $C\left([0, \tau] ; D_{A}(\theta+1 ;+\infty)\right)$ à condition de supposer en plus que $\varphi$ (done aussi $\Psi$ ) est continûment différentiable au sens de Fréchet de $D_{A}(\theta+1 ;+\infty)$ dans $D_{A}(\theta,+\infty)$.

Du fait que $\Psi^{\prime}(x)=0$, on déduit que l'opérateur intégral $S$ est strictement contractant au voisinage de la fonction $u_{0}$ identiquement égale à $x$ dans $O([0, \tau]$; $\left.D_{A}(\theta+1 ;+\infty)\right)$ en supposant désormais que $s \in D_{A}(\theta+1 ;+\infty)$. Il en résulte que l'image de $1-S$ contient un voisinage de $u_{0}-S u_{0}=u_{0}$ dans $C\left([0, \tau] ; D_{A}(\theta+1 ;+\infty)\right)$ e'est-à-dire que l'équation (1.4.) admet une solution pourvu que

$$
\underset{0 \leqslant l \leqslant \tau}{\operatorname{Max}}\left\|(\exp [t A]-1)\left(A^{-1} y\right)\right\| D_{A}\left(\theta+1_{i}+\infty\right)
$$

soit assez petit. Il revient au même d'exiger que

$$
\exp [t A] y \rightarrow y
$$

lorsque $t \rightarrow 0$, dans $D_{d}(\theta ;+\infty)$ et que $\tau$ soit assez petit.

En conclusion, on a obtenu seulement un résultat d'existence locale et seulement pour $x$ tel que $y=\varphi(x)$ rende $\exp [t A]$ continu en $t=0$ dans la norme de $D_{A}(\theta ;+\infty)$.

(2) Cette propriété sera démontrée au $§ 2$. 
Cette dernière condition n'est pas automatiquement vérifiée par tout $y \in D_{A}(\theta ;+\infty)$ ear $D_{A}$ n'est pas en général dense dans $D_{A}(\theta ;+\infty)$.

On remplacera dans la suite $D_{A}(\theta ;+\infty) \operatorname{par} D_{A}(\theta)$ fermeture de $D_{A}$ dans $D_{A}(\theta ;+\infty)$; cet espace peut être redéfini par exemple comme sous-espace de $E$ formé des $x$ tels que

$$
t^{\theta} A(A-t)^{-1} x \rightarrow \vec{B}
$$

lorsque $t \rightarrow+\infty$.

A ce point, il est naturel de se demander si la dernière restriction imposée à $y$ est indispensable; en effet dans de nombreuses méthodes on montre que la norme de $S$ tend vers zéro lorsque $\tau \rightarrow 0$.

Ceci permet alors de résoudre (1.4.) sans restriction sur son second membre, à condition de supposer $\tau$ petit.

Cette technique est inapplicable ici. En effet, supposons en raisonnant par l'absurde, que la norme de $S$ comme opérateur continu dans $C\left([0, \tau] ; D_{A}(\theta+1, \infty)\right)$ tende vers zéro lorsque $\tau \rightarrow 0$; soit alors $x \in D_{A}(\theta+1, \infty)$ et $u$ la fonction constante égale à $x$. On a alors

$$
S u(t)=\int_{0}^{l} \exp [(t-s) A] \Psi(x) d s
$$

et

$$
A S u(t)=\int_{0}^{t} A \exp [(t-s) A] \Psi(x) d s=(\exp [t A]-1) \Psi(x)
$$

L'hypothèse implique alors

$$
\operatorname{Max}_{t \in[0, \tau]}\|(\exp [t A]-1) \Psi(x)\|_{D_{A}(\theta) \rightarrow 0}
$$

lorsque $\tau \rightarrow 0$, uniformément pour $\|\Psi(x)\|_{D_{A}(\theta)} \leqslant 1$; cela revient à dire que le semigroupe $\exp [t A]$ est uniformément continu et pas seulement fortement continu dans $D_{A}(\theta)$, ce qui n'est pas vrai en général (à moins que $A$ ne soit linéaire continu par. tout défini dans $E$ ).

1.3. L'idée centrale de ce travail étant ainsi esquissée, voici une brève explication du plan.

Le $\S 2$ est destiné à rendre la lecture de cet article aussi indépendante que possible de la théorie de l'interpolation. On introduit pour cela la méthode de l'interpolation continue qui même à la définition et la description des espaces $D_{A}(\theta)$ mentionnés plus haut.

Le $\$ 3$ est consacré essentiellement à la démonstration directe du résultat de régularité optimal pour les équations de la forme (1.5.) dans le cadre de l'espace des fonctions continues à valeurs dans $D_{A}(\theta)$. C'est, comme on l'a vu, le point de départ qui permet d'appliquer la méthode de linéarisation. Le cas où l'opérateur $A$ dépend 
régulièrement de $t$ est également traité. On donne enfin une démonstration du fait que le résultat optimal de régularité ne peut pas être obtenu dans le cadre de l'espace de départ $E$, même si celui-ci est hilbertien.

L'existence locale d'une solution de (1.1.) est prouvée au $\S 4$ où on suit le schéma esquissé en 1.2. On y démontre également qu'il existe une solution maximale dépendant continûment des données.

Le $\$ 5$ continue l'étude précédente dans le cas où par ailleurs on dispose d'une inégalité à priori pour la solution maximale de (1.1.): en gros, si la norme de $u(t)$ dans $D_{A}(\theta+1)$ reste bornée lorsque $t \rightarrow \tau$ borne supérieure de l'intervalle d'existence de $u$, alors $\tau=T$ e'est-à-dire que la solution maximale est globale.

On décrit au $\$ 6$, concrètement, les espaces $D_{A}(\theta)$ dans le cas particulier, essentiel dans les exemples, où $A$ est un opérateur élliptique réalisé avec des conditions aux limites dans un espace $L_{p}(\Omega)$ de fonctions de puissance $p$ intégrable $(1<p<\infty)$ dans $\Omega$ ouvert borné régulier de $\boldsymbol{R}^{n}$.

Le domaine $D_{A}$ est alors un espace de Sobolev avec conditions aux limites et les espaces $D_{A}(\theta)$ sont des espaces très voisins des espaces de NIKoLsKI [18] avec conditions aux limites.

Le $\$ 7$ montre comment la théorie du $\S 4$ s'applique aux problèmes mixtes relatifs aux équations paraboliques de la forme

$$
\frac{\partial u}{\partial t}=a(t, u, \nabla u, \Delta u)+f .
$$

Le point 7.1. considère le cas particulier où la dimension d'espace est un; on peut alors appliquer la théorie des $\$ \S 4$ et 5 sans faire appel aux espaces de Nikolski du $\S 6$. La lecture de ce point est done indépendante de celle du $\S 6$. Ensuite le point 7.2. considère le cas général et fait une consommation intensive d'espaces de Nikolski.

En 7.3. on considère le cas où la partie elliptique est en forme de divergence qui permet d'améliorer un peu les résultats de 7.2. En 7.4. on donne des résultats globaux.

Le § 8 développe enfin un exemple non susceptible d'être résolu par des méthodes à base de principe du maximum. Il s'agit d'un problème mixte relatif à l'équation parabolique (autonome pour simplifier) d'ordre quatre de la forme

$$
\frac{\partial u}{\partial t}=a\left(\Delta^{2} u\right)+f .
$$

Il n'a pas été possible, pour des raisons de brièveté de décrire tous les exemples de problèmes mixtes imaginables.

\section{2. - Espaces d'interpolation continue.}

2.1. Dans cette partie on introduit directement les espaces d'interpolation qui seront utilisés dans la suite, pour éviter de devoir se référer à la théorie générale 
de Lions-PeETre [16], dont on n'aurait à utiliser que les résultats les plus élémentaires. Les espaces introduits ici sont à rapprocher de ceux de Brezis-FraEnkel [5].

Soit $X, Y$ un couple d'espaces de Banach arec $Y \subset X{\left({ }^{3}\right)}^{3}$ (avec injection continue). Soit également $\theta \in] 0,1[$.

DéFInItion 2.1. - On désigne par $C(\theta, Y, X)$ l'espace des fonotions $t \rightarrow u(t)$ définies dans $] 0,1]$ à valeurs dans $Y$ et telles que

(i) $t \rightarrow t^{\theta} u(t)$ est continue de $[0,1]$ dans $Y$;

(ii) $t \rightarrow t^{\theta} u^{r}(t)$ est continue de $[0,1]$ dans $X$.

La dérivée de $u$ est entendue au sens des distributions dans $] 0,1[$ à valeurs dans $X$. On munit $C(\theta, Y, X)$ de la norme d'espace de Banach

$$
u \rightarrow \max _{0 \leqslant t \leqslant 1}\left\|t^{\theta} u(t)\right\|_{Y}+\max _{0 \leqslant t \leqslant 1}\left\|t^{\theta} u^{\prime}(t)\right\|_{X}=\|u\|_{C(\theta, Y, X)}
$$

Pour $u \in C(\theta, Y, X)$ on peut écrire si $s \leqslant t$,

$$
\|u(t)-u(s)\|_{X} \leqslant \int_{s}^{t}\left\|u^{\prime}(\sigma)\right\|_{X} d \sigma \leqslant\left(\int_{s}^{t} \frac{d \sigma}{\sigma^{\theta}}\right)\|u\|_{\alpha(\theta, Y, X)}
$$

et par conséquent $u$ est Hölderienne d'exposant $1-\theta$ à valeurs dans $X$, uniformément dans ]0,1]; ceci donne un sens à l'application $u \rightarrow u(0)$ linéaire continue de $O(\theta, Y, X)$ dans $X$. On peut ainsi poser la:

DÉfinimion 2.2. - On désigne par $(Y, X)_{\theta}$ l'espace décrit par $x=u(0)$ lorsque $u$ parcourt $C(\theta, Y, X)$.

C'est un espace de Banach pour la norme

$$
x \rightarrow \inf \|u\|_{C(\theta, Y, X)}=\|x\|_{\theta}
$$

où le inf, est pris par rapport à tous les $u \in C(\theta, Y, X)$ tels que $u(0)=x$.

Il est facile de vérifier les inclusions

$$
Y \subset(Y, X) c_{\theta}(Y, X)_{\theta}, X
$$

pour $0<\theta<\theta^{\prime}<1$, et la densité de $Y$ dans $(Y, X)_{\theta}$ pour $0<\theta<1$.

La propriété essentielle qui motive l'introduction de ces espaces est la propriété d'interpolation suivante pour les opérateurs linéaires continus: on considère deux

(3) Cette hypothèse n'est pas indispensable dans ce $\$ 2$ cependant pour les applications en vue dans ce travail il est inutile de se placer dans un cadre plus général. 
couples d'espaces de Banach $X_{1}, Y_{1}$ et $X_{2}, Y_{2}$ comme ci-dessus, e'est-à-dire que $\bar{Y}_{j} \subset X_{j}, j=1,2$ arec injection continue.

THÉORìme 2.3. - Soit II un opérateur linéaire continu de $X_{1}$ dans $X_{2}$, qui par restriction, est linéaire continu de $Y_{1}$ dans $Y_{2}$. Alors pour tout $\left.\theta \in\right] 0,1[, \Pi$ est linéaire continu de $\left(Y_{1}, X_{1}\right)_{\theta}$ dans $\left(Y_{2} ; X_{2}\right)_{\theta}$. De plus

$$
\|\Pi\|_{\left(Y_{1} ; X_{1}\right)_{\theta} \rightarrow\left(Y_{2} ; X_{2}\right)_{\theta}} \leqslant \operatorname{Max}\left\{\|\Pi\|_{Y_{1} \rightarrow Y_{2}} ;\|\Pi\|_{X_{1} \rightarrow X_{2}}\right\} .
$$

Démonstration. - Soit $x \in\left(Y_{1} ; X_{1}\right)_{\theta}$ alors d'après la définition 2.2., on a $x=u(0)$ avec $u \in C\left(\theta ; Y_{1}, X_{1}\right)$. Il est évident que $\Pi u \in C\left(\theta ; Y_{2}, X_{2}\right)$ donc $\Pi x=(\Pi u)(0) \in$ $\epsilon\left(Y_{2} ; X_{2}\right)_{\theta}$. Ceci prouve que $\Pi$ opère linéairement de $\left(Y_{1} ; X_{2}\right)_{\theta}$. Avec cette écriture la continuité de $\Pi$ et l'inégalité concernant les normes sont évidentes.

On peut énoncer un résultat analogue concernant les opérateurs non linéaires (ce n'est pas le plus fin possible dans cette direction).

THÉORÈME 2.4. - Soit II un opérateur continûment différentiable au sens de Fréchet de $X_{1}$ dans $X_{2}$, qui par restriotion, est uniformément Lipshitzien de $Y_{1}$ dans $Y_{2}$. Alors pour tout $\theta \in] 0,1\left[, \Pi\right.$ est continu de $\left(Y_{1}, X_{1}\right)_{\theta}$ dans $\left(Y_{2}, X_{2}\right)_{\theta}$.

Démonstration. - Soit $x \in\left(Y_{1}, X_{1}\right)_{\theta}$ alors $x=u(0)$ avee $u \in C\left(\theta ; Y_{1}, X_{1}\right)$. On a done $\Pi(x)=v(0)$ où $v=\Pi \circ u$.

On a vu plus haut que $u$ est continue dans $[0,1]$ à valeurs dans $X_{1}$ done $t^{\theta} x(t) \rightarrow 0$ dans $Y_{1}$ lorsque $t \rightarrow 0$.

Il est clair que $v$ est continue dans $] 0,1]$ à valeurs dans $X_{2}$; il faut étudier le comportement de $t^{\theta} v(t)$ lorsque $t \rightarrow 0$ : on sait que pour tout $\varepsilon>0$ il existe $\delta<0$ tel que

$$
0<t \leqslant \delta \Rightarrow\|u(t)\|_{Y_{1}} \leqslant \varepsilon / t^{\theta}
$$

On en déduit que

$$
\|v(t)-\Pi(0)\|_{Y_{2}}=\|\Pi(u(t))-\Pi(0)\|_{Y_{3}} \leqslant K \varepsilon / t^{\theta}
$$

pour $0<t \leqslant \delta$, où $K$ est la constante de Lipshitz de $\Pi$ opérateur de $Y_{1}$ dans $Y_{2}$. Il est clair que par conséquent, $t^{\theta} v(t) \rightarrow 0$ dans $\bar{Y}_{2}$ lorsque $t \rightarrow 0$. En conclusion $t^{\theta} v$ est continue dans $[0,1]$ à valeurs dans $Y_{1}$. Ensuite on a

$$
t^{\theta} v^{\prime}(t)=\Pi^{\prime}(u(t)) t^{\theta} u^{\prime}(t)
$$

Il est clair que $\Pi^{\prime} \circ u$ est continue dans $[0,1]$ à valeurs dans $L\left(X_{1} ; X_{2}\right)$ done $t^{\theta} v^{\prime}$ est continue dans $[0,1]$ à valeurs dans $X_{2}$. En conclusion $v \in C\left(\theta ; Y_{1}, X_{2}\right)$ et par conséquent $\Pi(x)=v(0) \in\left(Y_{2}, X_{2}\right)_{0}$. idées.

La démonstration de la continuité de $I I$ de $\left(Y_{1}, X_{1}\right)$ dans $\left(Y_{2}, X_{2}\right)_{\theta}$ suit les mêmes 
2.2. On va maintenant expliciter $(Y, X)_{\theta}$ dans un certain nombre de cas particuliers.

On commence par le cas où $Y$ est le domaine $D_{A}$ d'un opérateur $A$ linéaire fermé dans $X$, muni de la norme du graphe $\left({ }^{4}\right)$. Dans ce cas on pose simplement

$$
D_{A}(1-\theta)=\left(D_{A}, X\right)_{\theta} .
$$

On suppose pour le moment que $D_{A}$ est dense dans $X$; que $(A-t)$ est inversible pour tout $t>0$ et qu'il existe $C_{A}>0$ avec

$$
\left\|(A-t)^{-1}\right\|_{X \rightarrow X} \leqslant C_{A / t} .
$$

Sous ces hypothèses on a la caractérisation suivante de $D_{A}(\theta)$.

THÉOR亡̀me 2.5. $-D_{A}(\theta)$ est le sous-espace de $X$ formé des $x$ tels que $t^{\theta} A(A-t)^{-1} x \rightarrow 0$ dans $X$ lorsque $t \rightarrow+\infty$; de plus la norme de $D_{A}(\theta)$ est equivalente $\grave{a}$

$$
x \rightarrow\|x\|_{X}+\max _{t \geqslant 1}\left\|t^{\theta} A(A-t)^{-1} x\right\|_{X} .
$$

Démonstration. - Par définition de $D_{A}(\theta), x \in D_{A}(\theta)$ si et seulement si $x=u(0)$ où $t^{1-\theta} u(t)$ est continue dans $[0,1]$ à valeurs dans $D_{A}$ et $t^{1-\theta} u^{\prime}(t)$ est continue dans $[0,1]$ à valeurs dans $X$. On peut écrire par conséquent que

$$
x=u\left(\frac{1}{t}\right)-\int_{0}^{1 / t} u^{\prime}(s) d s
$$

pour $t>1$, d'où

$$
t^{\theta} A(A-t)^{-1} x=t(A-t)^{-1} \frac{1}{t^{1-\theta}} A u\left(\frac{1}{t}\right)-A(A-t)^{-1} t^{\theta} \int_{0}^{1 / t} u^{\prime}(s) d s .
$$

A ce point on remarque que grâce à (2.1.) et à la densité de $D_{A}$ dans $X$ on a:

$$
\begin{aligned}
& t(A-t)^{-1} \rightarrow-1 \\
& A(A-t)^{-1} \rightarrow 0
\end{aligned}
$$

fortement lorsque $t \rightarrow+\infty$, d'où

$$
\lim _{t \rightarrow+\infty} t^{\theta} A(A-t)^{-1} x=-\lim _{s \rightarrow 0} s^{1-\theta} A u(s)
$$

(') Dans toute la suite les domaines d'opérateurs considérés seront toujours munis de la norme du graphe sans que cela soit explicitement rappelé. 
ear

$$
\left\|t^{\theta} \int_{0}^{1 / t} u^{\prime}(s) d s\right\|_{X} \leqslant M t^{\theta} \int_{0}^{1 / t} \frac{d s}{s^{1-\theta}}=\frac{M}{\theta}
$$

où $M$ désigne le maximum de $\left\|t^{1-\theta} u^{\prime}(t)\right\|_{X}$ pour $t \in[0,1]$.

On a ainsi prouvé que pour $x \in D_{A}(\theta)$, la fonction $t^{\theta} A(A-t)^{-1} x$ reste bornée dans $X$ lorsque $t \rightarrow+\infty$ et de plus il existe une constante $K$ telle que

$$
\max _{t \geqslant 1}\left\|t^{\theta} A(A-t)^{-1} x\right\|_{X} \leqslant K\|x\|_{D_{A}(\theta)}
$$

D'autre part pour $x \in D_{A}$, on a évidemment

$$
\lim _{i \rightarrow+\infty} t^{\theta} A(A-t)^{-1} x=0
$$

Cette dernière propriété s'étend à tout $x \in D_{A}(\theta)$ par densité.

Réciproquement si $x \in X$ est tel que

$$
t^{\theta} A(A-t)^{-1} x \rightarrow 0
$$

dans $X$ lorsque $t \rightarrow+\infty$ alors posant

$$
u(t)=-\frac{1}{t}\left(A-\frac{1}{t}\right)^{-1} x
$$

on définit $u \in C\left(1-\theta ; D_{A}, X\right)$ tel que $u(0)=x$. C.Q.F.D.

2.3. A présent on restreint un peu les hypothèses sur $A$ en supposant que e'est le générateur infinitésimal d'un semi-groupe borné dans $X$, qu'on notera $t \rightarrow \exp [t A]$. L'hypothèse (2.1.) est alors vérifiée $C_{A}=\sup \|\exp [t A]\|_{X \rightarrow X}$. Sous ces hypothèses on a le

THÉonÈme 2.6. $-D_{A}(\theta)$ est le sous-espace de $X$ formé des $x$ tels que $t^{-\theta}(\exp [t A]-$ $-1) x \rightarrow 0$ dans $X$ lorsque $t \rightarrow 0$; de plus la norme de $D_{A}(\theta)$ est équivalente d̀

$$
x \mapsto\|x\|+\max _{0 \leqslant t \leqslant 1}\left\|t^{-\theta}(\exp [t A]-1) x\right\|_{x}
$$

Démonstration. - Par définition pour $x \in D_{A}(\theta)$, on peut écrire $x=u(0)$ où $t^{1-\theta} u(t)$ est continue à valeurs dans $D_{A}$ et $t^{1-\theta} u^{\prime}(t)$ continue à raleurs dans $X$. On peut écrire

$$
x=u(t)-\int_{0}^{t} u^{\prime}(s) d s, \quad \text { pour } t \leqslant 1
$$

22 - Annali di Matematica 
d'où

$$
t^{-\theta}(\exp [t A]-1) x=t^{-\theta} \int_{0}^{t} \exp [s A] A u(t) d s-(\exp [t A]-1) t^{-\theta} \int_{0}^{t} u^{\prime}(s) d s
$$

Comme $\exp [t A] \rightarrow 1$ fortement lorsque $t \rightarrow 0$, on en déduit que

$$
\lim _{t \rightarrow 0} t^{-\theta}(\exp [t A]-1) x=\lim _{t \rightarrow 0} t^{1-\theta} A u(t)
$$

car

$$
\left\|t^{-\theta} \int_{0}^{t} u^{\prime}(s) d s\right\|_{x} \leqslant M t^{-\theta} \int_{0}^{t} \frac{d s}{s^{1-\theta}}=\frac{M}{\theta},
$$

où $M$ désigne le maximum de $\left\|t^{1-\theta} u^{\prime}(t)\right\|_{X}$ pour $t \in[0,1]$.

On a ainsi obtenu que pour $x \in D_{A}(\theta)$, la fonction $t^{-\theta}(\exp [t A]-1) x$ est bornée dans $X$ lorsque $t \rightarrow 0$ et de plus il existe une constante $L$ telle que

$$
\max _{0 \leqslant i \leqslant 1}\left\|t^{-\theta}(\exp [t A]-1) x\right\|_{X} \leqslant L\|x\|_{D_{A}(\theta)}
$$

Pour $x \in D_{A}$, on a évidemment

$$
\lim _{t \rightarrow 0} t^{-0}(\exp [t A]-1) x=0,
$$

cette propriété s'étend à tout $x \in D_{A}(\theta)$ par densité.

Réciproquement si $x \in X$ est tel que

$$
t^{-\theta}(\exp [t A]-1) x \rightarrow 0
$$

dans $X$ lorsque $t \rightarrow 0$ alors en posant

$$
u(t)=\frac{1}{t} \int_{0}^{t} \exp [s A] x d s
$$

on obtient $u \in C\left(1-\theta ; D_{A}, X\right)$ tel que $u(0)=x$. En effet on a,

$$
A u(t)=\frac{1}{t}(\exp [t A]-1) x
$$

et par conséquent $t^{1-\theta} u(t)$ est continue à valeurs dans $D_{A}$. D'autre part on a

$$
\begin{aligned}
u^{\prime}(t) & =\frac{1}{t} \exp [t \cdot A] x-\frac{1}{t^{2}} \int_{0}^{t} \exp [s A] x d s \\
& =\frac{1}{t^{2}} \int_{0}^{t}(\exp [t \cdot A]-\exp [s A]) x d s
\end{aligned}
$$




$$
\begin{aligned}
& =\frac{1}{t^{2}} \int_{0}^{t}(\exp [t A]-\exp [(t-s) A]) x d s \\
& =\frac{1}{t^{2}} \int_{0}^{t} \exp [(t-s) A](\exp [s A] x-x) d s
\end{aligned}
$$

d'où

$$
\begin{aligned}
t^{1-\theta} u^{\prime}(t) & =\frac{1}{t} \int_{0}^{t} \exp [(t-s) A] \frac{\exp [s A] x-x}{s^{\theta}}\left(\frac{s}{t}\right)^{\theta} d s \\
& =\frac{1}{t} \int_{0}^{t} \exp [(t-s) A] \varphi(s)\left(\frac{s}{t}\right)^{\theta} d s
\end{aligned}
$$

avec $\varphi$ fonction continue à valeurs dans $X$ nulle en zéro. Il est clair que $t \mapsto t^{1-\theta} u^{\prime}(t)$ est continue dans ]0,1] et elle tend vers zéro lorsque $t \rightarrow 0$ car

$$
\left\|t^{1-\theta} u^{\prime}(t)\right\|_{x} \leqslant \frac{1}{t} \int_{0}^{t}\|\varphi(s)\|_{x} d s \rightarrow 0 . \quad \text { C.Q.F.D. }
$$

2.4. On passe maintenant à l'étude de l'espace $(Y ; X)_{\theta}$ dans le cas où $Y=\bigcap^{n} D_{A_{j}}$, où les $A_{j}, j=1,2, \ldots, n$ sont une famille d'opérateurs linéaires fermés dans $X{ }^{j=1}$

Techniquement dans une première étape, on supposera que $Y=D_{A} \cap Z$ où $Z$ est un second espace de Banach contenu dans $X$ avec injection continue et que $A$ vérifie (2.1.) et l'hypothèse supplémentaire suivante:

$$
Z \text { est stable par }(A-t) \mathbf{z}^{1} \text { pour tout } t>0 \text { et }
$$

$$
\left\|(A-t)^{-1}\right\|_{Z \rightarrow Z} \leqslant O_{A / t}
$$

On a alors le

Lemare 2.7. - On a l'identité

$$
\left(D_{A} \cap Z ; X\right)_{\theta}=\left(D_{A} ; X\right)_{\theta} \cap(Z ; X)_{\theta} .
$$

Démonstration. - L'inclusion du terme de gauche dans celui de droite résulte de la définition. Réciproquement soit $x \in\left(D_{A} ; X\right)_{\theta} \cap(Z ; X)_{\theta} ;$ on sait alors que $x=u(0)$ avee $u \in C(\theta ; Z, X)$ et que

$$
t^{1-\theta} A(A-t)^{-1} \not x \rightarrow 0
$$


dans $X$ lorsque $t \rightarrow+\infty$. On pose alors

$$
v(t)=-\frac{1}{t}\left(A-\frac{1}{t}\right)^{-1} u(t)
$$

on a $v(0)=u(0)=x$ et on va vérifier que $v \in C\left(\theta ; Z \cap D_{A}, X\right)$ ce qui achèvera de démontrer le lemme 2.7. Du fait que $t^{0} u(t)$ est continue à valeurs dans $Z, t^{\theta} v(t)$ a la même propriété grâce à (2.2.). On peut aussi écrire

$$
u(t)=x-\int_{0}^{t} u^{\prime}(s) d s
$$

d'où

$$
t^{\theta} A v(t)=-\frac{1}{t^{1-\theta}} A\left(A-\frac{1}{t}\right)^{-1} x+A\left(A-\frac{1}{t}\right)^{-1} t^{\theta}\left\{\frac{1}{t} \int_{0}^{t} u^{\prime}(s) d s\right\} .
$$

Par hypothèse sur $x$, le premier terme du second membre est continu dans $[0,1]$ à valeurs dans $X$ et d'autre part du fait que $t^{\theta} u^{\prime}(t)$ est continu dans $[0,1]$ à valeurs dans $X$, la fonction $t^{\theta}\left\{1 / t \int_{0}^{t} u^{\prime}(s) d s\right\}$ a la même propriété et par conséquent $t^{\theta} v$ est continu dans $[0,1]$ à valeurs dans $D_{A} \cap Z$.

Pour terminer on écrit que

$$
\begin{aligned}
& v^{\prime}(t)=-\frac{1}{t}\left(A-\frac{1}{t}\right)^{-1} u^{\prime}(t)-\frac{1}{t^{2}} A\left(A-\frac{1}{t}\right)^{-1} u(t) \\
&=-\frac{1}{t}\left(A-\frac{1}{t}\right)^{-1}\left\{u^{\prime}(t)+\frac{1}{t} A\left(A-\frac{1}{t}\right)^{-1} x\right\}-\frac{1}{t} A\left(A-\frac{1}{t}\right)^{-2}\left\{\frac{1}{t} \int_{0}^{t} u^{\prime}(s) d s\right\} ;
\end{aligned}
$$

en raisonnant comme ci-dessus on voit aisément que $t v^{\prime}$ est continu dans $[0,1]$ à valeurs dans $X$. C.Q.F.D.

Si maintenant on suppose que $Z=D_{B}$, il suffira que

$$
\left\|\left(B-t_{0}\right)(A-\lambda)^{-1}\left(B-t_{0}\right)^{-1}\right\|_{X \rightarrow X} \leqslant \theta / t
$$

pour tout $t>0$ et pour un $t_{0}$ dans l'ensemble résolvant de $B$, pour que (H) soit vérifié. On aura alors

$$
\left(D_{A} \cap D_{B} ; X\right)_{1-\theta}=D_{A}(\theta) \cap D_{B}(\theta) .
$$

C'est le cas en particulier si les résolvantes de $A$ et $B$ commutent; de cette remarque on déduit le

THÉonkime 2.8. - Soit $A_{1}, \ldots, A_{n}$ une famille d'opérateurs linéaires fermés dans $X$ 
GiUseppe Da Prato - Pierre Grisvard : Equations d'évolution abstraites, ete. 341

vérifiant tous (2.1.) alors si $\left[\left(A_{i}-t\right)^{-1} ;\left(A_{j}-t\right)^{-1}\right]=0$ pour tout $i$ et tout $j$ et $t>0$ et si on pose:

on a l'identité

$$
D_{A}=\bigcap_{j=1}^{n} D_{A_{j}}\left(^{(5)}\right.
$$

$$
\left(D_{A} ; X\right)_{1-\theta}=\bigcap_{j=1}^{n} D_{A_{j}}(\theta)
$$

Exemple 2.9. - Soit $X=L_{p}\left(\boldsymbol{R}^{n}\right)$ et $Y=W_{n}^{1}\left(\boldsymbol{R}^{p}\right)$ espace de Sobolev d'ordre un relatif à $L_{p}$ dans $\boldsymbol{R}^{n}$ alors l'espace $(Y ; X)_{1-\theta}$ est le sous-espace de $L_{p}\left(\boldsymbol{R}^{n}\right)$ formé des fonctions $u$ telles que

$$
\frac{1}{t^{\theta}}\left(\int_{\boldsymbol{R}^{n}}\left|u\left(x+t e_{j}\right)-u(x)\right|^{p} d x\right)^{1 / p} \rightarrow 0
$$

lorsque $t \rightarrow 0$ pour $j=1,2, \ldots, n$. Il suffit pour s'en convaincre d'appliquer les théorèmes 2.8 . et 2.6. avec $A j=\partial / \partial x_{j}$. L'espace ainsi obtenu est done la fermeture de $W_{n}^{1}\left(\boldsymbol{R}^{n}\right)$ dans l'espace de Nikolski $H_{p}^{(\theta)}\left(\boldsymbol{R}^{n}\right)$.

2.5. L'orsqu'on veut généraliser le résultat de cet exemple au cas où $Y$ est un espace de Sobolev d'ordre supérieur à un, on est naturellement conduit à expliciter $\left(D_{A^{m}}, X\right)_{1-\theta}$ toujours sous les mêmes hypothèses sur $A$. On a le résultat suivant:

THÉORÈME 2.10. - Si A vérifie (2.1.) alors

$$
D_{A^{m}}(\theta)=\left(D_{A^{k+1}} ; D_{A^{k}}\right)_{1-\sigma}
$$

oì lo est la partie entière de $m \theta$ et $\sigma$ sa partie fraotionnaire, à condition que mo ne soit pas entier.

EXEMPLE 2.11. - Soit $X=L_{p}\left(\boldsymbol{R}^{n}\right)$ et $Y=W_{p}^{m}\left(\boldsymbol{R}^{n}\right)$ espace de Sobolev d'ordre $m$ relatif à $L_{p}$ dans $\boldsymbol{R}^{n}$ alors pour $m \theta$ non entier, $m \theta=k+\sigma, k$ entier, $\left.\sigma \in\right] 0,1\left[,(Y ; X)_{1-\theta}\right.$ est le sous-espace de $W_{p}^{k}\left(\boldsymbol{R}^{n}\right)$ formé des $u$ telles que

$$
\frac{1}{t^{\sigma}}\left(\int_{\boldsymbol{R}^{\pi}}\left|D^{\alpha} u\left(x+t e_{j}\right)-D^{\alpha} u(x)\right|^{p} d x\right)^{1 / p} \rightarrow 0,
$$

lorsque $t \rightarrow 0$, pour tout multientier $\alpha$ de longueur $k$ et $j=1,2, \ldots, n$.

C'est donc la fermeture de $W_{p}^{m}\left(\boldsymbol{R}^{n}\right)$ dans l'espace de Nikolski $H_{p}^{(m \theta)}\left(\boldsymbol{R}^{n}\right)$. Ceci s'obtient en appliquant le théorème 2.10. avee $A=(\dot{1}-\Delta)^{\frac{1}{2}}$, le théorème 2.8 . et le théorème 2.6. avee $A_{j}=\partial / \partial x_{i}$.

(5) Muni de la norme somme des normes des graphes des $\boldsymbol{A}_{j}$. 
La démonstration du théorème 2.10. est fastidieuse; elle résultera de plusieurs lemmes:

LEMME 2.12. - $x \in(Y ; X)_{\theta}$ si et seulement si il existe un couple de fonctions $u$ et $v$ définies dans $] 0,1]$ telles que $x=u(t)+v(t)$ pout tout $t$ et

(i) $t \rightarrow t^{\theta} u(t)$ est continue de $[0,1]$ dans $Y$;

(ii) $t \rightarrow t^{\theta-1} v(t)$ est continue de $[0,1]$ dans $X$.

Démonstration. - Partant de la définition 2.1. la nécessité des conditions du lemme 2.10. est évidente en posant

$$
v(t)=-\int_{0}^{t} u^{\prime}(s) d s .
$$

Réciproquement si les conditions du lemme 2.12. sont remplies on a aussi $x=u_{1}(t)+$ $+v_{1}(t)$ où

et alors

$$
u_{1}(t)=\frac{1}{t} \int_{0}^{t} u(s) d s, \quad v_{1}(t)=\frac{1}{t} \int_{0}^{t} v(s) d s
$$

(i) $t^{\theta} u_{1} \quad$ est continu de $[0,1]$ dans $Y$;

(ii) $t^{\theta-1} v_{1}$ est continu de $[0,1]$ dans $X$.

On a nécessairement $v_{1}(0)=0$ done $x=u_{1}(0)$ et on conclut en observant que $u_{1} \in C(\theta ; Y, X)$ car $t^{\theta} u_{1}$ est continu à valeur dans $Y$ tandis que

$$
t^{\theta} u_{1}^{\prime}(t)=-t^{\theta} v_{1}^{\prime}(t)=-t^{\theta-1} v(t)+t^{\theta-1} v_{1}(t)
$$

donc $t^{\theta} u_{1}^{\prime}$ est continu à valeurs dans $X$. C.Q.F.D.

On introduit à présent une notion voisine de la classe $\varkappa_{\theta}$ de LIONS-PEETRE [16]:

Définitron 2.13. - Soit $Z$ un espaee de Banach tel que $Y \subset Z \subset X$, on dit que $Z$ est de classe $\theta$ entre $\bar{Y}$ et $X$ si

(i) il existe une constante $C$ telle que

$$
\|x\|_{Z} \leqslant C\|x\|_{Y}^{1-\theta}\|x\|_{X}^{\theta}
$$

pour tout $x \in X$

(ii) $Z$ est contenu aveo injeotion continue, dans $(Y ; X)_{0}$. 
Gruseppe Da Prato - Pierre Grisvard: Equations d'évolution abstraites, ete. 343

Il est élémentaire de vérifier que $Z=(Y, X)_{\theta}$ est de classe $\theta$ entre $Y$ et $X$; cependant pour la suite l'exemple suivant est plus important. On considère de nouveau un opérateur $A$ linéaire fermé dans $X$, vérifiant (2.1.)

Proposition 2.14. - Si A vérifie (2.1.), $D_{A}$ est de classe $\theta=1-k / m$ entre $D_{A^{m}}$ et $X$.

Démonstration. - Pour vérifier la propriété (i) de la défnition 2.13., il sufft de vérifier l'existence de $C_{m, k}$ telle que

$$
\left\|A^{k} x\right\|_{X} \leqslant O_{m, k}\|x\|_{X}^{1-k / m}\left\|A^{m} x\right\|_{X}^{k j m}
$$

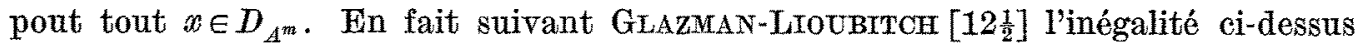
dans le cas général résulte de l'inégalité particulière avec $m=2$ et $k=1$ et

$$
O_{m, k} \leqslant\left(O_{2,1}\right)^{k\{m-k)}
$$

Pour vérifier l'existence de $C_{2,1}$, on écrit que pour $x \in D_{A}^{2}$ on a pour tout $t>0$ :

$$
A x=(A-t)^{-1} A^{2} x-t A(A-t)^{-1} x .
$$

Grâce à (2.1.) on déduit qu'il existe $M$ tel que

$$
\|A x\| \leqslant M\left(\frac{1}{t}\left\|A^{2} x\right\|+t\|x\|\right)
$$

pout tout $t>0$; le résultat suit en prenant le minimum par rapport à $t$.

Pour vérifier la propriété (ii) on se ramène au cas où $A$ est inversible en remplaçant eventuellement $A$ par $A-t_{0}$ avec $t_{0}>0$; alors on vérifie aisément par une suite d'intégrations par parties que

$$
x=(-1)^{m} \frac{(2 m-1) !}{[(m-1) !]^{2}} \int_{0}^{\infty} s^{m-1} A^{m}(A-s)^{-2 m} \nsim d s
$$

pour tout $\tau>0$ on peut donc écrire

$$
x=u(\tau)+v(\tau)
$$

où

$$
\left\{\begin{array}{l}
u(\tau)=(-1)^{m} \frac{(2 m-1) !}{[(m-1) !]^{2}} \int_{0}^{\tau} s^{m-1} A^{m}(A-s)^{-2 m} x d s \\
v(\tau)=(-1)^{m} \frac{(2 m-1) !}{[(m-1) !]^{2}} \int_{\tau}^{\infty} s^{m-1} A^{m}(A-s)^{-2 m} x d s .
\end{array}\right.
$$


344 GIUSEPPe Da Prato - PrezRe Grisyard: Equations d'évolution abstraites, ete.

Utilisant le fait que $x \in D_{A^{k}}$ on peut écrire grâce à (2.1.) que

$$
\begin{aligned}
& \left\|A^{m} u(\tau)\right\|_{X}=O\left(\int_{0}^{\tau} s^{n-k-1} d s\right)=O\left(\tau^{m-\bar{k}}\right), \\
& \|v(\tau)\|_{X}=O\left(\int_{\tau}^{\infty} s^{-1-\bar{k}} d s\right)=O\left(\tau^{-\bar{n}}\right) .
\end{aligned}
$$

On obtient le résultat espéré en posant $\tau=t^{-1 / m}$ c'est-à-dire $x=u\left(t^{-1 / m}\right)+v\left(t^{-1 / m}\right)$ et en remarquant que les fonctions $\tau \rightarrow \tau^{k-m} A^{m}(\tau)$ et $\tau^{k} v(\tau)$ sont continues en $\tau=+\infty$. C.Q.F.D.

L'intérêt essentiel des classes d'espaces introduites dans la définition 2.13. est la propriété de "réitération " suivante:

THÉorìne 2.15. - Soient $Z_{0}$ et $Z_{1}$ deux espaces de Banach, respectivement de classe $\theta_{0}$ et $\theta_{1}$ entre $\bar{Y}$ et $X\left({ }^{6}\right)$ alors $\left(Z_{0} ; Z_{1}\right)_{\theta}=(Y ; X)_{\omega}$ oì $\omega=(1-\theta) \theta_{0}+\theta \theta_{1}$ :

Le théorème 2.10. résulte directement des énoncés 2.14. et 2.15.

DÉmonstration. - On part de $x \in\left(Z_{0} ; Z_{1}\right)_{\theta}$; d'après le lemme 2.12. on a pour tout $t \in[0,1], x=u(t)+v(t)$ avee $t \rightarrow t^{\theta} u(t)$ continue de $[0,1]$ dans $Z_{0}$ et $t \rightarrow t^{\theta-1} v(t)$ continue de $[0,1]$ dans $Z_{1}$. De la définition 2.13. partie (ii) on déduit que

$$
\left\{\begin{array}{l}
u(t)=\alpha(t ; s)+\beta(t ; s) \\
v(t)=\gamma(t ; s)+\delta(t ; s)
\end{array}\right.
$$

pour tout $s \in[0,1]$ avec

$$
(t ; s) \rightarrow\left\{t^{\theta} s^{\theta_{0}} \alpha ; t^{\theta} s^{\theta_{0}-1} \beta ; t^{\theta-1} s^{\theta_{1}} \gamma ; t^{\theta-1} s^{\theta_{1}-1} \delta\right\}
$$

(') On suppose $\theta_{0}<\theta_{1}$, ce qui implique $Z_{0} \subset Z_{1}$. En effet si $x \in Z_{0}$, d'après le point (ii) de la définition 2.11. on a $x=u(0)$ où $u \in O(\theta ; Y, X)$. On a aussi $x=u_{1}(0)$ où

$$
u_{1}(t)=\frac{1}{t} \int_{0}^{t} u(s) d s .
$$

Il est facile de vérifier que $t^{\theta_{0}} u_{1}^{\prime}$ est continu de $[0,1]$ dans $X$ et $t^{\theta_{0}+1_{1}} u_{1}^{\prime}$ est continu de $[0,1]$ dans $Y$. D'autre part d'après la partie (i) de la définition 2.1. on a

d'où

$$
\left\|u_{1}^{I}(t)\right\|_{z_{1}} \leqslant C\left\|u_{1}^{i}(t)\right\|_{x}^{1-\theta_{1}}\left\|u_{1}^{I}(t)\right\|_{x}^{\theta_{s}}
$$

$$
\left\|u_{1}^{\prime}(t)\right\|_{z_{1}} \leqslant C_{1} / t^{1+\theta_{0}-\theta_{1}}
$$

la fonction $u_{1}^{\prime}$ est dono intégrable à valeurs dans $Z_{1}$ dès que $\theta_{0}<\theta_{1}$, d'où $u_{1}(0)=x \in Z_{1}$. 
continu de $[0,1] \times[0,1]$ dans $Y \times X \times Y \times X$. On obtient par conséquent la décomposition suivante de $x$ :

$$
x=u_{1}(t)+v_{1}(t)
$$

où

$$
\left\{\begin{array}{l}
u_{1}(t)=\alpha\left(t^{\theta_{1}-\theta_{0}} ; t\right)+\gamma\left(t^{\theta_{1}-\theta_{0}} ; t\right), \\
v_{1}(t)=\beta\left(t^{\theta_{1}-\theta_{0}} ; t\right)+\delta\left(t^{\theta_{1}-\theta_{0}} ; t\right) .
\end{array}\right.
$$

Il est élémentaire de vérifier que $t \rightarrow t^{\omega} u_{1}(t)$ est continue de $[0,1]$ dans $Y$ et $t \rightarrow t^{\omega-1} v_{1}(t)$ est continue de $[0,1]$ dans $X$ et par conséquent $x \in(Y ; X)_{\omega}$. Ceci prouve l'inclusion

$$
\left(Z_{0} ; Z_{1}\right) \subseteq(Y ; X)_{\omega}
$$

Pour prouver l'inclusion inverse, on part de $x \in(Y ; X)$ donc $x=u(0)$ avec $u \in C(\omega ; Y, X)$. La fonction $u_{1}$ définie par

$$
\left.\left.u_{1}(t)=\frac{1}{t} \int_{0}^{t} u(s) d s, \quad t \in\right] 0,1\right]
$$

vérifie également $x=u_{1}(0)$ et appartient aussi à $u \in C(\omega ; Y, X)$.

Cependant de l'identité

$$
\left.\left.u_{1}^{\prime}(t)=\frac{u(t)}{t}-\frac{1}{t^{2}} \int_{0}^{t} u(s) d s, \quad t \in\right] 0,1\right]
$$

on déduit qu'en outre $t \rightarrow t^{\omega+1} u_{1}^{\prime}(t)$ est continue de $[0,1]$ dans $Y$.

Par tronquature on peut aussi se ramener au cas où $u_{1}(\mathbf{1})=0$. On a done $x=v(t)+w(t)$ avec

$$
v(t)=-\int_{0}^{t} u_{1}^{\prime}(s) d s, \quad w(t)=-\int_{0}^{1} u_{1}^{\prime}(s) d s .
$$

La fonction $u_{1}^{\prime}$ prend ses valeurs dans $Y$ donc a fortiori dans $Z_{0}$ et $Z_{1}$; grâce à la première partie de la définition des classes $\theta_{0}$ et $\theta_{1}$ on déduit du fait que $t^{\omega+1} u_{1}^{\prime}$ et $t^{\omega} u_{1}^{\prime}$ sont continues de $[0,1]$ dans $Y$ et $X$ respectivement, que $t^{\omega+1-\theta_{0}} u_{1}^{\prime}$ et $t^{\omega+1-\theta_{1}} u_{1}^{\prime}$ sont continues de $[0,1]$ dans $Z_{0}$ et $Z_{1}$ respectivement ( $\left.{ }^{7}\right)$. Par intégration on en

( ${ }^{7}$ Le seul problème est en zéro mais comme $t^{\omega} u_{1}^{\prime} \rightarrow a$ dans $X$ on en déduit que $t^{\omega+1} u_{1}^{\prime}(t) \rightarrow 0$ dans $Y$ d'où $t^{\omega+1-\theta_{j}} u_{1}^{\prime}(t) \rightarrow 0$ dans $Z_{j}$ pour $j=0,1$. 
déduit que $t \rightarrow t^{\omega-\theta_{1}} v(t)$ est continue de $[0,1]$ dans $Z_{1}$ car $\theta_{1}>\omega$ et $t \rightarrow t^{\omega-\theta_{0}} w(t)$ est continue de $[0,1]$ dans $Z_{0}$ car $\omega>\theta_{0}$.

On conclut grâce au lemme 2.12. que $x \in\left(Z_{0}, Z_{1}\right)_{\theta}$ car $x=v\left(t^{\alpha}\right)+w\left(t^{\alpha}\right), t \in[0,1]$ avec

$$
\alpha=\frac{\theta}{\omega-\theta_{0}}=\frac{1-\theta}{\theta_{1}-\omega}
$$

(grâce à la relation $\left.\omega=(1-\omega) \theta_{0}+\omega \theta_{1}\right)$ et $t \rightarrow t^{\theta} w\left(t^{\alpha}\right)$ est continue de $[0,1]$ dans $Z_{0}$ et $t \rightarrow t^{\theta-1} v\left(t^{s}\right)$ est continue de $[0,1]$ dans $Z_{1}$. C.Q.F.D.

\section{3. - Equations linéaires.}

3.1. Cette partie est consacrée à l'utilisation des espaces $D_{A}(\theta)$ introduits en 2.2. dans l'étude de l'équation d'évolution

$$
\left.u^{\prime}(t)-A u(t)=f(t) \quad \text { dans } \quad I=\right] 0, T[
$$

avec la condition initiale $u(0)=x$. On suppose ici que $A$ est générateur infinitésimal d'un semi-groupe analytique fortement continu dans $E$ espace de Banach.

Pour être précis, on suppose que $(A-\lambda I)$ est inversible pour $|\arg \lambda|<\theta_{A}\left(\theta_{A}>\pi / 2\right)$ et que

$$
\left\|(A-\lambda I)^{-1}\right\|_{E \rightarrow E} \leqslant \frac{C_{A}(\theta)}{|\lambda|}
$$

pour arg $\lambda=\theta,|\theta|<\theta_{\Lambda}{ }^{\left({ }^{8}\right)}$.

Notre point de départ est le

THÉorìme 3.1. - Pour tout $f \in C\left([0, T] ; D_{A}(\theta)\right)$ et tout $x \in D_{A}(\theta+1)$ l'équation (3.1.) possède une unique solution $u \in C\left([0, T] ; D_{A}(\theta+1)\right)$ telle que $u(0)=x$, ̀̀ condition que $\theta \in] 0,1[$.

Ici on a posé par définition $D_{A}(\theta+1)=\left(D_{A^{2}} ; D_{A}\right)_{1-\theta}=\left\{u \in D_{A} ; A u \in D_{A}(\theta)\right\}$. Pour préciser toutes les constantes dans la suite, on utilisera sur $D_{A}(\theta)$ et $D_{A}(\theta+1)$ les normes suivantes:

$$
x \mapsto\|x\|_{\theta}=\|x\|_{E}+\max _{t \geqslant 1}\left\|t^{\theta} A(A-t)^{-1} x\right\|_{E} \quad \text { et } \quad x \mapsto\|x\|_{\theta+1}=\|x\|_{E}+\|A x\|_{\theta} .
$$

Démonstration. - La solution de l'équation (3.1.) vérifiant $u(0)=x$ s'écrit $u(t)=v(t)+w(t)$ où

$$
\begin{aligned}
& v(t)=\exp [t A] x, \\
& w(t)=\int_{0}^{t} \exp [(t-s) A] f(s) d s .
\end{aligned}
$$

${ }^{(8)}$ On supposera que $O_{A}$ est une fonction paire pour simplifier. 
L'étude de $v(t)$ est facile car les opérateurs exp $[t A]$ sont, par interpolation, équicontinus dans $D_{A}(\theta+1)$ pour $t$ décrivant tout intervalle borné de $[0,+\infty[$ et comme $D_{A}^{2}$ est dense dans $D_{A}(\theta+1), t \rightarrow \exp [t A]$ est fortement continu dans $D_{A}(\theta+1)$. En conséquence $v$ est continu dans $\left[0,+\infty\left[\right.\right.$ à valeurs dans $D_{A}(\theta+1)$ et

$$
\|v(t)\|_{\theta+1} \leqslant M\|x\|_{\theta+1} \quad \text { si } \quad\|\exp [t A]\|_{E \rightarrow E} \leqslant M .
$$

L'étude de $w(t)$ est l'essentiel de la démonstration.

On sait que

$$
\exp [t A]=-\frac{1}{2 i \pi} \int_{\gamma} \exp [t \lambda](A-\lambda I)^{-1} d \lambda
$$

où $\gamma$ est une courbe située dans le secteur $|\arg \lambda|<\theta_{A}$ et joignant $\infty \exp \left[-i \theta_{y}\right]$ à $\infty \exp \left[i \theta_{\gamma}\right]$ où $\pi / 2<\theta_{\gamma}<\theta_{A}$. On déduit que

$$
w(t)=-\frac{1}{2 i \pi} \int_{\gamma} \int_{0}^{t} \exp [(t-s) \lambda](A-\lambda I)^{-1} f(s) d s d \lambda .
$$

Sur cette identité on va d'abord vérifier que $w(t) \in D_{A}(\theta+1)$ il faut pour cela préciser la croissance de $(A-\lambda I)^{-1} f(s)$ le long de $\gamma$ :

LEMNe 3.2. - Pour $y \in D_{A}(\theta)$ et $|\arg \lambda|<\theta_{A}$ on a

$$
\left\|A(A-\lambda I)^{-1} y\right\|_{E} \leqslant\left\{1+2 O_{A}(|\arg \lambda|)\right\} \frac{\|y\|_{\theta}}{|\lambda|^{\theta}} .
$$

On admet provisoirement ce résultat. On a alors

puis

$$
\|w(t)\|_{E} \leqslant \int_{0}^{t} M\|f(s)\|_{E} d s \leqslant M T \operatorname{Max}_{t \in[0, T]}\|f(t)\|_{E}
$$

$$
A w(t)=-\frac{1}{2 i \pi} \int_{\gamma_{0}} \int_{0}^{t} \exp [(t-s) \lambda] A(A-\lambda)^{-1} f(s) d s d \lambda .
$$

Comme $f(s)$ est à valeurs dans $D_{A}(\theta)$ la majoration du lemme 3.2. implique que $A(A-\lambda I)^{-1} f(s)$ est intégrable au voisinage de $\lambda=0$; ainsi on peut déformer $\gamma$ en $\gamma_{0}$ contour formé des rayons issus de 0 et d'angles $+\theta_{\gamma}$, et $-\theta_{\gamma}$ avec l'axe réel positif. Il vient

$$
\begin{aligned}
\|A w(t)\|_{E} \leqslant \frac{1}{2 \pi} \int_{\gamma} \int_{0}^{t} \exp [(t-s) \operatorname{Re} \lambda]\left\{1+2 O_{A}\left(\theta_{\gamma}\right)\right\} \frac{\|f(s)\|_{\theta}}{|\lambda|^{\theta}} d s|d \lambda| \\
=\frac{1}{\pi} \int_{0}^{\infty} \int_{0}^{t} \exp \left[(t-s)\left(\cos \theta_{\gamma}\right) r\right]\left\{1+2 O_{A}\left(\theta_{\gamma}\right)\right\} \frac{\|f(s)\|_{\theta}}{r^{\theta}} d s d r
\end{aligned}
$$




$$
\begin{aligned}
& \leqslant \frac{1+2 C_{A}\left(\theta_{\gamma}\right)}{\pi\left|\cos \theta_{\gamma}\right|^{1-\theta}}\left(\int_{0}^{+\infty} \exp [-\varrho] \frac{d \varrho}{\varrho \theta}\right)\left(\int_{0}^{t} \frac{d s}{(t-s)^{1-\theta}}\right) \operatorname{Max}_{s \in[0, t]}\|f(s)\|_{\theta} \\
& \leqslant \frac{1+2 C_{A}\left(\theta_{\gamma}\right)}{\pi\left|\cos \theta_{\gamma}\right|^{1-\theta}}\left(\int_{0}^{+\infty} \exp [-\varrho] \frac{d \varrho}{\varrho \theta}\right) \frac{T^{\theta}}{\theta} \underset{t \in[0, T]}{\operatorname{Max}}\|f(t)\|_{\theta} .
\end{aligned}
$$

Enfin pour évaluer la norme de $w(t)$ dans $D_{A}(\theta+1)$ il faut encore majorer $A(A-$ $-\xi I)^{-1} A w(t)$. Pour cela utilisant le calcul fonctionnel de Dunford on remarque que au moins pour $f \in C\left([0, T] ; D_{A}\right)$ on a

$$
A(A-\xi I)^{-1} w(t)=-\frac{1}{2 i \pi} \int_{\gamma} \int_{0}^{t} \exp [(t-s) \lambda] \frac{\lambda}{\lambda-\xi}(A-\lambda I)^{-1} f(s) d s d \lambda
$$

donc en déformant le contour d'intégration on obtient

$$
\begin{aligned}
& \left\|A(A-\xi I)^{-1} A w(t)\right\|_{E} \leqslant \\
& \left.\frac{1}{\pi} \int_{0}^{+\infty} \int_{0}^{t} \exp \left[-(t-s)\left|\cos \theta_{\gamma}\right| r\right] \frac{r}{\left|r \exp \left[+i \theta_{\gamma}\right]-\xi\right|}\left\{1+20_{A} \theta_{\gamma}\right)\right\} \frac{\|f(s)\|_{\theta}}{r^{\theta}} d s d r \\
& \leqslant \frac{1}{\pi} \frac{1+2 \partial_{A}\left(\theta_{\gamma}\right)}{\cos \theta_{\gamma}} \int_{0}^{+\infty} \frac{d r}{\left|r \exp \left[ \pm i \theta_{\gamma}\right]-\xi\right| r^{\theta}} \operatorname{Max}_{0 \leq s \leq i}\|f(s)\|_{\theta} \\
& \leqslant\left(\frac{1}{\pi} \frac{1+2 O_{A}\left(\theta_{\gamma}\right)}{\cos \theta_{\gamma}} \int_{0}^{+\infty} \frac{d \varrho}{\left|\exp \left[ \pm i \theta_{\gamma}\right]-\varrho\right| \varrho^{\theta}}\right) \xi^{-\theta} \operatorname{Max}_{0 \leqslant t \leqslant T}\|f(t)\|_{\theta} .
\end{aligned}
$$

En réunissant toutes les majorations ainsi obtenues on voit que pour $f \in C\left([0, T] ; D_{A}\right)$ on a

$$
\|w(t)\|_{\theta+1} \leqslant K(\theta)(1+T)\left(1+C_{A}\left(\theta_{\gamma}\right)\right)\left(1+\frac{1}{\left|\cos \theta_{\gamma}\right|}\right) \operatorname{Max}_{t \in[0, T]}\|f(t)\|_{\theta} .
$$

Par densité il en résulte que pour $f \in C\left([0, T] ; D_{A}(\theta)\right)$, w est limite uniforme dans $D_{A}(\theta+1)$ de fonctions continues à valeurs dans $D_{A}(\theta+1)$ done $w \in C\left([0, T] ; D_{A}(\theta+1)\right)$ et la majoration indiquée reste vraie. C.Q.F.D.

ReMARQue 3.3. - L'inégalité obtenue est done

$$
\underset{0 \leqslant i \leqslant T}{\operatorname{Max}}\|u(t)\|_{\theta+1} \leqslant K(\theta)(1+T)\left(1+C_{A}\left(\theta_{\gamma}\right)\left(1+\frac{1}{\mid \cos \theta_{\gamma}}\right)\left\{\|x\|_{\theta+1}+\operatorname{Max}_{0 \leqslant t \leqslant T}\|f(t)\|_{\theta}\right\}\right.
$$

montre que même lorsque $T \rightarrow 0$ avec $x=0$ la costante ne tend pas vers zéro. Cela dépend du terme $\left\|A(A-\xi I)^{-1} A w(t)\right\|_{E}$ où on a en fait trouvé la meilleure 
majoration possible; en effet si $f(t)=y \in D_{A}(\theta)$ pour tout $t$ on a

$$
A w(t)=(\exp [t A]-1) y
$$

et par conséquent si la constante dans (3.3.) tendait vers zéro avee $T$ on aurait

$$
\operatorname{Max}_{0 \leqslant t \leqslant T} \frac{\|\exp [t A] y-y\|_{\theta}}{\|y\| \theta} \underset{T^{\prime \rightarrow 0}}{\longrightarrow} 0
$$

ceci impliquerait que $t \rightarrow \exp [t A]$ est uniformément continu dans $D_{A}(\theta)$ donc que $A$ est borné dans $D_{A}(\theta)$ (cf. DUNFord-Sohwarz [11]), ce qui n'est pas vrai en général.

Dámonstration du Lemie $3.2 .-a)$ Pour $|\lambda| \leqslant 1$ on écrit

$$
A(A-\lambda I)^{-1} y=y+\lambda(A-\lambda I)^{-1} y
$$

d'où grâce à (3.2.)

$$
\left\|A(A-\lambda I)^{-1} y\right\|_{E} \leqslant\left\{1+C_{A}(|\arg \lambda|)\right\}\|y\|_{E} \leqslant\left\{1+2 C_{A}(|\arg \lambda|)\right\} \frac{\|y\|_{\theta}}{|\lambda|^{\theta}} .
$$

b) Pour $|\lambda| \geqslant 1$ on écrit

$$
\begin{aligned}
A(A-\lambda I)^{-1} y= & \left\{A(A-\lambda I)^{-1}-|\lambda|(A-\lambda I)^{-1}\right\} \\
& A(A-|\lambda| I)^{-1} y
\end{aligned}
$$

et on en déduit que

$$
\left\|A(A-\lambda I)^{-1} y\right\|_{E} \leqslant\left\{1+2 C_{A}(|\arg \lambda|)\right\} \frac{\|y\|_{\theta}}{|\lambda|^{\theta}}
$$

grâce à la définition de la norme d'indice $\theta$.

Techniquement dans la suite on utilisera le résultat auxiliaire suivant:

Proposition 3.4. - Pour tout $f \in L^{\infty}\left([0, T] ; D_{A}(\theta)\right)$ et tout $x \in D_{A}(\theta+1)$ l'équation (3.1.) possède une unique solution $u \in C\left([0,1] ; D_{A}(\theta+1-\varepsilon)\right)$ telle que $u(0)=x$ à condition que $0<\varepsilon<\theta<1$.

La démonstration suit exactement les mêmes idées que celle du théorème 3.1.; les majorations sont plus simples grâce à l'exposant $\varepsilon>0$.

3.2. On va maintenant reprendre l'étude précédente dans le cas de l'équation plus générale

$$
\left.u^{\prime}(t)-A(t) u(t)=f(t) \quad \text { dans } \quad I=\right] 0, T[
$$


avee la condition initiale $u(0)=x$. On suppose que pour tout $t \in[0, T], A(t)$ est générateur infinitésimal d'un semi-groupe analytique fortement continu dans $E$, dépendant régulièrement de $t$. Plus précisément on suppose que

(i) $D_{A(t)}$ ne dépend pas de $t \in[0, T]$.

(ii) Il existe $\theta \in] 0,1\left[\right.$ tel que $D_{d(t)}(\theta+1)$ ne dépende pas de $t \in[0, T]$.

(iii) L'apptication $t \rightarrow A(t)$ est continue $\dot{a}$ valeurs dans $L\left(D_{A(0)} ; E\right)$.

(iv) L'application $t \rightarrow A(t)$ est continue d̀ valeurs dans $L\left(D_{A(0)}(\theta+1) ; D_{A(0)}(\theta)\right)$.

Sous les hypothèses (i) et (iii) on pent remarquer que les $A(t)$ sont «uniformément" générateurs infinitésimaux dans le sens du

Leñue 3.5. - Il existe $\theta_{A}$ et $O_{A}$ indépendants de $t$ tets que

$$
\left\|(A(t)-\lambda I)^{-1}\right\|_{E \rightarrow E} \leqslant \frac{O_{A}(\theta)}{|\lambda|}
$$

pour $|\arg \lambda|=\theta, 0 \leqslant \theta<\theta_{4}, t \in[0, T]$.

Démonstration. - Pour $|t-s|$ assez petit de sorte que, par exemple,

$$
\left\|(A(t)-A(s))(A(s)-I)^{-1}\right\|_{E \rightarrow E} \leqslant \frac{1}{2}
$$

pour tout $\lambda$ avec $\operatorname{Re} \lambda \geqslant 0, \lambda \neq 0$ on a

$$
(A(t)-\lambda I)^{-1}=(A(s)-\lambda I)^{-1}\left\{1+(A(t)-A(s))(A(s)-\lambda I)^{-1}\right\}^{-1}
$$

done

$$
\left\|(A(t)-\lambda I)^{-1}\right\|_{E \rightarrow E} \leqslant \frac{2 O_{A(s)}(\pi / 2)}{|\lambda|}
$$

La compacité de $[0, T]$ implique l'existence de $O$ tel que

$$
\left\|(A(t)-\lambda I)^{-1}\right\|_{E \rightarrow E} \leqslant \frac{C}{|\lambda|}
$$

pout tout $\lambda$ avec $\operatorname{Re} \lambda \geqslant 0, \lambda \neq 0$. Le Lemme 3.5 . suit par des arguments classiques (basés sur l'analyticité de la résolvante).

On en déduit facilement que les normes des espaces $D_{A(t)}(\theta)$ (et $\left.D_{A(t)}(\theta+1)\right)$ sont aussi uniformément équivalentes c'est-à-dire qu'il existe $M$ indépendant de $t$ et $s$ dans $[0, T]$ tel que

$$
\operatorname{Max}_{\xi \geqslant 1}\left\|\xi^{-\theta} A(t)(A(t)-\lambda I)^{-1} y\right\|_{E} \leqslant M \underset{\xi \geqslant 1}{\operatorname{Max}}\left\|\xi^{-\theta} A(s)(A(s)-\lambda I)^{-1} y\right\|_{E}
$$

pour tout $y \in D_{A(0)}(\theta)$. 
GIUseppe Da Prato - Pterre Grisvard: Equations d'évolution abstraites, ete. 351

THÉoRìme 3.6. - Pour tout $f \in C\left([0, T] ; D_{A(0)}(\theta)\right)$ et tout $x \in D_{A(0)}(\theta+1)$ l'équation (3.4.) possède une unique solution $u \in O\left([0, T] ; D_{A(0)}(\theta+1)\right)$ telle que $u(0)=x$.

Démonstration. - On utilise un raisonnement de perturbation à partir du résultat du Théorème 3.1.: On considère l'équation dans un intervalle éventuellement plus petit $[a, b]$; dans cet intervalle on réecrit. l'équation (3.4.) sous la forme

$$
u^{\prime}(t)-A(a) u(t)=f(t)+\{A(t)-A(a)\} u(t)
$$

et on pose $u(a)=y$. Comme $A(a)$ est un générateur infinitésimal, e'est équivalent à l'équation intégrale

$$
g(t)=f(t)+\{A(t)-A(a)\}\left[\exp [(t-a) A(a)] y+\int_{0}^{t} \exp [(t-s) A(a)] g(s) d s\right]
$$

où on a posé $g(t)=u^{\prime}(t)-A(a) u(t)$. Grâce au Théorème 3.1. l'équation (3,7.) est posée dans l'espace $C\left([a ; b] ; D_{A(\omega)}(\theta)\right)$ et elle possède une solution unique si l'opérateur

$$
T: g \rightarrow\{A(t)-A(a)\} \int_{0}^{t} \exp [(t-s) A(a)] g(s) d s
$$

est une contraction stricte. Pour vérifier cette dernière condition on introduit le module de continuité de $t \rightarrow A(t)$ considéré comme opérateur de $D_{A(0)}(\theta+1)$ dans $D_{\Delta(0)}(\theta)$

$$
\omega(\delta)=\underset{|t-s| \leqslant \delta}{\operatorname{Max}}\|A(t)-A(s)\|_{D_{A(0)}(\theta+1) \rightarrow D_{A(0)}(\theta)}
$$

On a alors

$$
\begin{aligned}
\operatorname{Max}_{a \leqslant t \leqslant b}\|T g(t)\|_{\theta} \leqslant \omega(b-a) \underset{a \leqslant t \leqslant b}{\operatorname{Max}}\left\|\int_{0}^{t} \exp [(t-s) A(a)] g(s) d s\right\|_{\theta+1} \\
\quad \leqslant \omega(b-a) K(\theta)(1+[b-a])\left(1+C_{A}\left(\theta_{\gamma}\right)\left(1+\frac{1}{\left|\cos \theta_{\gamma}\right|}\right) \underset{a \leqslant t \leqslant b}{\operatorname{Max}}\|g(t)\|_{\theta}\right.
\end{aligned}
$$

d'après l'inégalité (3.3.). On en déduit qu'il existe $\delta>0$ dépendant uniquement de $\omega$ et de $C_{A}\left(\theta_{\gamma}\right)$ et $\theta_{\gamma}$ tel que pour $b-a \leqslant \delta, T$ soit de norme $<1$ dans $C([a ; b]$; $\left.D_{A(\alpha)}(\theta)\right)$. En conséquence pour tout $y \in D_{A(0)}(\theta+1)$ et tout $f \in C\left([a, b] ; D_{A(0)}(\theta)\right)$ l'équation (3.7.) possède une solution unique $g$ dans $C\left([0, b] ; D_{A(0)}(\theta)\right)$ à condition que $b-a \leqslant \delta$. Grâce au Théorème 3.1. il revient au même de dire que l'équation (3.4.) possède une solution unique $u \in C\left([a, b] ; D_{d(0)}(\theta+1)\right)$ dans $[a, b]$, vérifiant $u(a)=y$. En recollant de telles solutions on obtient le Théorème 3.6. C.Q.F.D.

3.3. Voici pour terminer quelques remarques concernant le cas le plus farorable où $E$ est un espace de Hilbert. Les résultats mentionnés ici ont surtout pour 
röle de montrer la nécessité de l'introduction des espaces d'interpolation continue définis au $\S 1$.

On revient à la situation du point 3.1. Le problème considéré alors, au moins pour $T=+\infty$, équivaut, en posant

$$
\tilde{u}(t)= \begin{cases}0 & t<0 \\ u(t) & t>0\end{cases}
$$

à l'équation

$$
\tilde{u}^{\prime}(t)-A \tilde{u}(t)=\tilde{f}(t)+\infty \otimes \delta
$$

Le théorème de multiplicateurs de Dunford-Schwarz [11] permet de vérifier le

THÉORÈME 3.8. - Pout tout $f \in L^{p}([0, T] ; E)$ et tout $x \in D_{A}(1 / p ; p)$, il existe $u \in$ $\in L^{a}\left([0,1] ; D_{A}\right)$ unique solution de (3.1.) avee $u(0)=x$, pour $1<p<+\infty\left(^{(}\right)$.

Ensuite le même argument de perturbation qu'en 3.2. permet d'énoncer le

THÉORÈnE 3.9. - Sous les hypothèses (i) et (iii), pour tout $f \in L^{p}([0, T] ; E)$ et pour tout $x \in D_{A(0)}(1 / p ; p)$, il existe $u \in L^{p}\left([0, T] ; D_{A(0)}\right)$ unique solution de $(3.4)$ avec $u(0)=x$, pour $1<p<+\infty$.

Il est important de noter qu'il est impossible d'étendre ces énoncés au cas où on remplace les espaces $L^{p}$ par des espaces de fonctions continues munis de la norme du maximum. En effet, on a d'abord le résultat préliminaire suivant:

LEMre 3.10. - On suppose que $A$ est un générateur infinitésimal de semi-groupe analytique dans $E$, tel que pour tout $f \in C([0, T] ; E)$ l'équation (3.1.) admette une unique solution $u \in C\left([0, T] ; D_{A}\right)$ avec $u(0)=0$. Alors pour tout $f \in L^{\infty}([0, T] ; E)$ l'équation (3.1.) admet une unique solution $u \in C([0, T] ; E) \cap L^{\infty}\left([0, T] ; D_{A}\right)$ avec $u(0)=0$.

DÉmonstration. - On considère l'opérateur intégral $\delta$ qui résoud le problème:

$$
u(t)=(\delta f)(t)=\int_{0}^{t} \exp [(t-s) A] f(s) d s .
$$

Grâce au théorème du graphe fermé $A S$ est linéaire continu dans $C([0, T] ; E)$; soit $K$ sa norme. On considère également l'opérateur intégral $\sum$ qui résoud le problème adjoint soit

$$
v(t)=\left(\sum g\right)(t)=\int_{i}^{T} \exp \left[(s-t) A^{*}\right] g(s) d s
$$

${ }^{(9)} D_{A}(1 / p, p)$ est un espace d'interpolation réel défini su $\$ 6.2 . ;$ on peut ici se contenter de $x=0$. 
où

$$
\left\{\begin{array}{l}
\left.-v^{\prime}(t)-A^{*} v(t)=g(t), \quad t \in\right] 0, T[ \\
v(T)=0 .
\end{array}\right.
$$

Il est elair que $\sum$ est l'adjoint de $\delta$, de plus $\sum$ opère de $L^{1}([0, T] ; E)$ dans $L^{1}([0, T] ; E)$. En fait $A^{*} \sum$ est linéaire continu dans $L^{1}([0, T] ; E)$ : ceci se vérifie en remarquant que

$$
\underset{t \in[0 T]}{\operatorname{Max}}\left\|n(A-n I)^{-1} A S f(t)\right\|_{E} \leqslant K \operatorname{Max}_{i \in[0, T]}\|f(t)\|_{E}
$$

pour tout $n$. Ensuite on écrit que

$$
\begin{aligned}
\int_{0}^{T} \| n A^{*}\left(A^{*}\right. & -n I)^{-1} \sum g(t) \| d t \\
& =\left[\sup _{f \in C(0, T] ; E)}\left|\int_{0}^{T}\left(n A^{*}\left(A^{*}-n I\right)^{-1} \sum g(t) ; f(t)\right) d t\right| \operatorname{Max}_{t \in[0, T]}\|f(t)\|_{E} \leqslant 1\right] \\
& =\left[\sup _{f \in C([0, T] ; E)}\left|\int_{0}^{T}\left(g(t) ; n A(A-n I)^{-1} \mathbf{S} f(t)\right) d t\right| \operatorname{Max}_{t \in[0, T]}\|f(t)\|_{E} \leqslant 1\right] \leqslant K C_{A}(0) \int_{0}^{T}\|g(t)\|_{E} d t
\end{aligned}
$$

pout tout $n$, car $A(A-n I)^{-1}$ et $S$ commutent. On a donc à la limite (puisque $A^{*}$ est fermé) $\sum g \in L^{1}\left([0, T] ; D_{A^{*}}\right)$ et

$$
\int_{0}^{T}\left\|A^{*} \sum g(t)\right\| d t \leqslant K C_{A}(0) \int_{0}^{T} \| g(t)_{B} d t .
$$

Une nouvelle transposition montre que $A S$ est linéaire continu dans $L^{\infty}([0, T] ; E)$.

On en déduit la

Proposition 3.11. - Soit $A$ autoadjoint négatif dans $E$ tel que l'injection de $D_{A}$ dans $E$ soit compacte. Alors l'opérateur $f \mapsto A u$ où $u$ est solution du problème (3.1.) avec $u(0)=0$, n'est pas linéaire continu dans $C([0, T] ; E)$ quand $E$ est de dimension infinie.

Démonstratron. - On raisonne par l'absurde en supposant $f \rightarrow A u$ linéaire continu dans $C([0, T] ; E)$ donc aussi dans $L^{\infty}([0, T] ; E)$ grâce au Lemme 3.10 .

Le spectre de $A$ est discret, formé d'une suite de valeurs propres $\lambda_{i}, j=1,2, \ldots$ telles que $\lambda_{i} \searrow-\infty$. On choisit une suite de valeurs propres $\mu_{k}, k=1,2, \ldots$ telles que $\mu_{k+1}<2 \mu_{k}, \forall l_{k}$. Soient $\varphi_{k_{k}} \in D_{A}$ avee $A \varphi_{k}=\mu_{k} \varphi_{k},\left\|\varphi_{k}\right\|=1$ et $a_{k}=\mu_{k}^{-1}, k=1,2, \ldots$ La suite $a_{k}$ tend vers zéro en déeroissant.

Soit $f$ la fonction définie par

$$
f(t)= \begin{cases}\varphi_{k} & \text { pour } t \in\left[1-a_{k} ; 1-a_{k+1}[, k=1,2, \ldots\right. \\ 0 & \text { sinon }\end{cases}
$$

23 - Annali di Matematica 
Il est clair que $f \in L^{\infty}([0,1] ; E)$ que $\|f(t)\| \leqslant 1$ pour tout $t$. Soit $u$ la solution correspondante du problème (3.1.) avec $u(0)=0$, il est clair que

$$
\begin{aligned}
& u(t)=\int_{0}^{t} \exp [(t-s) A] f(s) d s= \\
& \sum_{k=1}^{n}\left(\int_{1-a_{k}}^{1-a_{k+1}} \exp \left[(t-s) \mu_{k}\right] d s\right) \varphi_{k}+\left(\int_{1-a_{n}}^{t} \exp \left[(t-s) \mu_{k}\right] d s\right) \varphi_{n}
\end{aligned}
$$

pour $1-a_{n+1} \geqslant t \geqslant 1-a_{n}$. On a pour les mêmes $t$

$$
A u(t)=\sum_{k=1}^{n}\left(\int_{1-a_{k}}^{1-a_{k+1}} \mu_{k} \exp \left[(t-s) \mu_{k}\right] d s\right) \varphi_{k}+\left(\int_{1-a_{n}}^{b} \mu_{n} \exp \left[(t-s) \mu_{n}\right] d s\right) \varphi_{n}
$$

d'où

$$
\begin{aligned}
\|A u(t)\|^{2} \geqslant \sum_{k=1}^{n}\left(\int_{1}^{1-a_{k}} \mu_{k+1} \exp \left[(t-s) \mu_{k}\right] d s\right)^{2} & \\
& =\sum_{k=1}^{n}\left(\exp \left[\left(t-1+a_{k}\right) \mu_{k}\right]-\exp \left[\left(t-1+a_{k+1}\right) \mu_{k}\right]\right)^{2} .
\end{aligned}
$$

Par hypothèse on a\| $\|A u(t)\| \leqslant K O_{A}(0)$ presque partout d'où

$$
\sum_{k=1}^{n}\left(\exp \left[\left(t-1+a_{k}\right) \mu_{k}\right]-\exp \left[\left(t-1+a_{k+1}\right) \mu_{k}\right]\right)^{2} \leqslant K^{2} C_{A}^{2}(0)
$$

pour $1-a_{n} \leqslant t \leqslant 1$ et pour tout $n$. A la limite lorsque $t \rightarrow 1$ et $n \rightarrow+\infty$ on obtient la convergence de la série

$$
\sum_{k \geqslant 1}\left(\exp \left[\mu_{k} a_{k}\right]-\exp \left[\mu_{\varepsilon} a_{k+1}\right]\right)^{2}
$$

Cependant on a

$$
\begin{aligned}
\left|\exp \left[\mu_{k} a_{k}\right]-\exp \left[\mu_{k} a_{k+1}\right]\right| & =\exp [-1]\left|1-\exp \left[\mu_{k}\left[a_{k+1}-a_{k}\right]\right]\right| \\
& =\exp [-1]\left|1-\exp \left[\frac{\mu_{k}}{\mu_{k+1}}-1\right]\right| \geqslant \exp [-1]\left|1-\exp \left[-\frac{1}{2}\right]\right|
\end{aligned}
$$

ce qui établit la contradiction.

REMaRque 3.12. - On peut se débarrasser facilement de l'hypothèse que l'injection de $D_{A}$ dans $E$ est compacte.

Cependant l'énoncé ci-dessus étant certainement vrai dans des hypothèses beaucoup plus générales, on se limite à la Proposition 3.11. 


\section{4. - Equations non linéaires.}

4.1. On établit ici l'existence d'une solution locale pour nne équation de la forme (1.1.).

Pour cela on fixe une fois pour toutes, un opérateur linéaire fermé $A$ de domaine $D_{A}$ dans $E$. On suppose que $A$ vérifie (2.1.) de manière à pouvoir considérer les espaces d'interpolation $D_{A}(\theta)$ et $D_{\Lambda}(\theta+1)$, relatifs à $A$ pour $\left.\theta \in\right] 0,1[$. Pour simplifier on posera

$$
\begin{array}{ll}
\|x\|_{\theta}=\|x\|_{D_{\Lambda}(\theta)}, & \text { pour } x \in D_{\Lambda}(\theta), \\
\|B\|_{\theta+1, \theta}=\|B\|_{D_{A}(\theta+1) \rightarrow D_{\Lambda}(\theta)}, & \text { pour } x \in L\left(D_{\Lambda}(\theta+1), D_{\Lambda}(\theta)\right) .
\end{array}
$$

Soit $\theta \in] 0,1\left[\right.$ fixé et soit $\varphi$ une application de $[0, T] \times D_{A}(\theta+1)$ dans $D_{A}(\theta)$, notée $t, x \rightarrow \varphi(t ; x)$. On suppose que

(i) $\varphi \in C\left([0, T] \times D_{A}(\theta+1) ; D_{A}(\theta)\right)$;

(ii) $\partial \varphi / \partial x \in C\left([0, T] \times D_{A}(\theta+1)\right) ; L\left(D_{\Lambda}(\theta+1) ; D_{A}(\theta)\right)$;

(iii) $(\partial \varphi / \partial x)(t, x)$ est la restriction à $D_{\Lambda}(\theta+1)$ d'un opérateur $\tilde{\partial \varphi} / \partial x(t, x)$ non borné dans $E$ et de domaine $D_{A}$, qui est générateur d'un semi-groupe analytique fortement continu vérifient (3.2.) dans $E$; ceci pour tout $t \in[0, T]$ et tout $x \in D_{\Lambda}(\theta+1)$

(iv) $D_{(\tilde{\partial} \tilde{\rho} / \hat{\delta} x)(t, x)}(\theta+1)=D_{A}(\theta+1)$ pout tout $t \in[0, T]$ et tout $x \in D_{A}(\theta+1)\left({ }^{10}\right)$.

THÉoRÈme 4.1. - On suppose que $\varphi$ vérifie les hypothèses (4.1.) points (i) à (iv) et que $x_{0} \in D_{A}(\theta+1)$. Alors, il existe $\tau\left(x_{0}\right)>0$ et une fonction unique

$$
u \in C\left(\left[0, \tau\left(x_{0}\right)\right] ; D_{A}(\theta+1)\right) \cap O^{1}\left(\left[0, \tau\left(x_{0}\right)\right] ; D_{\Lambda}(\theta)\right)
$$

solution de

$$
\left\{\begin{array}{l}
u^{\prime}(t)=\varphi(t, u(t)), \quad t \in[0, \tau(x)] \\
u(0)=x_{0} .
\end{array}\right.
$$

$\left[{ }^{10}\right)$ Tout ce qui va suivre reste vrai si on suppose seulement qu'il existe $\lambda:[0, T] \times$ $\times D_{A}(\theta+1) \rightarrow \boldsymbol{R}$ continu tel que

(iii) $(\widetilde{\partial \varphi} / \partial x)(t, x)-\lambda(t, x)$ soit générateur infinitésimal d'un semi-groupe analytique fortement continu, vérifiant $(3,2)$ dans $E$.

(iv) $D_{(\widetilde{\partial} \varphi / \hat{\partial} x)(i, x)-\lambda(t, x)}(\theta+1)=D_{A}(\theta+1)$,

les hypothèses (i) et (ii) restant inchangées. 
Dans la démonstration, on utilisera les quantités suivantes:

$$
L_{s, \omega}(u)(t)=\int_{s}^{t} \exp \left[(t-\sigma) \frac{\widetilde{\partial \varphi}}{\partial x}(s, x)\right] u(\sigma) d \sigma
$$

pour $s \in[0, T], x \in D_{A}(\theta+1)$. D'après le Théorème 3.1. appliqué à $A=(\widetilde{\partial \varphi} / \partial x)(s ; x)$, on sait qu'il existe $l(s ; x) \in \boldsymbol{R}$ tel que

$$
\left\|L_{s, x}\right\|_{C\left([s, T] ; D_{A}(\theta)\right) \rightarrow C\left([s, T] ; D_{A}(\theta+1)\right)} \leqslant l(s, x)
$$

et $l$ dépend continûment de $s \in[0, T]$ et $x \in D_{A}(\theta+1)$.

Ensuite on note $A_{2}$ le domaine triangulaire

$$
\{(s, t) ; t \in[0, T], s \in[0, t]\}
$$

et on pose

$$
\gamma(s, t, x, y)=\int_{s}^{t} \exp \left[(t-\sigma) \frac{\tilde{\partial \varphi}}{\partial x}(s, x)\right] \varphi(\sigma, y) d \sigma
$$

cette application est continue de $\Delta_{T} \times D_{\Lambda}(\theta+1)^{2}$ dans $D_{A}(\theta+1)$ d'après le Théorème 3.1 .

DÉmonstratron DU ThÉorème 4.1. - On pose

$$
\Psi(x)=\varphi(0, x)-y_{0}-A\left(x-x_{0}\right)
$$

où $y_{0}=\varphi\left(0, x_{0}\right)$ et $A=(\tilde{\partial \varphi} / \partial x)\left(0, x_{0}\right)$.

Il est clair que $\Psi\left(x_{0}\right)=0$ et $\Psi^{\prime}\left(x_{0}\right)=0$.

On a par conséquent

$$
\varphi(t, u(t))=A u(t)+y_{0}-A x_{0}+\{\varphi(t ; u(t))-\varphi(0, u(t))+\Psi(u(t))\}
$$

et le problème (4.2.) équivaut à l'équation intégrale

$$
u(t)=\exp [t A] x_{0}+\int_{0}^{t} \exp [(t-s) A]\left\{y_{0}-A x_{0}+\varphi(s ; u(s))-\varphi(0 ; u(s))+\Psi(u(s))\right\} d s
$$

c'est-à-dire

$$
u(t)=x_{0}+\int_{0}^{t} \exp [s A] y_{0} d s+S(u)(t)
$$

où

$$
S(u)=L_{0, x_{0}}[\varphi(\cdot, u(\cdot))-\varphi(0, u(\cdot))+\Psi(u(\cdot))]
$$


Soit $B\left(u_{0}, r\right)$ la boule de centre zéro et de rayon $r$ dans l'espace $C\left([0, \tau] ; D_{A}(\theta+1)\right)$, où $u_{0}$ désigne la fonction constante égale à $x_{0}$. On va choisir $r$ et $\tau=\tau\left(x_{0}\right)$ de manière que $S$ soit une contraction stricte dans $B\left(u_{0} ; r\right)$. Si $u$ et $v$ sont deux éléments de $B\left(u_{0}, r\right)$, on a

$$
\begin{aligned}
& \|S(u)(t)-S(v)(t)\|_{\theta+1} \leqslant l\left(0, x_{0}\right) \sup _{s \in[0, \tau]} \int_{0}^{1}\left\{\left\|\Psi^{\prime}((1-\sigma) u(s)+\sigma v(s))\right\|_{\theta+1, \theta}\right. \\
& \left.+\left\|\frac{\partial \varphi}{\partial x}(s ;(1-\sigma) u(s)+\sigma v(s))-\frac{\partial \varphi}{\partial x}(0 ;(1-\sigma) u(s)+\sigma v(s))\right\|_{\theta+1, \theta}\right\} d \sigma\|u(s)-v(s)\|_{\theta+1} \text {. }
\end{aligned}
$$

Soit

$$
r=\delta_{\partial \varphi / \partial x}\left(0, x_{0} ; \frac{1}{4 l\left(0, x_{0}\right)}\right)
$$

où $\delta_{\partial \varphi / \partial x}$ est le module de continuité de $\partial \varphi / \partial x\left({ }^{11}\right)$ au point $\left(0, x_{0}\right)$ considéré comme fonction de $D_{\Lambda}(\theta+1)$ dans $L\left(D_{A}(\theta+1) ; D_{\Lambda}(\theta)\right)$.

On a done

$$
\|S(u)-S(v)\| \leqslant \frac{1}{2}\|u-v\|
$$

dans la norme de $C\left([0, \tau] ; D_{A}(\theta+1)\right)$, pour $u$ et $v$ dans $B\left(u_{0}, r\right)$ pourvu que $\tau \leqslant r$ car il résulte de la définition de $\Psi$ que

$$
\delta_{\Psi^{\prime}}\left(x_{0} ; \varepsilon\right) \leqslant \delta_{\partial \partial / \hat{\partial} x}\left(0, x_{0} ; \varepsilon\right)
$$

pour tout $\varepsilon>0$. Le principe des contractions permet alors d'affirmer que

$$
(1-S)\left(B\left(u_{0}, r\right)\right) \supseteq B\left(u_{0}-S\left(u_{0}\right), \frac{r}{2}\right)
$$

Il en résulte que l'équation (4.5.) est résoluble dans l'espace $C\left([0, \tau] ; D_{A}(\theta+1)\right)$ à condition que

$$
\left\|u_{0}-S\left(u_{0}\right)-u_{0}-\int_{0}^{t} \exp [s A] y_{0} d s\right\| \leqslant \frac{r}{2}
$$

(11) Soient $E$ et $F$ deux espaces de Banach et soit $\Omega$ un ouvert de $E$. Si $f$ est une application continue de $\Omega$ dans $F$, le module de continuité de $f$ au point $x$ de $\Omega$ est par définition

$$
\delta_{f}(x ; \varepsilon)=\sup \left\{\delta>0, y, z \in B(x, \delta) \cap \Omega \Rightarrow\|f(x)-f(z)\|_{F} \leqslant \varepsilon\right\} \geqslant 0 .
$$

Il est clair que $\left|\delta_{f}(x ; \varepsilon)-\delta_{f}(y ; \varepsilon)\right| \leqslant\|x-y\|, \quad \forall x, y \in \Omega$ et $\forall \varepsilon>0$. 
dans la norme de $C\left([0, \tau] ; D_{A}(\theta+1)\right)$; il revient au même d'exiger que

$$
\left\|\int_{0}^{t} \exp [(t-s) A] \phi\left(s ; x_{0}\right) d s\right\|_{\theta+1}=\left\|\gamma\left(0, t, x_{0}, x_{0}\right)\right\|_{\theta+1} \leqslant \frac{r}{2} .
$$

On choisira done $\tau$ de manière que

$$
\tau \leqslant \delta_{\gamma}\left(0,0, x_{0}, x_{0} ; \frac{r}{2}\right)
$$

où $\delta_{\gamma}$ est le module de continuité de $\gamma$ au point $\left(0,0, x_{0}, x_{0}\right)$ (c.f. plus haut la note $\left.\left({ }^{11}\right)\right)$. En conclusion le Théorème 4.1. est démontré avec

$$
\left\{\begin{array}{l}
r=\delta_{\partial \varphi / \partial x}\left(0, x_{0} ; \frac{1}{4 l\left(0, x_{0}\right)}\right) \\
\tau\left(x_{0}\right)=\operatorname{Inf}\left\{\delta_{\partial \varphi / \partial x}\left(0, x_{0} ; \frac{1}{4 l\left(0, x_{0}\right)}\right) ; \delta_{\nu}\left(0,0, x_{0}, x_{0} ; \frac{r}{2}\right)\right\}
\end{array}\right.
$$

On peut montrer de surcroît que la solution $u$ dont l'existence est établie dans le Théorème 4.1. dépend continûment de la donnée $x_{0}$. Pour cela on utilisera le résultat classique qui suit:

Leman 4.2. - Soit $X$ un espace de Banach et soit pour chaque $h \in[0, H]$ une application $S_{h}$ de $B\left(z_{h} ; \varrho\right)$ dans $X$ où $B\left(z_{h} ; \varrho\right)$ désigne la boule de centre $z_{h}$ et de rayon $\varrho$ dans $X$. On suppose que $z_{h} \rightarrow z_{0}$ lorsque $h \rightarrow 0$ et que $S_{h}(z) \rightarrow S_{0}(z)$ pour tout $z \in B\left(z_{h}, \varrho\right)$ pour $h$ assez peitit. On suppose enfin qu'il existe $\alpha \in] 0,1\left[\right.$ tel que $S_{h}$ soit lipschitzienne de constante $\alpha$ dans $B\left(z_{h}, r\right)$. Alors pour $\varepsilon>0$ si $\left\|y_{h}-z_{h}+S_{h}\left(z_{h}\right)\right\| \leqslant(\varrho-\varepsilon)(1-\alpha)$ il existe $u_{h} \in B\left(z_{h} ; \varrho-\varepsilon\right)$ unique solution de

$$
u_{k}-S_{h}\left(u_{h}\right)=y_{k}
$$

et si $y_{h} \rightarrow y_{0}$ lorsque $h \rightarrow 0$, alors $u_{h} \rightarrow u_{0}$ où $u_{0} \in B\left(z_{0} ; \varrho-\varepsilon\right)$ et $u_{0}$ est solution de

$$
u_{0}-S_{0}\left(u_{0}\right)=y_{0}
$$

On en déduit la

Proposition 4.3. - On suppose que $\varphi$ vérifie les hypothèses (4.1.) (i) à (iv) et que $x_{h} \rightarrow x_{0}$ dans $D_{A}(\theta+1)$ lorsque $h \rightarrow 0$. Soit $\tau\left(x_{0}\right)$ défini en (4.7.), alors si $0<\tau_{1}<\tau\left(x_{0}\right)$, le problème

$$
\left\{\begin{array}{l}
\left.u_{h}^{\prime}(t)=p\left(t, u_{h}(t)\right), \quad t \in\right] 0, \tau_{1}[ \\
u_{h}(0)=x_{h}
\end{array}\right.
$$


admet pour $h$ assez petit, une solution unique

$$
u_{h} \in C^{1}\left(\left[0, \tau_{1}\right] ; D_{\Lambda}(\theta)\right) \cap O\left(\left[0, \tau_{1}\right] ; D_{A}(\theta+1)\right)
$$

et $u_{h} \rightarrow u$ dans $O\left(\left[0, \tau_{1}\right] ; D_{A}(\theta+1)\right)$ où $u$ est solution de (4.2.).

DÉmonstration. - En suivant le même chemin que dans la démonstration du Théorème 4.1. on voit que le problème (4.8.) équivaut à l'équation intégrale

$$
u_{h}(t)=x_{h}+\int_{0}^{t} \exp \left[s \frac{\tilde{\partial \varphi}}{\partial x}\left(0, x_{h}\right)\right] \varphi\left(0, x_{h}\right) d s+S_{h}\left(u_{h}\right)(t)
$$

où

$$
S_{h}(u)(t)=L_{0, x_{h}}\left[\varphi(\cdot, u(\cdot))-\varphi(0, u(\cdot))+\Psi_{h}(u(\cdot))\right]
$$

et

$$
\Psi_{h}(x)=\varphi(0, x)-\varphi\left(0, x_{h}\right)-\frac{\widetilde{\partial \varphi}}{\partial x}\left(0, x_{h}\right) \cdot\left(x-x_{h}\right)
$$

Ensuite on applique le Lemme 4.2. avec pour espace $X$ l'espace $C\left(\left[0, \tau_{1}\right] ; D_{\Lambda}(\theta+1)\right)$, avec l'application $S_{h}$ définie ci-dessus, $z_{h}$ désignant la fonction constante égale à $x_{h}$, $\varrho<r$ défini par (4.6.) et $\alpha=\frac{1}{2}$. Enfin $y_{h}$ désigne la fonction

$$
t \mapsto x_{h}+\int_{0}^{t} \exp \left[s \frac{\widetilde{\partial \varphi}}{\partial x}\left(0, x_{h}\right)\right] \varphi\left(0, x_{k}\right) d s
$$

L'application $S_{h}$ est définie dans $X$ entier, mais en répétant les calculs de la démonstration $d u$ Théorème 4.1., on voit que $S_{h}$ est lipschitzienne de constante $\frac{1}{2}$ dans $B\left(z_{h} ; r_{h}\right)$ pourvu que $\tau_{1} \leqslant r_{h}$ et que

$$
r_{h}=\delta_{\partial \varphi / \partial x}\left(0, x_{h}, \frac{1}{4 l\left(0, x_{h}\right)}\right)
$$

Il est clair que $r_{h} \rightarrow r$ grâce à la continuité de $l$ et $\delta_{\partial \varphi / \hat{w} w}$, done la condition $\tau_{\mathbf{1}}<r$ implique $\tau_{1} \leqslant r_{h}$ et la condition $\varrho<r$ implique $\delta \leqslant r_{h}$ pour $h$ assez petit. Les hypothèses $d u$ Lemme 4.2. concernant $S_{h}$ sont donc vérifiées.

Ensuite la condition $\left\|y_{h}-z_{h}+S_{k}\left(z_{h}\right)\right\| \leqslant(\varrho-\varepsilon)(1-\alpha)$ signifie ici que

$$
\left\|\int_{0}^{t} \exp \left[(t-s) \frac{\tilde{\partial \varphi}}{\partial x}\left(0, x_{h}\right)\right] \varphi\left(s ; x_{h}\right) d s\right\|_{\theta+1} \leqslant \frac{\varrho-\varepsilon}{2}
$$


pour $t \in\left[0, \tau_{1}\right]$; il revient au même de demander que

$$
\left\|\gamma\left(0, t, x_{h}, x_{h}\right)\right\|_{\theta+1} \leqslant \frac{o-\varepsilon}{2}
$$

ce qui est réalisé dès que

$$
\tau_{1} \leqslant \delta_{\nu}\left(0,0, x_{h} ; \frac{\varrho-\varepsilon}{2}\right)
$$

Par hypothèse on a $\tau_{1}<\tau\left(x_{0}\right) \leqslant \delta_{\gamma}\left(0,0, x_{0}, x_{0} ; r / 2\right)$ et $\delta_{y}$ est une fonction continue de tous ses arguments donc en choisissant $\varrho$ assez proche de $r, \varepsilon$ assez petit, la condition (4.10.) est réalisée pour $h$ assez petit. Ceci montre que la propriété 4.3. résulte du Lemme 4.2.

REMARQUE 4.4. - Quelques modifications mineures des démonstrations précédentes permettent d'obtenir également les résultats du Théorème 4.1. et de la Proposition 4.3. sous l'hypothèse plus faible qui suit où $\Omega$ esţ un ouvert de $D_{A}(\theta+1)$ :

(i) $\varphi \in C\left([0, T] \times \Omega ; D_{A}(\theta)\right)$;

(ii) $\partial \varphi / \partial x \in C\left([0, T] \times \Omega ; L\left(D_{\Lambda}(\theta+1), D_{\Lambda}(\theta)\right)\right)$;

(iii) $(\partial \varphi / \partial x)(t, x)$ est la restriction à $D_{A}(\theta+1)$ d'un opérateur $(\widetilde{\partial \varphi} / \partial x)(t, x)$ non borné dans $E$, de domaine $D_{A}$, qui est générateur infinitésimal d'un semigroupe analytique fortement continu dans $E$, pour tout $t \in[0, T]$ et tout $x \in \Omega$;

(iv) $D_{(\tilde{\partial} \varphi / \hat{x} x)(t, x)}(\theta+1)=D_{A}(\theta+1)$ pour tout $t \in[0, T]$ et tout $x \in \Omega$.

REMarque 4.5. - On considère les problèmes

$$
\left\{\begin{array}{l}
u_{h}^{\prime}(t)=\varphi\left(t, u_{h}(t)\right) \\
u_{h}\left(t_{0}\right)=x_{h}
\end{array}\right.
$$

pour $h \in\left[0, h_{0}\right]$ avec $x_{h} \rightarrow x_{0}$ dans $D_{A}(\theta+1)$ où $\varphi$ vérifie (4.1.).

On pose

$$
\varrho\left(t_{0}, x_{0}\right)=\frac{1}{2} \operatorname{Inf}\left\{r, \delta_{\gamma}\left(t_{0}, t_{0}, x_{0}, x_{0}, \frac{r}{2}\right), T-t_{0}\right\}
$$

avec

$$
r=\delta_{\partial \varphi / \partial x}\left(0, x_{0}, \frac{1}{4 l\left(t_{0}, x_{0}\right)}\right)
$$

conformément à $(4,7$.$) . Alors d'après la Proposition 4.3. les Problèmes (4.11.) ont$ une solution unique continue à valeurs dans $D_{A}(\theta+1)$ dans l'intervalle $\left[t_{0}, t_{0}+\varrho\left(t_{0}, x_{0}\right)\right]$ et $u_{h} \rightarrow u_{0}$ lorsque $h \rightarrow 0$ dans la norme de $C\left(\left[t_{0}, t_{0}+\delta\left(t_{0}, x_{0}\right)\right] ; D_{\Lambda}(\theta+1)\right)$. 
4.2. On continue ici l'étude de l'équation (4.2.) dans l'espoir cette fois de la résoudre globalement. Classiquement on utilise la notion de solution maximale: on dit que $u \in O\left([0, \tau) ; D_{A}(\theta+1)\right)$ est maximale si elle ne peut pas être prolongée à un intervalle plus grand en tant que solution de (4.2.) (on considère ici soit des intervalles fermés à droite, soit des intervalles ouverts à droite). Grâce au Lemme de Zorn, pour tout $x_{0} \in D_{\Lambda}(\theta+1$ ) (ou $\Omega$ dans l'hypothèse de. la Remarque 4.4.), il existe une solution maximale $u_{x_{0}}$ définie dans l'intervalle $I_{x_{0}}$ continue à valeurs dans $D_{A}(\theta+1)$.

Cette solution a les propriétés suivantes:

Proposition 4.6. - Sous les hypothèses du Théorème 4.1. on a

(i) $\int I_{x_{0}}$ est ouvert dans $[0, T]$;

(ii) $\left\{u_{x_{0}}\right.$ est unique.

DÉmonstration. - S'agissant de solutions classiques on peut utiliser les raisonnements habituels:

(i) Pour tout $t_{0} \in I_{x_{0}}$ avec $t_{0}<T$, le problème

$$
\left\{\begin{array}{l}
v^{\prime}(t)=\varphi(t, v(t)) \\
v\left(t_{0}\right)=u_{x_{0}}\left(t_{0}\right)
\end{array}\right.
$$

admet d'après la Remarque 4.5. une solution unique dans $\left[t_{0}, t_{0}+\varrho\left(t_{0}, u_{x_{0}}\left(t_{0}\right)\right)\right]$ qui prolonge $u_{x_{0}}$; done $I_{x_{0}} \supset\left[t_{0}, t_{0}+\varrho\left(t_{0}, u_{\dot{x}_{0}}\left(t_{0}\right)\right)\right]$ ce qui prouve que $I_{x_{0}}$ est ouvert.

(ii) Soient $u$ et $v$ deux solutions du problème $(4.2$.) dans $[0, \varrho]$, alors grâce au Théorème 4.1. on a $u=v$ dans $[0, \eta]$ avec $\eta>0$. On introduit alors

$$
\tau=\operatorname{Max}\{t \in[0, \varrho] ; u(s)=v(s) \text { pour } s \in[0, t]\} ;
$$

si $\tau<\varrho$ on considère le problème

$$
\left\{\begin{array}{l}
v^{\prime}(t)=\varphi(t, v(t)) \\
v(\tau)=u(\tau)
\end{array}\right.
$$

et il résulte $\mathrm{du}$ Théorème 4.1. que $u=v$ dans $\left[\tau, \tau^{\prime}\right]$ avec $\tau^{\prime}>\tau$ ce qui contredit la définition de $\tau$. En conséquence, on a $\tau=\varrho$.

Dans la suite on notera $\sigma\left(x_{0}\right)$ l'extrémité de $I_{x_{0}}$ c'est-à-dire que

$$
I_{x_{0}}=\left[0, \sigma\left(x_{0}\right)\left[\quad \text { si } \quad \sigma\left(x_{0}\right)<T .\right.\right.
$$

On posera également

$$
\Gamma=\left\{(t, x) \in[0, T] \times D_{A}(\theta+1) ; t \in I_{x}\right\} u(t, x)=u_{x}(t) .
$$


Giuseppe Da Prato - Pierire Grisvard: Equations d'évolution abstraites, etc.

Proposition 4.7. - Sous les hypothèses du Théorème 4.1., $\Gamma$ est ouvert dans $[0, T] \times$ $\times D_{A}(\theta+1)$ et $u: \Gamma \rightarrow D_{\Lambda}(\theta+1)$ est continu.

Démonstration. - Soit $x_{h} \rightarrow x_{0}$ dans $D_{A}(\theta+1)$ lorsque $h \unlhd 0$ et soit $\sigma^{\prime}<\sigma\left(x_{0}\right)$; on va démontrer que

(i) $\sigma\left(x_{h}\right)>\sigma^{\prime}$ pour $h$ assez petit;

(ii) $u_{x_{h}} \rightarrow u_{x_{0}}$ dans $C\left(\left[0, \sigma^{\prime}\right] ; D_{A}(\theta+1)\right)$ ce qui prouvera la proposition.

Puisque $\sigma^{\prime}<\sigma\left(x_{0}\right)$ on a $\varrho(t, u(t))>0$ pour tout $t \in\left[0, \sigma^{\prime}\right]$ où $\varrho$ est défini par (4.12.). Comme de plus $\varrho$ est continue toujours d'après (4.12.) on a

$$
\varepsilon=\operatorname{Min}\left\{\varrho(t, u(t)), t \in\left[0, \sigma^{\prime}\right]\right\}>0 \text {. }
$$

Il suffit alors d'appliquer la Proposition 4.3. dans chacun des intervalles d'un partage de $\left[0, \sigma^{\prime}\right]$ en intervalles de longueur au plus égale à $\varepsilon$, pour conclure. C.Q.F.D.

\section{5. - Equations non linéaires, existence globale.}

5.1. Concernant les résultats obtenus au $\$ 4$ on peut observer ce qui suit: si $\varrho(t, x)$ défini en (4.12.) a une borne inférieure strictement positive lorsque $(t, x)$ varie dans $[0, T] \times D_{A}(\theta+1)$, alors on a toujours $\sigma(x)=T$. Cependant il est facile de voir que cela entraîne que $\gamma$ est uniformément continu done que $D_{A}=E$.

Cette condition n'est pas réalisée dans la plupart des applications en vue; par conséquent il faudra utiliser d'autres méthodes pour obtenir $\sigma(x)=T$ e'est-à-dire l'existence globale.

Une méthode possible consiste à utiliser des inégalités à priori; voici un résultat dans ce sens:

Proposition 5.1. - On suppose que $\varphi$ vérifie l'hypothèse (4.1.) pour tout $\theta \in\left[\theta_{1}, \theta_{2}\right]$ $\left(0<\theta_{1}<\theta_{2}<1\right)$. On suppose de plus que pour tout borné $B$ de $D_{\Delta}\left(\theta_{2}+1\right)$, l'ensemble $\varphi([0, T] \times B)$ est borné dans $D_{A}\left(\theta_{2}\right)$. Soit enfin $x_{0} \in D_{A}\left(\theta_{2}+1\right)$ tel que la solution maximale $u_{x_{0}}$ du problème (4.2.) dans l'espace $D_{A}\left(\theta_{1}+1\right)$ prenne ses valeurs dans $D_{A}\left(\theta_{2}+1\right)$ et vérifie:

$$
\left\|u_{x_{0}}(t)\right\|_{\theta_{2}+1} \leqslant M
$$

pour tout $t \in I_{x_{0}}$. Alors $\sigma\left(\hat{x}_{0}\right)=T$.

DÉmonstration. - On observe d'abord que $u_{x_{0}}$ est continue dans $I_{x_{0}}$ à valeurs dans $D_{A}(\theta+1)$ pour $\theta \in\left[\theta_{1}, \theta_{2}[\right.$.

En effet d'après le Théorème 2.15. on a pour $\theta_{1}<\theta<\theta_{2}$ et $t, s \in I_{x_{0}}$, l'inégalité

$$
\left\|u_{x_{0}}(t)-u_{x_{0}}(s)\right\|_{\theta+1} \leqslant C \quad\left\|u_{x_{0}}(t)-u_{x_{\theta}}(s)\right\|_{\theta_{1}+1}^{1-\alpha}\left\|u_{x_{0}}(t)-u_{x_{0}}(s)\right\|_{\theta_{2}+1}^{\alpha}
$$


où $\alpha$ est tel que $\theta=(1-\alpha) \theta_{1}+\alpha \theta_{2}$. Tenant compte de (5.1.) on a donc $\| u_{x_{0}}(t)-$ $-u_{x_{0}}(s)\left\|_{\theta+1} \leqslant C(2 M)^{\alpha}\right\| u_{x_{0}}(t)-u_{x_{0}}(s) \|_{\theta_{1}+1}^{1-\alpha}$ et la continuité de $u_{x_{0}}$ à valeurs dans $D_{A}\left(\theta_{0}+1\right)^{\theta_{1}+1}$ implique que $\left\|u_{x_{0}}(t)-u_{x_{0}}(s)\right\|_{\theta+1} \rightarrow 0$ lorsque $t \rightarrow s$ dans $I_{x_{0}}$.

Ceci étant on réécrit l'équation (4.2.) sous la forme

$$
\left\{\begin{array}{l}
u_{x_{0}}^{\prime}(t)=\Lambda u_{x_{0}}(t)+\varphi\left(t, u_{x_{0}}(t)\right)-A u_{x_{0}}(t) \\
u_{x_{0}}(0)=x_{0}
\end{array}\right.
$$

pour $t \in I_{x_{i}}$. La fonction

$$
f(t)=\varphi\left(t, u_{x_{0}}(t)\right)-A u_{x_{0}}(t)
$$

est bornée à valeurs dans $D_{A}\left(\theta_{2}\right)$ done à fortiori dans $D_{A}(\theta)$ où $\theta_{1}<\theta<\theta_{2}$; de plus d'après ce qui précède elle est continue done mesurable. En somme on a

$$
f \in L^{\infty}\left(\left[0, \sigma\left(x_{0}\right)\left[; D_{A}(\theta)\right)\right.\right.
$$

et on déduit de la Proposition 3.4. que

$$
u_{x_{6}} \in C\left(\left[0, \sigma\left(x_{0}\right)\right] ; D_{\Lambda}\left(\theta_{1}+1\right)\right)
$$

Cependant comme $u_{x_{0}}$ est la solution maximale dans $D_{A}\left(\theta_{1}+1\right)$ ceci n'est possible que si $\sigma\left(x_{0}\right)=T$. C.Q.F.D.

Parmi les hypothèses de la Proposition 5.1., figure une propriété de régularité de la solution correspondant à une donnée initiale plus régulière. On va donner ici des hypothèses sur $\varphi$ permettant d'obtenir ce type de régularité.

THÉoRène 5.2. - On suppose que $\varphi$ vérifie les hypothèses (4.1.) pour tout $\theta=\theta_{0}$, $\theta_{1}$ et $\theta_{1}+1\left(0<\theta_{0}<\theta_{1}<1\right)$. On suppose de plus que

$$
\phi \in C^{1}\left([0, T] \times D_{A}\left(\theta_{1}+1\right) ; D_{A}\left(\theta_{1}\right)\right)
$$

et que $x \mapsto \varphi(t, x)$ est pour tout $t$ un homéomorphisme de $D_{A}\left(\theta_{1}+2\right)$ sur $D_{A}\left(\theta_{1}+1\right)$ dont l'inverse dépend continûment de t.

Soit $x_{0} \in D_{A}\left(\theta_{1}+2\right)$ et $u_{x_{0}}$ la solution maximale du problème (4.2.) dans l'espace $D_{A}\left(\theta_{1}+1\right)$, alors $u_{x_{9}}$ est continue à valeurs dans $D_{A}\left(\theta_{1}+2\right)$.

Démonstration. - Soit $I_{x_{0}}=\left[0, \tau\left[\right.\right.$ l'intervalle de définition de $u=u_{x_{0}}$. L'idée est de prouver que la dérivée de $u$ par rapport à $t$ est la solution du problème

$$
\left\{\begin{array}{l}
v_{0}^{\prime}(t)=\frac{\partial \varphi}{\partial x}(t, u(t)) v_{0}(t)+\frac{\partial \varphi}{\partial t}(t, u(t)) \\
v_{0}(0)=\varphi\left(0, x_{0}\right)
\end{array}\right.
$$


Dans ce problème qui est linéaire les données sont $\varphi\left(0, x_{0}\right) \in D_{\Lambda}\left(\theta_{1}+1\right)$ et la fonction $t \rightarrow(\partial \varphi / \partial t)(t, u(t))$ qui est continue dans $\left[0, \tau\left[\grave{a}\right.\right.$ valeurs dans $D_{\Lambda}\left(\theta_{1}\right)$. De plus la famille des opérateurs

$$
A_{0}(t)=\frac{\partial \varphi}{\partial x}(t, u(t))
$$

vérifie pour $t \in[0, T], T<\tau$, les hypothèses du Théorème 3.6. à condition de remplacer $E$ par $D_{A}\left(\theta_{0}\right)$ et $\theta$ par $\theta_{1}-\theta_{0}$ (en tenant compte du Théorème 2.15.) (12). L'application du Théorème 3.6. montre alors que $v_{0} \in C\left([0, T] ; D_{A}\left(\theta_{1}+1\right)\right)$; compte tenu de l'inversibilité de $\varphi$ on en déduit que $u \in C\left([0, T] ; D_{A}\left(\theta_{1}+2\right)\right)$.

Cependant, il n'est pas possible d'obtenir directement (5.2.) par dérivation de (4.2.) ear $(\partial \varphi / \partial u)(t, u(t))$ ne peut pas, à priori, être calculé sur $u^{\prime}(t) \in D_{4}\left(\theta_{1}\right)$. C'est pourquoi on utilise une méthode où on pose

$$
v_{h}(t)=\frac{u(t+h)-u(t)}{h}, \quad 0 \leqslant t \leqslant \tau-h
$$

avec $0<h<\tau$. Il est clair que $v_{h}$ est la solution du problème linéaire suivant

$$
\left\{\begin{array}{l}
v_{h}^{\prime}(t)=A_{h}(t) v_{h}(t)+f_{h}(t), \quad t \in[0, \tau-h[ \\
v_{h}(0)=\xi_{h}
\end{array}\right.
$$

où

$$
\begin{aligned}
& f_{h}(t)=\frac{1}{h} \int_{0}^{h} \frac{\partial \varphi}{\partial t}(t+s ; u(t+h)) d s \\
& A_{h}(t)=\int_{0} \frac{\partial \varphi}{\partial x}(t, u(t)+s[u(t+h)-u(t)]) d s \quad \xi_{h}=\frac{u(h)-x}{h} .
\end{aligned}
$$

Il est clair également que $f_{h} \in C\left(\left[0, \tau-h\left[; D_{\Lambda}\left(\theta_{1}\right)\right)\right.\right.$ et que

$$
A_{h} \in C\left(\left[0, \tau-h\left[; L\left(D_{\Lambda}\left(\theta_{1}+1\right) ; D_{A}\left(\theta_{1}\right)\right)\right) .\right.\right.
$$

Enfin, si on a choisi $h$ assez petit pour qu'il soit dans l'intervalle d'existence de la solution maximale de (4.2.) dans $D_{A}\left(\theta_{1}+2\right)$ (application du Théorème 4.1. avec $\left.\theta=\theta_{1}+1\right)$ alors lorsque $h \rightarrow 0$ on a

$$
\xi_{h} \rightarrow u^{\prime}(0)=\varphi\left(0, x_{0}\right)=\xi_{0}
$$

${ }^{\left({ }^{2}\right)}$ On aurait pu éviter cette complication en faisant l'hypothèse supplémentaire que

$$
\frac{\widetilde{\partial \varphi}}{\partial x} \in C\left([0, T] \times D_{\Lambda}(\theta+1) ; L\left(D_{\Lambda} ; E\right)\right) \text {. }
$$


dans $D_{A}\left(\theta_{1}+1\right)$ (et non $D_{A}\left(\theta_{1}\right)$ comme on pourrait s'y attendre à première vue). Il est clair de plus que $f_{k}(t) \rightarrow f_{0}(t)=(\partial \varphi / \partial t)(t, u(t))$ dans $D_{A}\left(\theta_{1}\right)$ uniformément dans $[0, T]$ pour tout $t<\tau$ lorsque $h \rightarrow 0$.

On va maintenant montrer que $v_{h} \rightarrow v_{0}$ solution de (5.2.) dans $D_{A}\left(\theta_{1}+1\right)$, uniformément sur tout compact de $\left[0, \tau\left[\right.\right.$. Précisément, on suppose savoir que $v_{h} \rightarrow v_{0}$ dans $D_{A}\left(\theta_{1}+1\right)$ uniformément dans $[0, \beta]$, ce qui est vrai au moins arec $\beta=0$ et on va montrer qu'il existe $\sigma>0$ indépendant de $\beta$ tel que $v_{h} \rightarrow v_{0}$ dans $D_{A}\left(\theta_{1}+1\right)$ uniformément dans $[\beta, \beta+\sigma]$. Pour cela on pose pour $h \geqslant 0$

$$
w_{h}(t)=v_{h}^{t}(t)-A_{0}(\beta) v_{h}(t) .
$$

On a alors

$$
v_{h}(t)=\exp \left[(t-\beta) A_{0}(\beta)\right] v_{h}(\beta)+\int_{\beta}^{t} \exp \left[(t-s) A_{0}(\beta)\right] w_{h}(s) d s .
$$

D'autre part, il est clair que

$$
w_{h}(t)=\left[A_{h}(t)-A_{0}(\beta)\right] v_{h}(t)+f_{h}(t)
$$

on en déduit l'équation suivante qui définit $w_{h}$

$$
\begin{aligned}
& w_{h}(t)=\left[A_{h}(t)-A_{0}(\beta)\right] \exp \left[(t-\beta) A_{0}(\beta)\right] v_{h}(\beta)+f_{h}(t)+ \\
& +\left[A_{h}(t)-A_{0}(\beta)\right] \int_{\beta}^{t} \exp \left[(t-s) A_{0}(\beta)\right] w_{h}(s) d s .
\end{aligned}
$$

On va prouver que $w_{h}$ converge vers $w_{0}$ en appliquant le Lemme 4.2. de la manière suivante: $X=C\left([\beta, \beta+\sigma] ; D_{\Lambda}\left(\theta_{1}\right)\right), z_{h}=0, \varrho=+\infty$,

et $y_{h}$ est la fonction

$$
s_{h}(w)(t)=\left[A_{h}(t)-A_{0}(\beta)\right] \int_{\beta}^{t} \exp \left[(t-s) A_{0}(\beta)\right] w(s) d s
$$

$$
t \rightarrow\left[A_{h}(t)-A_{0}(\beta)\right] \exp \left[(t-\beta) A_{0}(\beta)\right] v_{h}(\beta)+f_{h}(t) .
$$

Si on a fixé $T<\tau$ et si on suppose $\beta+\sigma \leqslant T$, il résulte de la définition de $A_{h}$ que $A_{h}(t) \rightarrow A_{0}(\beta)$ lorsque $h \rightarrow 0$ et $t \rightarrow \beta$, uniformément pour $\beta \in[0, T]$ et ceci dans la norme de

$$
L\left(D_{\Lambda}\left(\theta_{1}+1\right), D_{\Lambda}\left(\theta_{1}\right)\right){ }^{(13)}
$$

(13) En effet, on utilise $(\partial p / \partial x)(t, x)$ pour $x \in R$ le compact de $D_{A}\left(\theta_{1}+1\right)$ image de $[0, T] \times[0,1] \times[0, H)(H$ assez petit) par $t, s, h \mapsto u(t)+s[u(t+h)-u(t)]$. 
D'autre part les $A_{0}(\beta)$ sont uniformément générateurs infinitésimaux de semi-groupes analytiques (voir le point 3.2.) pour $\beta \in[0, T]$.

On en déduit que les hypothèses đu Lemme 4.2. sont vérifiées avee une constante de Lipschitz $\alpha$ indépendante de $\beta$ pourvu que $\sigma$ soit assez petit. Le Lemme 4.2. montre que $w_{h} \rightarrow w_{0}$ dans $D_{A}\left(\theta_{1}\right)$ uniformément dans $[\beta, \beta+\sigma]$. Ensuite en résolvant

$$
v_{h}^{\prime}(t)=A_{0}(\beta) v_{h}(t)+w_{h}(t)
$$

on voit que $v_{h}(\beta) \rightarrow v_{0}(\beta)$ dans $D_{A}\left(\theta_{1}+1\right)$ implique que $v_{h} \rightarrow v_{0}$ dans $D_{A}\left(\theta_{1}+1\right)$ uniformément dans $[\beta, \beta+\sigma]$. On en déduit done que $v_{h} \rightarrow v_{0}$ dans $D_{A}\left(\theta_{1}+1\right)$, uniformément dans $[0, T]$ pour tout $T<\tau$. En conclusion on a done $v_{0}=u^{\prime}$ et le résultat en découle suivant le raisonnement annoncé en début de démonstration.

Remaroue 5.3. - Si on réunit les hypothèses de la Proposition 5.1. et du Théorème 5.2. on obtient pour $\theta \in\left[\theta_{0}, \theta_{2}\right] \cup\left\{\theta_{2}+1\right\}, 0<\theta_{0}<\theta_{2}<1$

(i) $\varphi \in C^{1}\left([0, T] \times D_{A}(\theta+1) ; D_{A}(\theta)\right)$;

(ii) $(\partial \varphi / \partial x)(l, x)$ est la restriotion à $D_{A}(\theta+1)$ d'un opérateur $(\widetilde{\partial \varphi} / \partial x)(t, x)$ de domaine $D_{A}$ dans $E$, qui est générateur d'un semingroupe analytique fortement continu dans $E$ pour tout $(t, x) \in[0, T] \times D_{A}(\theta+1)$;

(iii) $D_{(\tilde{\rho} \tilde{\partial} / \partial x)\langle t, x)}(\theta+1)=D_{\Lambda}(\theta+1)$ pour tout $(t, x) \in[0, T] \times D_{\Lambda}(\theta+1)$;

(iv) $\varphi([0, T] \times B)$ est borné dans $D_{A}\left(\theta_{1}\right)$ pour tout borné $B$ de $D_{A}\left(\theta_{1}+1\right)$ pour un $\left.\theta_{1} \in\right] \theta_{0}, \theta_{2}[$

(v) $x \rightarrow \varphi(t, x)$ est pour tout $t \in[0, T]$ un homéomorphisme de $D_{A}\left(\theta_{1}+2\right)$ sur $D_{A}\left(\theta_{1}+1\right)$, dont l'inverse dépend contimûment de $t$.

Sous ces hypothèses $x_{0} \in D_{A}\left(\theta_{1}+2\right)$ et soit $u_{x_{0}} \in C\left(\left[0, \tau_{x_{0}}\left[D_{A}\left(\theta_{0}+1\right)\right)\right.\right.$ la solution maximale de (4.2.) dans $D_{A}\left(\theta_{1}+1\right)$. On suppose qu'il existe $M$ tel que

$$
\left\|u_{x_{6}}(t)\right\|_{\theta_{2}+1} \leqslant M
$$

pout tout $t \in I_{x_{0}} ;$ alors $\tau_{x_{0}}=T$ et $u_{x_{0}} \in C\left([0, T] ; D_{A}\left(\theta_{1}+2\right)\right)$.

\section{6. - Espaces de Nikolski.}

6.1. Dans les exemples de problèmes mixtes examinés au $\S 7$ on est amené à expliciter les espaces $D_{A}(\theta)$ lorsque $X=L_{y}(\Omega)$ où $\Omega$ est un ouvert borné assez régulier de $\boldsymbol{R}^{n}$ et $D_{A}$ est un sous-espace de $W_{n}^{m}(\Omega)$ défini par certaines conditions aux limites homogènes de sorte que

$$
\stackrel{\circ}{W}_{D}^{m}(\Omega) \subseteq D_{A} \subseteq W_{p}^{m}(\Omega)
$$

où $\stackrel{0}{W}_{p}^{m}(\Omega)$ désigne la fermeture de $\mathscr{D}(\Omega)$ dans $W_{p}^{m}(\Omega)$. 
Gruseppe Da Prato - Pierre Grisvard: Equations d'évolution abstraites, ete. 367

Pour la suite il est commode d'introduire un nouvel espace de Nikolski (c.f. les exemples 2.9. et 2.11.):

Définition 6.1. - Pour $1<p<+\infty$ et $s$ réel $>0$ non entier $(s=k+\sigma, k$ entier, $0<\sigma<1)$ on désigne par $h_{p}^{(s)}\left(\boldsymbol{R}^{n}\right)$ le sous-espace de $W_{p}^{k}\left(\boldsymbol{R}^{n}\right)$ défini par les conditions

$$
t^{-\sigma}\left(\int_{\boldsymbol{R}^{n}}\left[D^{\alpha} u\left(x+t e_{j}\right)-\left.D^{\alpha} u(x)\right|^{p} d x\right)^{1 / p} \rightarrow 0\right.
$$

pour tout multientier $\alpha$ de longueur $k$ et $j=1,2, \ldots, n$.

Si $\Omega$ est un ouvert de $\boldsymbol{R}^{n}$, on désigne par $h_{p}^{(s)}(\Omega)$ l'espace des restrictions à $\Omega$ des éléments de $h_{p}^{(s)}\left(\boldsymbol{R}^{n}\right)$.

De l'exemple 2.11. et du théorème de prolongement simultané des espaces de Sobolev de STErr [22], on déduit la

Proposition 6.2. - Si $\Omega$ est borné et à frontière Lipsohitzienne, on a $h_{p}^{s}(\Omega)=$ $=\left(W_{p}^{m}(\Omega) ; L_{p}(\Omega)\right)_{\theta}$ si $s=m(1-\theta)$ est non entier.

L'espace $h_{p}^{s}(\Omega)$ peut aussi être caractérisé de manière explicite comme suit.

On considère d'abord le cas où $0<8<1$ et $\Omega=\boldsymbol{R}_{+}^{n}=\left\{x \in \boldsymbol{R}^{n} ; x_{n}>0\right\}$; les Théorèmes 2.6. et 2.8. appliqués à $A_{j}=\partial / \partial x_{j}$ permettent d'affirmer que $u \in h_{p}^{s}\left(\boldsymbol{R}_{+}^{n}\right)$ si $u \in L_{p}\left(\boldsymbol{R}_{+}^{n}\right)$

$$
t^{-s}\left(\int_{\boldsymbol{R}_{+}^{n}}\left|u\left(x+t e_{j}\right)-u(x)\right|^{p}\right)^{1 / p} \rightarrow 0
$$

lorsque $t \searrow 0$ pour $j=1,2, \ldots, n$. Il revient au même d'exiger que

$$
\|y\|^{-s}\left(\int_{\boldsymbol{R}_{+}^{n}\left\{\left\{\boldsymbol{R}_{+}^{n}-y\right\}\right.}|u(x+y)-u(x)|^{p} d x\right)^{1 / p} \rightarrow 0,
$$

lorsque $y \rightarrow 0$ dans $\boldsymbol{R}^{n}$. Par des changements de coordonnées locales bilipschitziens on en déduit que si $\Omega$ est borné et à frontière lipschitzienne alors $u \in h_{y}^{g}(\Omega)$ si $u \in L_{p}(\Omega)$ et

$$
\|y\|^{-s}\left(\int_{\Omega \cap\{\Omega-y\}}|u(x+y)-u(x)|^{p} d x\right)^{1 / p} \rightarrow 0
$$

lorsque $y \rightarrow 0$ dans $\boldsymbol{R}^{n}$.

Ensuite pour $s$ général on remarque que grâce aux énoncés 2.14. et 2.15. appliqués à $A=(1-4)^{\frac{1}{2}}$ et grâce au Théorème de MrHurn [17], on a l'identité

$$
h_{p}^{s}(\Omega)=\left(W_{p}^{k+1}(\Omega) ; W_{p}^{k}(\Omega)\right)_{\sigma}
$$


et par conséquent c'est le sous-espace de $W_{p}^{k}(\Omega)$ défini par la condition $D^{\alpha} u \in h_{p}^{\sigma}(\Omega)$ pour tout $\alpha$ de longueur $k$.

On va maintenant reprendre cette étude en remplaçant $W_{p}^{m}(\Omega)$ par $\stackrel{\circ}{W}_{p}^{m}(\Omega)$ en supposant pour simplifier, que $\Omega$ est borné et à frontière de classe $C^{m}$. Il est alors bien connu que e'est le sous-espace de $W_{y}^{m}(\Omega)$ défini par l'annulation de $u$ et de ses dérivées jusqu'à l'ordre $m-1$ sur $\Gamma$ (frontière de $\Omega$ ).

On va d'abord élucider le cas particulier où $m(1-\theta)=v$ avee $0<\sigma<1 / p$. On a alors le

THÉOR亡̀ne 6.3. - Si $\Omega$ est borné et à frontière de classe $C^{m}$, on a $h_{p}^{\sigma}(\Omega)=\left(\stackrel{\circ}{W}_{p}^{m}(\Omega)\right.$; $\left.L_{p}(\Omega)\right)_{0}$ si $\sigma=(1-\theta)<1 / p$.

Ceci montre que pour $\sigma=m(1-\theta)$ assez petit les espaces $\left(W_{p}^{\text {in }}(\Omega) ; L_{p}(\Omega)\right)_{\theta}$ et $\left(\mathfrak{W}_{p}^{m}(\Omega) ; L_{p}(\Omega)\right)_{\theta}$ coincident. En particulier on en déduit que

$$
D_{A}(\sigma / m)=h_{p}^{\sigma}(\Omega)
$$

pour $\sigma<1 / p$, chaque fois que

$$
\hat{W}_{p}^{m}(\Omega) \subseteq D_{A} \subseteq W_{p}^{m}(\Omega)
$$

algébriquement et topologiquement; ce sera la situation la plus fréquente. Malheureusement l'identité du Théorème 6.3. n'est plus vraie lorsque $\sigma \geqslant 1 / p$ comme on le verra plus loin.

L'énoncé du Théorème 6.3. se déduit du cas où $\Omega=\boldsymbol{R}_{+}^{n}$ par cartes locales.

Démonstration. - 1ère étape. On démontre d'abord que

$$
\left(\stackrel{\circ}{W}_{g}^{m}\left(\boldsymbol{R}_{+}^{n}\right) ; L_{p}\left(\boldsymbol{R}_{+}^{n}\right)\right)_{\theta}=\left(\stackrel{\circ}{W_{p}^{1}}\left(\boldsymbol{R}_{+}^{n}\right) ; L_{p}\left(\boldsymbol{R}_{+}^{n}\right)\right)_{\sigma} .
$$

En effet, en donnant un rôle privilégié à la variable $x_{n}$, on peut écrire (grâce au Théorème de MrHurn [17]) que

$$
\stackrel{\circ}{W}_{y}^{m}\left(\boldsymbol{R}_{+}^{n}\right)=\stackrel{\circ}{W}_{y}^{m}\left(\boldsymbol{R}_{+} ; L_{p}\left(\boldsymbol{R}^{n-1}\right)\right) \cap L_{p}\left(\boldsymbol{R}_{+} ; W_{p}^{m}\left(\boldsymbol{R}^{n-1}\right)\right)
$$

et par conséquent grâce au Lemme 2.7. appliqué avec

$$
X=L_{p}\left(\boldsymbol{R}_{+}^{n}\right), \quad Z=\dot{\mathscr{W}}_{p}^{m}\left(\boldsymbol{R}_{+} ; L\left(\boldsymbol{R}^{n-1}\right)\right) \quad \text { et } \quad A=\left[1-\sum_{j=1}^{n-1}\left(\frac{\partial}{\partial x_{j}}\right)^{2}\right]^{m / 2}
$$

on voit que $\left(\stackrel{\circ}{W}_{p}^{m}\left(\boldsymbol{R}_{+}\right) ; L_{p}\left(\boldsymbol{R}_{+}^{n}\right)\right)_{\theta}$ est l'intersection des espaces.

$$
E=\left(\stackrel{\circ}{W}_{p}^{m}\left(\boldsymbol{R}_{+} ; L_{p}\left(\boldsymbol{R}^{n-1}\right)\right) ; L_{p}\left(\boldsymbol{R}_{+}^{n}\right)\right)_{\theta} \quad \text { et } \quad F=\left(L_{p}\left(\boldsymbol{R}_{+} ; W_{p}^{m}\left(\boldsymbol{R}^{n-1}\right)\right) ; L_{p}\left(\boldsymbol{R}_{+}^{n}\right)\right)_{\theta} .
$$

Le Théorème 2.10. appliqué avec $A=-\partial / \partial x_{n}$ implique que

$$
E=\left(\mathscr{W}_{p}^{1}\left(\boldsymbol{R}_{+} ; L_{p}\left(\boldsymbol{R}^{n-1}\right)\right) ; L_{p}\left(\boldsymbol{R}_{+}^{n}\right)\right)_{\sigma}
$$


tandis que si on l'applique avec

$$
A=\left[1-\sum_{j=1}^{n-1}\left(\frac{\partial}{\partial x_{j}}\right)^{2}\right]^{\frac{1}{2}}
$$

on obtient l'identité

$$
F=\left(L_{p}\left(\boldsymbol{R}_{+} ; W_{p}^{1}\left(\boldsymbol{R}^{n-1}\right)\right) ; L_{p}\left(\boldsymbol{R}_{+}^{n}\right)\right)_{\sigma} .
$$

On conclut cette première étape par une nouvelle application du Lemme 2.7. qui montre que

$$
E \cap F=\left(\stackrel{\circ}{W}_{p}^{1}\left(\boldsymbol{R}_{+}^{n}\right) ; I_{p}\left(\boldsymbol{R}_{+}^{n}\right)\right)_{\sigma}
$$

2ème étape. On explicite l'espace $\left(\stackrel{\circ}{W}_{p}^{1}\left(\boldsymbol{R}_{+}^{n}\right) ; L_{p}\left(\boldsymbol{R}_{+}^{n}\right)\right)_{\sigma}$ en appliquant les Théorèmes 2.8. et 2.6. avec

$$
\left\{\begin{array}{l}
A_{j}=+\partial / \partial x_{j}, \quad j=1, \ldots, n-1 \\
A_{n}=-\partial / \partial x_{n}
\end{array}\right.
$$

On voit ainsi que c'est le sous-espace de $L_{p}\left(\boldsymbol{R}_{+}^{n}\right)$ défini par les conditions

$$
\left\{\begin{array}{l}
\lim _{t \rightarrow 0} t^{-\sigma}\left(\int_{\boldsymbol{R}_{+}^{n}}\left|u\left(x+t e_{j}\right)-u(x)\right|^{p} d x\right)^{1 / p}=0, \quad j=1,2, \ldots, n-1 \\
\lim _{t \rightarrow 0} t^{-\sigma}\left(\int_{\boldsymbol{R}_{+}^{n}}\left|\tilde{u}\left(x-t e_{n}\right)-u(x)\right|^{p} d x\right)^{1 / p}=0
\end{array}\right.
$$

où $\tilde{u}$ est le prolongement de $u$ par zéro pour $x_{n}<0$.

Grâce à la Définition 6.1. on voit qu'il est équivalent d'exiger que $u \in h_{p}^{(\sigma)}\left(\boldsymbol{R}_{\leftarrow}^{u}\right)$ et que

$$
\lim _{t \rightarrow 0} t^{-\sigma}\left(\int_{0<\alpha_{n}<t}|u(x)|^{p} d x\right)=0
$$

On conclut pour $\sigma<1 / p$ à l'aide du

LEMME 6.4. - Pour $0<\sigma<1 / p$ les fonctions de $h_{p}^{\sigma}\left(\boldsymbol{R}_{+}^{n}\right)$ vérifient (6.1.).

Ce résultat n'est plus vrai pour $\sigma \geqslant 1 / p$ et au moins pour $\sigma>1 / p$ on peut vérifier que (6.1.) équivant ̀̀ $u\left(x_{1}, \ldots, x_{n-1}, 0\right)=0$.

Démonstratron. - On la fait dans le cas où $n=1$ pour simplifier l'écriture; le cas général se traite de manière analogue.

24 - Annali di Matematica 
On introduit

$$
\begin{aligned}
& F(t)=\left(\int_{0}^{t}|u(x)|^{p} d x\right)^{1 / p} \\
& G(t)=\left(\int_{0}^{t}|u(x+t)-u(x)|^{p} d x\right)^{1 / p} .
\end{aligned}
$$

Par hypothèse on sait que $t^{-\sigma} G(t) \rightarrow 0$ lorsque $t \rightarrow 0$ avee $0<\sigma p<1$ et il faut prouver que $t^{-\sigma} F(t) \rightarrow 0$ également lorsque $t \rightarrow 0$. Il est clair que

$$
\begin{aligned}
F(t) & =\left(\int_{0}^{t}|u(x+t)+u(x)-u(x+t)|^{p} d x\right)^{1 / p} \\
& \leqslant\left(\int_{0}^{t}|u(x+t)|^{p} d x\right)^{1 / p}+G(t) \\
& =\left(\int_{t}^{2 t}\left\{\left.u(x)\right|^{p} d x\right)^{1 / p}+G(t)\right.
\end{aligned}
$$

done que

$$
F(t) \leqslant\left(F(2 t)^{p}-F(t)^{p}\right)^{1 / p}+G(t)
$$

Soit $l$ la limite de $t^{-\sigma} F(t)$ lorsque $t \rightarrow 0$ en décroissant alors on a

$$
0 \leqslant l \leqslant\left(2^{\sigma D}-1\right)^{1 / p} l
$$

Ceci implique que $l=0$ dès que $1>2^{\sigma p}-1$ soit $2^{\sigma p}<2$ ce qui est le cas dès que $\sigma p<1$. C.Q.F.D.

A ce point le Théorème 6.3. est complètement démontré. On peut compléter cet énoncé en examinant le cas $\sigma=m(1-\theta)>1 / p$, ce qui nécessite un résultat préliminaire

Proposition 6.5. - Pour $\sigma>1 / p$ les fonetions de $h_{p}^{\sigma}\left(\boldsymbol{R}_{+}\right)$sont hoblderiennes d'expo$\operatorname{sant} \sigma-1 / p$.

Dhmongtration. - On utilise la formule de représentation de $u$ qu'on obtient ainsi:

$$
\begin{aligned}
& F(h)=\frac{1}{h} \int_{0}^{h} u(x+s) d s \quad(h>0) \\
& F(0)=F(h)-\int_{0}^{h} F^{\prime}(y) d y
\end{aligned}
$$


done

$$
\begin{aligned}
& u(x)=\frac{1}{h} \int_{0}^{h} u(x+s) d s-\int_{0}^{h} \frac{d y}{y^{2}} \int_{0}^{y}[u(x+y)-u(x+s)] d s \\
&=\frac{1}{h} \int_{0}^{h} u(x+s) d s-\int_{0}^{h} d s \int_{0}^{h}[u(x+y)-u(x+y-s)] \frac{d y}{y^{2}} .
\end{aligned}
$$

On en déduit que

$$
\begin{aligned}
u(x)-u(\xi) & =\frac{1}{h} \int_{0}^{h}[u(x+s)-u(\xi+s)] d s . \\
& -\int_{0}^{h} d s \int_{s}^{h}[u(x+u)-u(x+y-s)] \frac{d y}{y^{2}}+\int_{0}^{h} d s \int_{s}^{h}[u(\xi+y)-u(\xi+y-s)] \frac{d y}{y^{2}}
\end{aligned}
$$

d'où

$$
|u(x)-u(\xi)| \leqslant C\left\{h^{-1 / p}|x-\xi|^{\sigma}+2 h^{\sigma-1 / p} \int_{0}^{1} t^{\sigma}\left(1-t^{1-2 q}\right)^{1 / q} d t\right\}
$$

avec $1 / p+1 / q=1$ et en tenant compte de la définition de $h_{p}^{\sigma}\left(\boldsymbol{R}_{+}\right)$. On conclut en choisissant $h=|x-\xi|$ lorsque $\sigma-1 / p>0$.

CoRollaire 6.6. - Pour $1>\sigma>1 / p$ et $u \in h_{p}^{\sigma}\left(\boldsymbol{R}_{+}^{n}\right)$, on a (6.1.) si et seulement si $u\left(x_{1}, \ldots, x_{n_{-1}}, 0\right)=0$.

Démonstration. - On la fait dans le cas $n=1$ pour abréger. On suppose donc que $u \in h_{p}^{\sigma}\left(\boldsymbol{R}_{+}\right)$et $u(0)=0$.

Comme $W_{p}^{1}\left(\boldsymbol{R}_{+}\right)$est dense dans $h_{p}^{\sigma}\left(\boldsymbol{R}_{+}\right)$, on a

done

$$
|u(x)-u(\xi)|=\mathcal{O}\left(|x-\xi|^{\sigma-1 / p}\right)
$$

$$
u(x)=\mathcal{O}\left(x^{\sigma-1 / p}\right)
$$

On en déduit que

$$
t^{-\sigma}\left(\int_{0}^{t}|u(x)|^{p} d x\right)^{1 / p} \leqslant t^{-\sigma}\left(\int_{0}^{t}\left|\frac{u(x)}{x^{\sigma-1 / p}}\right|^{p} d x\right)^{1 / p} t^{\sigma-1 / p}=\left(\frac{1}{t} \int_{0}^{t}\left|\frac{u(x)}{x^{\sigma-1 / p}}\right|^{p} d x\right)^{1 / p} \rightarrow 0
$$

lorsque $t \rightarrow 0$, ce qui est la condition (6.1.).

Réciproquement si $u \in h_{p}^{\sigma}\left(\boldsymbol{R}_{+}\right)$on a

$$
t^{1 /) p-\sigma}\left(\int_{0}^{t}|u(x)|^{p} d x\right)^{1 / p} \sim|u(0)| t^{-\sigma}
$$

et par conséquent la condition (6.1.) implique $u(0)=0$. 
Le résultat de ce Lemme implique que pour $1>\sigma>1 / p$ l'espace $\left(\hat{W}_{p}^{1}\left(\boldsymbol{R}_{+}^{n}\right) ; L_{p}\left(\boldsymbol{R}_{+}^{n}\right)\right)_{\sigma}$ n'est autre que le sous-espace de $h_{p}\left(\boldsymbol{R}_{+}^{n}\right)$ défini par $\left.u\right|_{\alpha_{n}=0}$. En raisonnant comme dans le Théorème 6.3. on obtient ainsi le

THÉoRk̀ue 6.7. - Si $\Omega$ est borné et à frontière de classe $C^{m}$, V'espace $\left(\hat{W}_{p}^{m}(\Omega) ; L_{p}(\Omega)\right)_{\theta}$ coincide aveo le sous-espace de $h_{p}^{\circ}(\Omega)$ défini par les conditions $\partial^{i} u / \partial v^{j}=0$ sur $\Gamma$ pour $0 \leqslant j<\sigma-1 / p$ où $\sigma=m(1-\theta)$ est supposé tel que $\sigma-1 / p$ soit non entier.

Rrmarque 6.8. - Dans les énoncés précédents (Théorème 6.3, et 6.7.) 1'hypothèse que $\Omega$ est borné et de classe $C^{m}$ a permis de réduire la démonstration au cas particulier où $\Omega=\boldsymbol{R}_{+}^{n}$. Les mêmes techniques de démonstration permettent de résoudre le cas où $\Omega$ est un produit de droites et de demi-droites et done aussi le cas où $\Omega$ se déduit localement d'un tel modèle par un $C^{m}$ difféomorphisme. On obtient ainsi l'extension des Théorèmes 6.3. et 6.7 . au cas où $\Omega$ est un ouvert borné de $\boldsymbol{R}^{2}$ à frontière de classe $C^{m}$ par morceaux sans angle nul ou égal à $2 \pi$ (polygone curviligne) et au cas où $Q$ est un ouvert borné de $\boldsymbol{R}^{3}$ à frontière de classe $C^{m}$ par morceaux sans angle dièdre nul ou égal à $2 \pi$ et avec la condition qu'en chaque sommet seulement trois arêtes se rencontrent.

Exemple 6.9. - Les énoncés précédents ne permettent pas de déterminer $D_{\dot{A}}(\sigma / m)$ pour $\sigma>1 / p$ en toute généralité lorsque

$$
\stackrel{\circ}{W}_{p}^{m}(\Omega) \subseteq D_{A} \subseteq \stackrel{\circ}{W}_{p}^{m}(\Omega)
$$

(c.f. commentaire du Théorème 6.3.). Cependant voici un cas particulier Hilbertien où il est possible de conclure: on suppose que

$$
X=I_{2}(\Omega), \quad D_{A}=\stackrel{\circ}{W}_{2}^{2 m}(\Omega) \cap W_{2}^{m}(\Omega)
$$

et que

$$
A u=\left(-\Delta_{x}\right)^{m} u
$$

On sait alors que $D_{A^{\frac{1}{2}}}=\mathscr{W}_{2}^{m}(\Omega)$ et que $D_{A^{\frac{1}{2}}}$ est de classe $\frac{1}{2}$ entre $D_{A}$ et $X$. Par conséquent on a

$$
D_{A}(\sigma / 2 m)=D_{A^{\frac{1}{2}}}(\sigma / m)=\left(\check{\complement}_{2}^{m}(\Omega) ; L_{2}(\Omega)\right)_{\theta}
$$

avec $m(1-\theta)=\sigma$ e'est done le sous-espace de $h_{2}^{\sigma}(\Omega)$ défini par $\partial^{\jmath} u /\left.\partial v^{3}\right|_{T}=0$ pour $0 \leqslant j<\sigma-\frac{1}{2}$ pourvu que $\sigma-\frac{1}{2}$ ne soit pas entier.

De manière analogue si

$$
X=L_{2}(\Omega), \quad D_{A}=\left\{u_{i} \in W_{2}^{2 m}(\Omega) ;\left.\frac{\partial^{j} u}{\partial v^{j}}\right|_{\Gamma}=0, \quad m \leqslant j \leqslant 2 m-1\right\}
$$

et

$$
A u=\left(-\Delta_{x}\right)^{n} u .
$$

On a alors $D_{A^{\frac{1}{2}}}=W_{2}^{m}(\Omega)$ et partant $D_{A}(\sigma / 2 m)=h_{p}^{\sigma}(\Omega)$ si $0<\sigma<m$. 
Giuseppe Da Prato - PterRe Grisvard: Equations d'évolution abstraites, ete. 373

6.2. On expose pour terminer un procédé général pour déterminer $D_{A}(\sigma / m)$ qui

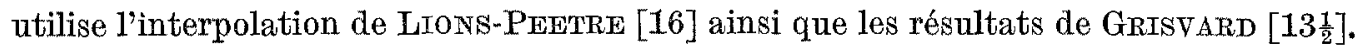

THÉORÈME 6.10. - Soit $X=L_{p}(\Omega)$ et

$$
D_{A}=\left\{u \in W_{p}^{m}(\Omega) ; B_{j} u=0 \text { sur } \Gamma, 1 \leqslant j \leqslant J\right\}
$$

où $\Omega$ est un ouvert borné de classe $O^{m}$ et $B_{j}, 1 \leqslant j \leqslant J$ un système normal d'opérateurs frontières sur $\Gamma$; soit $\beta_{j}$ l'ordre de $B_{j}$, alors

$$
D_{A}(\theta)=\left\{u \in h_{p}^{m \theta}(\Omega) ; B_{j} u=0 \text { sur } \Gamma \text { si } \beta_{j}<m \theta-\frac{1}{p}\right\}
$$

à condition qu'aueun opérateur $B_{j}$ ne soit d'ordre $m \theta-1 / p$ et que $m \theta$ soit non entier.

Le résultat analogue concernant les espaces d'interpolation habituels est le suivant: on note

$$
D_{A}(\theta ; p)=\left(D_{A} ; X\right)_{1-\theta ; p}
$$

l'espace d'interpolation défini de manière analogue aux Définitions 2.1. et 2.2. en remplaçant les fonctions continues dans $[0,1]$ par les fonctions mesurables et de puissance $p$ intégrable pour la mesure $d t / t$. Dans les hypothèses du Théorème 6.10. on a alors

$$
D_{A}(\theta ; p)=\left\{u \in W_{p}^{n \theta}(\Omega) ; \quad B_{j} u=0 \text { sur } \Gamma \text { si } \beta_{j}<m \theta-\frac{1}{p}\right\} .
$$

On va en déduire le Théorème 6.10. grâce à deux lemmess.

Lemae 6.11. $-(Y ; X)_{\theta / p}$ est de classe $\theta$ entre $Y$ et $X$.

DÉMonstration, - La condition (i) de la définition 2.13. est vérifiée dans LionsPeETRe [16]. En ce qui concerne la condition (ii), ces Auteurs établissent également que pour $\not{D} \in(Y ; X)_{\theta, p}$ il existe $u$ mesurable à valeurs dans $Y$ et continu à valeurs dans $X$ tel que $u(0)=x$ et

$$
\int_{0}^{+\infty} t^{\theta p}\|u(t)\|_{Y}^{p} \frac{d t}{t}<+\infty, \quad \int_{0}^{+\infty} t^{\theta p}\left\|u^{\prime}(t)\right\|_{Y}^{p} \frac{d t}{t}<+\infty .
$$
On a aussi $v(0)=x$ avec $v(t)=1 / t \int_{0}^{t} u(s) d s$ et il est facile de vérifier que $v \in O(\theta ; Y, X)$
done que $x \in(Y ; X)_{\theta}$.

LEMme 6.12. - Soit $\gamma$ une application linéaire continue de $X$ dans $Z$ où $Z$ est un espace de Banach et soient $Y_{\gamma}=\{y \in Y ; \gamma(y)=0\}, X_{\gamma}=\{x \in X ; \gamma(x)=0\}$. On suppose 
qu'it existe un projectewr $P$ linéaire continu de $Y$ sur $Y_{y}$ et de $X$ sur $X_{y}$, alors

$$
\left(Y_{\gamma} ; X_{\gamma}\right)_{\theta}=\left\{x \in(Y ; X)_{\theta} ; \gamma(x)=0\right\}
$$

Démonstramion. - L'inclusion de gauche à droite est évidente. Réciproquement si $x \in(Y ; X)_{\theta}$ et $\gamma(x)=0$, il existe $u \in C(\theta ; Y, X)$ avec $u(0)=x$; il est clair que puisque $\gamma(x)=0$, on a $P x=x=P u(0)$ et que $P u \in C\left(\theta ; Y_{\gamma}, X_{\gamma}\right)$; en conclusion $x \in\left(Y_{\gamma} ; X_{\gamma}\right)_{\theta}$.

Démonstratton dU ThÉorème 6.10. - On utilise le Lemme 6.12. et le Théorème 2.15. appliqué à $Z_{j}=\left(D_{A} ; X\right)_{1-\theta_{j} p}=D_{A}\left(\theta_{j}, p\right), j=0,1$ avec $\theta_{0}<\theta<\theta_{1}$; on a donc

$$
D_{A}(\theta)=\left(D_{A}\left(\theta_{1} ; p\right) ; D_{A}\left(\theta_{0} ; p\right)\right)_{\omega}
$$

pourvu que $\theta=(1-\omega) \theta_{0}+\omega \theta_{1}$.

Ensuite on utilise la caractérisation ci-dessus des espaces $D_{A}\left(\theta_{j} ; p\right)$ pour $j=0,1$, à savoir:

$$
D_{A}\left(\theta_{j}, p\right)=\left\{u \in W_{p}^{m \theta_{j}}(\Omega) ; B_{j} u=0 \text { sur } \Gamma \text { si } \beta_{j}<m \theta_{j}-\frac{1}{p}\right\}
$$

Comme on suppose que $m \theta-1 / p$ est non entier, on peut choisir $\theta_{0}$ et $\theta_{1}$ suffisamment proches de $\theta$ pour qu'il n'y ait aucun entier dans l'intervalle $\left[m \theta_{0}-1 / p\right.$; $\left.m \theta_{1}-1 / p\right]$. Dans ce cas $D_{A}\left(\theta_{j} ; p\right)$ est le noyau dans $W_{p}^{m \theta_{j}}(\Omega)$ de l'opérateur $\gamma$ défini par

$$
\gamma\left\{\begin{array}{l}
u \rightarrow\left\{\left.B_{j} u\right|_{X}\right\}_{\beta_{j}<m \theta_{0}-1 / p} \\
W_{p}^{m \theta_{j}}(\Omega) \rightarrow \prod_{\beta_{j}<m \theta_{0}-1 / p} W_{p}^{m \theta_{0}-\beta_{j}-1 / p}(\Gamma) .
\end{array}\right.
$$

On conclura en appliquant le Lemme 6.12. avec $Y=W_{p}^{m \theta_{1}}(\Omega)$ et $X=W_{g}^{m \theta_{0}}(\Omega)$ et $\gamma$ défini ci-dessus.

Sous réserve de vérifier la condition de projecteur on obtiendra done le résultat suivant: $D_{A}(\theta)$ est le noyau de $\gamma$ dans l'espace

$$
\left(W_{n}^{m \theta_{1}}(\Omega) ; W_{p}^{m \theta_{0}}(\Omega)\right)_{\omega}=E
$$

Comme $W_{p}^{m \theta_{j}}(\Omega)=\left(W_{p}^{m}(\Omega) ; L_{p}(\Omega)\right)_{1-\theta_{j} p}$, il est d'après le Lemme 6.11. de classe $1-\theta_{j}$ entre $W_{p}^{m}(\Omega)$ et $L_{p}(\Omega)$. Une nouvelle application du Théorème 2.15 . jointe à la Proposition 6.2., montre que $E=h_{y}^{m \theta}(\Omega)$ et par conséquent $D_{A}(\theta)$ est le noyau de $\gamma$ dans $h_{p}^{m \theta}(\Omega)$.

Ceci prouve le Théorème 6.10. sous réserve de vérifier la condition de projecteur.

Pour cela on utilise le résultat suivant qui est classique: pour tout $k$ il existe 
un opérateur $C_{b}$ linéaire continu de $W^{m \theta_{j}-1 / p-k}(\Gamma)$ dans $W_{p}^{m \theta_{j}}(\Omega)$ pour $j=0,1$ tel que

$$
\left\{\begin{array}{l}
\left.\frac{\partial^{k}}{\partial v^{k}} C_{k} f\right|_{\Gamma}=f \\
\left.\frac{\partial^{m}}{\partial v^{m}} C_{k} f\right|_{\Gamma}=0 \quad \text { si } m \neq k
\end{array}\right.
$$

avec $0 \leqslant m<m \theta_{0}-1 / p$. Pour la suite il est commode de supposer que les $B_{f}$ ont été numérotés de sorte que $j \rightarrow \beta_{j}$ soit croissant; par conséquent la condition $\beta_{j}<m \theta_{0}-1 / p$ équivaut à $j \leqslant l$ pour un certain $l$.

$$
\begin{cases}\left.B_{j} C_{k} f\right|_{T}=0 & \text { si } \beta_{j}<k \\ \left.B_{j} C_{\theta} f\right|_{T}=f & \text { si } \beta_{j}=k \\ \left.B_{j} C_{k} f\right|_{T}=R_{j}^{k} f & \text { si } \beta_{j}>k\end{cases}
$$

où $R_{i}^{k}$ est le coefficient de $(\partial / \partial v)^{k}$ dans l'opérateur $B_{j}$; c'est done un opérateur tangentiel à $\Gamma$ d'ordre $\leqslant \beta_{j}-\not$.

On définit alors une suite d'opérateurs en posant

$$
\left\{\begin{array}{l}
D_{1}\left\{f_{1}, \ldots, f\right\}=C_{\beta_{1}} f_{1} \\
\cdots \cdots \\
\left.D_{k}\left\{f_{1}, \ldots, f_{l}\right\}=D_{k-1}\left\{f_{1}, \ldots, f_{l}\right\}+C_{\beta_{k}}\left(f_{k}-B_{k} D_{k-1}\left\{f_{1}, \ldots, f_{l}\right\}\right)\right)_{\Gamma} \\
k=2, \ldots, l
\end{array}\right.
$$

C'est une suite d'opérateurs linéaires continus de

$$
\prod_{k=1}^{l} W_{p}^{n \theta_{j}-\beta_{k}-1 / p}(\Gamma) \quad \text { dans } \quad W_{p}^{m \theta_{j}}(\Omega)
$$

pour $j=0,1$, qui ont la propriété que

$$
\left\{\begin{array}{l}
\left.B_{j} D_{k}\left\{f_{1}, \ldots, f_{l}\right\}\right|_{\Gamma}=\left.B_{j} D_{k-1}\left\{f_{1}, \ldots, f_{l}\right\}\right|_{\Gamma} \quad \text { si } j<k \\
\left.B_{k} D_{k}\left\{f_{1}, \ldots, f_{l}\right\}\right|_{\Gamma}=f_{k}
\end{array}\right.
$$

done que

$$
\left.B_{j} D_{l}\left\{f_{1}, \ldots, f_{l}\right\}\right|_{\Gamma}=f_{j}, \quad j=1,2, \ldots, l .
$$

On obtient le projecteur désiré sur le noyau $\gamma$ en posant

$$
P u=u-D_{\imath}\left\{\left.B_{1} u\right|_{\Gamma}, \ldots,\left.B_{\imath} u\right|_{\Gamma}\right\} .
$$

C'est un opérateur linéaire continu dans $W_{p}^{m \theta_{j}}(\Omega)$ pour $j=0,1$ tel que $P u=u$ dès que $\gamma u=0$ c'est-à-dire dès que $B_{j} u=0$ sur $\Gamma$ pour $j=1,2, \ldots, l$. C.Q.F.D. 


\section{7. - Problèmes mixtes.}

7.1. On va d'abord résoudre des problèmes mixtes relatifs à des équations paraboliques non linéaires avec une seule variable d'espace. Ceci nécessite senlement la connaissance de la théorie spectrale des équations différentielles, théorie qui se développe sans difficulté dans l'espace des fonctions continues. Par conséquent ce point 7.1. n'utilisera pas les résultats du $\$ 6$.

Précisément on considèrera l'équation suivante

$$
\frac{\partial u}{\partial t}=a\left(t ; \frac{\partial^{2} u}{\partial x^{2}}, \frac{\partial u}{\partial x}, u\right)+f
$$

ò̀ $u$ (ineonnue) et $f$ (donnée) sont toutes deux fonctions de $t \in[0, T]$ et $x \in[0,1]$ et $a$ est une fonction numérique définie dans $[0, T] \times \boldsymbol{R}^{\mathbf{z}}$.

On va appliquer la théorie développée au $\mathrm{n}^{\circ} 4$ avec

et

$$
E=\{v \in C([0,1]) ; v(0)=v(1)=0\}, \quad \Lambda=\frac{d^{2}}{d x^{2}}
$$

$$
D=D_{\Lambda}=\left\{v \in O([0,1]) ; v(0)=v(1)=v^{\prime \prime}(0)=v^{\prime \prime}(1)=0\right\}
$$

Ce ehoix signifie qu'on envisage, dans un premier temps, la résolution d'un problème de Cauchy-Dirichlet par l'équation (7.1.).

Dans ces conditions $A$ est générateur d'un semi-groupe analytique fortement continu dans $E$. Il faut à présent déterminer l'espace $D_{A}(\theta)$. Pour cela il sera commode d'introduire de nouveaux espaces de Hölder qu'on notera $h_{\infty}^{\alpha}$ (par analogie avec les espaces de Nikolski du $\S 6$ ).

Définition 7.1. - Pour s réel $>0$ non entier $(s=k+\sigma$, $k$ entier, $0<\sigma<1)$ et $\Omega$ ouvert borné de $\boldsymbol{R}^{n}$, on désigne par $h_{\infty}^{(s)}(\bar{\Omega})$ le sous-espace de $C^{k}(\bar{\Omega})$ défini par les conditions

$$
t^{-\sigma} \operatorname{Max}_{\substack{x, y \in \bar{Q} \\|x-u| \leqslant t}}\left|D^{\alpha} u(x)-D^{\alpha} u(y)\right| \rightarrow 0
$$

lorsque $t \rightarrow 0$, pout tout multientier $\alpha$ de longueur $k$.

Cet espace est muni de la norme naturelle e'est-à-dire

$$
u \mapsto \sum_{|\alpha| \leqslant k} \operatorname{Max}_{x \in \bar{\Omega}}\left|D^{\alpha} u(x)\right|+\sum_{|\alpha|=k} \operatorname{Max}_{\substack{\alpha, y=\bar{\Omega}, l>0 \\|x-y| \leqslant t}} t^{-\sigma}\left|D^{\alpha} u(x)-D^{\alpha} u(y)\right| .
$$

Lemare 7.2. - Pour $\theta \neq 1 / 2$, on a $D_{A}(\theta)=E \cap h_{\infty}^{(2 \theta)}([0,1])$.

En d'autres termes c'est le sous-espace de $h_{\infty}^{(2 \theta)}([0,1])$ formé des fonctions $v$ telles que $v(0)=v(1)=0$. 
Démonstratron. - Par localisation on se ramène au cas où

$$
\begin{aligned}
& E=\{v \in C([0, \infty]) ; v(0)=v(\infty)=0\} \\
& D_{A}=\left\{v \in C^{2}([0, \infty]) ; v(0)=v^{\prime \prime}(0)=v(\infty)=v^{\prime}(\infty)=v^{\prime \prime}(\infty)=0\right\}
\end{aligned}
$$

Ensuite si on pose

$$
\begin{aligned}
& X=\{v \in O(\overline{\mathbf{R}}) ; v(-\infty)=v(\infty)=0\} \\
& Y=\left\{v \in C^{2}(\overline{\mathbf{R}}) ; v(-\infty)=v(+\infty)=v^{\prime}(-\infty)=v^{\prime}(+\infty)=v^{\prime \prime}(-\infty)=v^{\prime \prime}(+\infty)=0\right\}\left({ }^{14}\right)
\end{aligned}
$$

On voit en prolongeant les fonctions de $E$ et $D_{A}$ par imparité, que $E$ s'identifie au sous-espace des fonctions impaires dans $X$ et $D_{A}$ au sous-espace des fonctions impaires dans $Y$. En conséquence $D_{A}(\theta)$ est l'espace des restrictions à $[0, \infty]$ des fonctions impaires de $(Y ; X)_{1-\theta}$; (ceci est aussi une conséquence du Lemme 6.12.) il reste à identifier $(Y ; X)_{1-\theta}$; on remarque pour cela que $Y=D_{A^{2}}$ où $A=d / d x$ considéré dans l'espace $X$ avec domaine

$$
D_{A}=\left\{v \in O^{1}(\overline{\boldsymbol{R}}) ; v(-\infty)=v(+\infty)=v^{\prime}(-\infty)=v^{\prime}(+\infty)=0\right\}
$$

Comme $A$ est le générateur infinitésimal du groupe (fortement continu d'isométries) des translations dans $X$, le Théorème 2.6. et le Théorème 2.10. permettent alors d'identifier $\left(D_{A^{2}} ; X\right)_{1-\theta}$ comme étant

(i) $\left(D_{A} ; X\right)_{1-2 \theta} \quad$ si $0<\theta<\frac{1}{2}$;

(ii) $\left(D_{A^{2}} ; D_{A}\right)_{2-2 \theta} \quad$ si $\frac{1}{2}<\theta<1$;

c'est-à-dire

(i) le sous-espace de $X$ formé des $v$ tels que $t^{-2 \theta} \operatorname{Max}|v(x+t)-v(x)| \rightarrow 0$ lorsque $t \rightarrow 0$;

(ii) le sous-espace de $D_{A}$ formé des $v$ tels que

$$
t^{-(2 \theta-1)} \underset{\overline{\mathbf{R}}}{\operatorname{Max}}\left|v^{\prime}(x+t)-v^{\prime}(x)\right| \rightarrow 0 \quad \text { lorsque } t \rightarrow 0
$$

Dans les deux cas la condition d'imparité implique $v(0)=0$. C.Q.F.D.

Pour la suite on se limitera au cas $0<\theta<\frac{1}{2} ; D_{A}(\theta)$ est le sous-espace de $h_{\infty}^{(2 \theta)}([0,1])$ défini par les conditions $v(0)=v(1)=0$ et par conséquent $D_{A}(\theta+1)=$

(14) Ici $\bar{R}=[-\infty,+\infty]$. 
$=\left\{v \in D_{A} ; A v \in D_{A}(\theta)\right\}$ est le sous-espace de $h_{\infty}^{(2 \theta+2)}([0,1])$ défini par les conditions $v(0)=v^{\prime \prime}(0)=v(1)=v^{\prime \prime}(1)=0$.

Ceci étant on pose

$$
\varphi(t, v)(x)=a\left(t ; v^{\prime \prime}(x), v^{\prime}(x), v(x)\right)+f(t ; x) .
$$

On suppose que $f$ est continue dans $[0, T]$ à valeurs dans $D_{A}(\theta)$ et on supposera que la fonction

$$
t, s_{1}, s_{2}, s_{3} \mapsto a\left(t ; s_{1}, s_{2}, s_{3}\right)
$$

admet des dérivées jusqu'à l'ordre deux par rapport à $s=\left(s_{1}, s_{2}, s_{3}\right)$, continues et $t$ et $s$ pour $t \in[0, T]$ et $s \in \boldsymbol{R}^{3}$. Enfin on suppose que $a\left(t ; 0, s_{2}, 0\right)=0$ pour tout $t \in[0, T]$ et $s_{2} \in \boldsymbol{R}$. Il est alors clair que

$$
\varphi \in C\left([0, T] \times D_{A}(\theta+1) ; D_{A}(\theta)\right)
$$

C'est l'hypothèse (4.1.) point (i). Ensuite on a

$$
\frac{\partial \varphi}{\partial v}(t ; v)=\sum_{j=1}^{3} \frac{\partial a}{\partial s_{j}}\left(t ; v^{\prime \prime}(x), v^{\prime}(x), v(x)\right)\left(\frac{d}{d x}\right)^{3-j}
$$

il est clair que

$$
\frac{\partial \varphi}{\partial v} \in C\left([0, T] \times D_{\Lambda}(\theta+1) ; L\left(D_{\Lambda}(\theta+1) ; D_{A}(\theta)\right)\right)
$$

e'est l'hypothèse (4.1.) point (ii)). Si on suppose de plus que $\left(\partial a / \partial s_{1}\right)(t ; s)>0$ pour tout $t \in[0, T]$ et $s \in \boldsymbol{R}^{3}$, alors $(\partial \varphi / \partial v)(t ; v)$ est générateur infinitésimal d'un semigroupe fortement continu analytique dans $E$, de domaine $D_{A}$ grâce au

LEMME 7.3. - Soient $b_{j} \in C([0,1]), j=1,2,3$ avee $b_{1}(x) \geqslant \alpha>0$ pour tout $x \in[0,1]$ et $b_{2}(0)=b_{2}(1)=0$, alors l'opérateur $B=b_{1}\left(d^{2} / d x^{2}\right)+b_{2}(d / d x)+b_{3}$ de domaine $D_{A}$ dans $E$, est générateur infinitésimal d'un semi-groupe analytique fortement continu.

Démonstration. - $B$ est évidemment fermé et à domaine dense; il faut seulement localiser l'ensemble résolvant de $B$ et préciser la croissance de sa résolvante. L'équation $B v-\lambda v=g$ avee $v \in D_{A}$, équivaut à

$$
b_{1} v^{\prime \prime}+b_{2} v^{\prime}+b_{3} v-\lambda v=g
$$

dans $[0,1]$ avec les conditions $v(0)=v(1)=0, g$ étant donné par hypothèse avec $g(0)=g(1)=0 \quad$ (condition qui impliquera automatiquement $v^{\prime \prime}(0)=v^{\prime \prime}(\mathbf{1})=0$ puisque $b_{1}$ ne s'annulle pas et $\left.b_{2}(0)=b_{2}(1)=0\right)$. 
On peut réécrire cette équation sous la forme

$$
v^{\prime \prime}-\frac{\lambda}{b_{1}(0)} v=\frac{b_{1}(0)-b_{1}}{b_{1}(0)} v^{\prime \prime}-\frac{b_{2}}{b_{1}(0)} v^{\prime}-\frac{b_{3}}{b_{1}(0)} v+\frac{g}{b_{1}(0)}
$$

d'où, en posant $v^{\prime \prime}-\left(\lambda / b_{1}(0)\right) v=h$, on déduit

$$
h=\left\{\frac{b_{1}(0)-b_{1}}{b_{1}(0)} A-\frac{b_{2}}{b_{1}(0)} \frac{d}{d x}-\frac{b_{3}}{b_{1}(0)}\right\}\left(\Lambda-\frac{\lambda}{b_{1}(0)}\right)^{-1} h+\frac{1}{b_{1}(0)} g
$$

à condition que $\lambda / b_{1}(0)$ soit dans l'ensemble résolvant de $A$ c'est-à-dire en particulier si $\lambda \in[-\infty, 0]$. On a alors l'existence et l'unicité de $h$ (done de $v$ ) lorsque

$$
\left\|\left\{\frac{b_{1}(0)-b_{1}}{b_{1}(0)} A-\frac{b_{2}}{b_{1}(0)} \frac{d}{d x}-\frac{b_{3}}{b_{1}(0)}\right\}\left(A-\frac{\lambda}{b_{1}(0)}\right)^{-1}\right\|_{E \rightarrow E}<1 .
$$

Pou vérifier cette condition on utilise la majoration

$$
\left\|(\Lambda-\mu)^{-1}\right\|_{E \rightarrow E} \leqslant \frac{1}{-\mu \mid \cos \theta / 2}
$$

pour $\arg \mu=\theta \in]-\pi,+\pi[$, d'où

$$
\left\|\Lambda(\Lambda-\mu)^{-1}\right\|_{E \rightarrow E} \leqslant \frac{1}{\cos \theta / 2}
$$

et (en majorant grossièrement)

$$
\left\|\frac{d}{d x}(\Lambda-\mu)^{-1}\right\|_{E \rightarrow-E} \leqslant \frac{K}{|\mu|^{\frac{x}{3}} \cos 0 / 2}
$$

où la valeur exacte de $K$ (indépendante de $\mu$ ) importe peu dans ce qui suit. On en déduit la majoration

$$
\begin{aligned}
\|\left\{\frac{b_{1}(0)-b_{1}}{b_{1}(0)} A-\frac{b_{2}}{b_{1}(0)}\right. & \left.\frac{d}{d x}-\frac{b_{3}}{b_{1}(0)}\right\}\left(A-\frac{\lambda}{b_{1}(0)}\right)^{-1} \|_{E \rightarrow E} \\
& \leqslant \frac{1}{\cos \theta / 2}\left\{\operatorname{Max}_{[0,1]} \frac{\left|b_{1}(0)-b_{1}\right|}{\left|b_{1}(0)\right|}+\underset{[0,1]}{\operatorname{Max}} \frac{\left|b_{2}\right|}{\left|b_{1}(0)\right|^{\frac{2}{2}}} \frac{K}{|\lambda|^{\frac{1}{2}}}+\operatorname{Max}_{[0,1]}\left|b_{3}\right| \frac{1}{|\lambda|}\right\} \leqslant \frac{1}{2}
\end{aligned}
$$

à condition que $\arg \lambda=\theta \in\left[-\theta_{0},+\theta_{0}\right]$ où $\theta_{0}<\pi$ et

$$
\left\{\begin{array}{l}
\operatorname{Max}_{x \in[0,1]} 1-\frac{b_{1}(x)}{b_{1}(0)} \leqslant \frac{\cos \theta_{0} / 2}{4} \\
|\lambda|^{\frac{1}{2}} \geqslant \frac{8 \pi}{b_{1}(0)^{\frac{1}{2}}} \frac{1}{\cos \theta_{0} / 2} \operatorname{Max}_{x \in[0,1]}\left|b_{2}(x)\right| \\
|\lambda| \geqslant \frac{8}{\cos \theta_{0} / 2} \operatorname{Max}_{x \in[0,1]}\left|b_{3}(x)\right|
\end{array}\right.
$$


Pour ces mêmes $\lambda$ on en déduit l'inégalité

d'où

$$
\|h\|_{E} \leqslant \frac{2}{\left|b_{1}(0)\right|}\|g\|_{E}
$$

$$
\|v\|_{E} \leqslant \frac{2}{|\lambda| \cos \theta / 2}\|g\|_{E}
$$

on a ainsi prouvé que

$$
\left\|(B-\lambda)^{-1}\right\|_{E \rightarrow E} \leqslant \frac{2}{|\lambda| \cos \theta / 2}
$$

pour $|\lambda|$ assez grand avec $\arg \lambda=\theta \in\left[-\theta_{0}+\theta_{0}\right]$ pourvu que l'oscillation de $b_{1}$ dans $[0,1]$ soit assez petite e'est-à-dire

$$
\operatorname{Max}_{x \in[0,1]}\left|1-\frac{b_{1}(x)}{b_{1}(0)}\right| \leqslant \frac{\cos \theta_{0} / 2}{4}
$$

en conséquence dans ce cas, $B$ est le générateur infinitésimal d'un semi-groupe analytique fortement continu dans $E$.

On ramène le cas général au cas particulier ci-dessus par l'artifice de Korn, c'està-dire par partition de l'unité. C.Q.F.D.

A ce point on sait déjà que les hypothèses (4.1.) (i), (ii) et (iii) sont vérifiées. Il reste à déterminer $D_{B}(\theta+1)$ lorsque $B=(\hat{\partial} \varphi / \partial v)(t, v)$ c'est-à-dire

$$
b_{j}(x)=\frac{\partial a}{\partial s_{j}}\left(t ; v^{\prime \prime}(x), v^{\prime}(x), v(x)\right), \quad j=1,2,3
$$

Par définition on a

$$
D_{B}(\theta+1)=\left\{w \in D_{B} ; B w \in D_{B}(\theta)\right\} .
$$

Ici on a $D_{B}=D_{A}$ avec équivalence des normes, donc $D_{B}(\theta)=D_{A}(\theta)$ par interpolation. La condition $B w \in D_{B}(\theta)$ équivant done puisque $0<\theta<\frac{1}{2} \grave{a} b_{1} w^{\prime \prime} \in D_{A}(\theta)$ et pax conséquent à $A w=w^{\prime \prime} \in D_{A}(\theta)$ si l'espace $D_{A}(\theta)$ est stable par multiplication par $1 / b_{1}$; c'est le cas puisque $b_{1}$ est holdérienne d'exposant $2 \theta$ au sens strict (c'est-à-dire au sens de $\left.h_{\infty}^{(2 \theta)}([0,1])\right)$.

En récapitulant, l'application du Théorème 4.1. donne le

ThÉoRk̀Mre 7.3. - On suppose que $t, s \mapsto a\left(t ; s_{1}, s_{2}, s_{3}\right)$ admet des dérivées jusqu'à l'ordre deux continues en $t \in[0, T]$ et $s \in \boldsymbol{R}^{3}$, que a $\left(t ; 0, s_{2}, 0\right)=0$ pour tout $t \in[0, T]$ et tout $s_{2} \in \boldsymbol{R}$ et que $\left(\partial a / \partial s_{1}\right)(t ; s)>0$ pour tout $t \in[0, T]$ et tout $s \in \boldsymbol{R}^{3}$. Soit également $\varrho \in] 0,1\left[\right.$. Alors pour tout $u_{0} \in h_{\infty}^{(\varrho+2)}([0,1])$ avec $u_{0}(0)=u_{0}(1)=u_{0}^{\prime \prime}(0)=u_{0}^{\prime \prime}(1)=0$ et 
tout $f \in C\left([0, T] ; h_{\infty}^{(o)}([0,1])\right)$ vérifiant $f(t, 0)=f(t, 1)=0$, il existe $\tau>0$ et une unique $u \in C\left([0, \tau] ; h_{\infty}^{(\varrho+2)}([0,1])\right) \cap C^{1}\left([0, \tau] ; h_{\infty}^{(\varrho)}([0,1])\right)$ solution de l'équation (7.1.) vérifiant les conditions

$$
\begin{cases}u(0, x)=u_{0}(x), & x \in[0,1] \\ u(t, 0)=u(t, 1)=0, & t \in[0, T]\end{cases}
$$

Le résultat précédent est relatif au problème de Cauchy-Dirichlet pour l'équation (7.1.). Pour résoudre un problème de Cauchy-Neuman il suffit de poser

$$
E=C([0,1]), \quad \Lambda=\frac{d^{2}}{d x^{2}}
$$

$D_{A}=\left\{v \in C^{2}([0,1]) ;-v^{\prime}(1)=\alpha v(1), v^{\prime}(0)=\beta v(0)\right\}$ où $\alpha$ et $\beta$ sont deux réels non négatifs fixés. Des raisonnements analogues à ceux qui précèdent, montrent que

$$
D_{A}(\theta)=h_{\infty}^{2 \theta}([0,1]) \quad \text { si } 2 \theta<1 \text {. }
$$

L'application du Théorème 4.1. conduit au

THÉozìme 7.4. - On suppose que $t, s \mapsto a\left(t, s_{1}, s_{2}, s_{3}\right)$ admet des dérivées jusqu'à l'ordre deux continues en $t \in[0, T]$ et $s \in \boldsymbol{R}^{3}$ et que $\left(\partial a / \partial s_{1}\right)(t, s)>0$ partout.

Soit $\varrho \in] 0,1\left[\right.$, alors pour tout $u_{0} \in h_{\infty}^{\alpha+2}([0,1])$ avec $-u_{0}^{\prime}(1)=\alpha u_{0}(1), u_{0}^{\prime}(0)=\beta u_{0}(0)$ et tout $f \in C\left([0, T] ; h_{\infty}^{e}([0,1])\right)$, il existe $\tau>0$ et une unique $u \in C\left([0, \tau] ; h_{\infty}^{0+2}([0,1])\right) \cap$ $\cap C^{1}\left([0, T] ; h_{\infty}^{e}([0,1])\right)$ solution de $(7,1)$ vérifiant les conditions

$$
\begin{cases}u(0, x)=u_{0}(x), & x \in[0,1] \\ -\frac{\partial u}{\partial x}(t, 1)=\alpha u(t, 1), & t \in[0, \tau] \\ \frac{\partial u}{\partial x}(t, 0)=\beta u(t, 0), & t \in[0, \tau] .\end{cases}
$$

7.2. On va maintenant étudier des problèmes mixtes relatifs à des équations paraboliques non linéaires avec plusieurs variables d'espace. Ceci nécessitera la connaissance de la théorie spectrale des problèmes aux limites elliptiques, qui, suivant Agmon [1] se développe bien dans l'espace des fonctions de puissance $p$ intégrable.

C'est pourquoi on ne pourra pas répéter exactement ce qu'on a fait en 7.1. pour les problèmes à une variable d'espace et on utilisera ici les résultats de $\$ 6$.

La méthode utilisée est applicable à des équations de la forme

$$
\frac{\partial u}{\partial t}=a\left(t, u, \nabla u, \nabla^{2} u\right)+f
$$


382 Giuseppe Da Prato - Pterre Grisvard: Equations d'évolution abstraites, ete.

où $\nabla$ désigne le gradient. Cependant pour éviter des complications dans les notations, on se contentera des équations de la forme

$$
\frac{\partial u}{\partial t}=a(t, u, \nabla u, \Delta u)+f
$$

où

$$
t, u, p, q \mapsto a(t, u, p, q)
$$

est une fonction de $[0, T] \times \boldsymbol{R} \times \boldsymbol{R}^{n} \times \boldsymbol{R}$.

On appliquera dans un premier temps la théorie du $\S 4$ avec $E=L_{p}(\Omega)$ où $\Omega$ est un ouvert borné de $\boldsymbol{R}^{n}$ à frontière $\Gamma$ régulière, $A<p<\infty$ et $A=\Delta$ considéré avec le domaine

$$
D_{A}=\left\{u \in W_{p}^{2}(\Omega) ; u=0 \text { sur } \Gamma\right\}
$$

Ce choix signifie qu'on envisage d'abord la résolution du problème de OauchyDirichlet relatif à l'équation (7.2.).

D'après AGron [1], $A$ est le générateur infinitésimal d'un semi-groupe analytique. Les espaces $D_{A}(\theta)$ ont été déterminés au $n^{\circ} 6$. D'après le Théorème 6.3. on a

$$
D_{A}(\theta)=h_{p}^{2 \theta}(\Omega) \quad \text { si } \theta<1 / 2 p
$$

tandis que d'après le Théorème 6.10. on a

$$
D_{A}(\theta)=\left\{u \in h_{p}^{2 \theta}(\Omega) ; u=0 \text { sur } \Gamma\right\} \quad \text { si } \frac{1}{2 p}<\theta<1 .
$$

On en déduit que

$$
\begin{array}{ll}
D_{A}(\theta+1)=\left\{u \in h_{p}^{2 \theta+2}(\Omega) ; u=0 \text { sur } \Gamma\right\} & \text { si } 0<\theta<\frac{1}{2 p} \\
D_{\Lambda}(\theta+1)=\left\{u \in h_{p}^{2 \theta+2}(\Omega) ; u=\Delta u=0 \text { sur } \Gamma\right\} & \text { si } \frac{1}{2 p}<\theta<1 .
\end{array}
$$

Cela étant on posera

$$
\varphi(t, u)=a(t, u, \nabla u, \Delta u),
$$

Pour vérifier les hypothèses du Théorème 4.1. il faut auparavant déterminer des conditions suffisantes pour q'une fonction opère dans un espace de Sobolev ou un espace de Nikolski. On utilisera pour cela quelques lemmes:

Lemue 7.4. - Soit $f$ une fonetion numérique continue définie dans $\boldsymbol{R}, m$ fois continument dérivable, alors $u \mapsto f \circ u$ est continu dans $W_{p}^{m}(\Omega)$ si $m>n / p$. 
Giuseppe Da Prato - Pierre Grisvard: Equations d'évolution abstraites, ete. 383

Ce résultat se vérifie par un calcul direct grâce aux théorèmes d'immersion de Sobolev.

LEMnE 7.5. - Soit $f$ une application localement Lipschitzienne de $\boldsymbol{R}$ dans $\boldsymbol{R}$, alors $u \mapsto f \circ u$ est continu dans $h_{p}^{s}(\Omega)$ si $1>s>n / p$.

Démonstration. - Grâce à l'hypothèse $s>n / p, h_{p}^{s}(\Omega)$ s'injecte continûment dans l'espace $C(\bar{\Omega})$ des fonctions continues sur $\bar{\Omega}$.

En effet par définition $u$ est la restriction à $\Omega$ d'une fonction $U \in h_{p}^{s}\left(\boldsymbol{R}^{n}\right)$. On voit aisément sur la Définition 6.1. que $U \in W_{p}^{s^{\prime}}\left(\boldsymbol{R}^{n}\right)$, pour $s^{\prime}<s$, par conséquent grâce au Théorème de Sobolev, $U$ est continu puisque $s>n / p$.

Il en résulte que $f \circ u$ est continue dans $\bar{Q}$ donc aussi élément de $L_{p}(\Omega)$ puisque $\Omega$ est borné.

Ensuite on a

$$
t^{-s p} \int_{\mathbf{R}^{n}}\left|f\left(u\left(x+t e_{j}\right)\right)-f(u(x))\right|^{p} d x \leqslant K t^{-s p} \int_{\boldsymbol{R}^{n}}\left|u\left(x+t e_{j}\right)-u(x)\right|^{p} d x \rightarrow 0
$$

lorsque $t \rightarrow 0$, où $K$ est la constante de Lipschitz de $f$ restreinte à l'image (bornée) de $u$. Ceci prouve que fo $u \in h_{p}^{s}(\Omega)$. La démonstration de la continuité de $u \rightarrow f \circ u$ suit les mêmes idées.

Les considérations ci-dessus s'étendent à des fonctions $f$ de plusieurs arguments; on utilisera le

LeMme 7.6. - Soit $f$ une application localement lipschitzienne de $\boldsymbol{R}^{v}$ dans $\boldsymbol{R}$, alors $\left\{u_{1}, \ldots, u_{\nu}\right\} \rightarrow f\left(u_{1}, \ldots, u_{\nu}\right)$ est continu de $h_{p}^{s}(\Omega)^{\nu}$ dans $h_{p}^{s}(\Omega)$ pour $1>s>n / p$.

De ce Lemme on déduit immédiatement que si a est localement lipschitzienne par rapport à $(u, p, q) \in \boldsymbol{R} \dot{\times} \boldsymbol{R}^{n} \times \boldsymbol{R}$, alors

$$
u \mapsto a(t, u, \nabla u, \Delta u)
$$

est pour chaque $t$ une application continue de $h_{p}^{s+2}(\Omega)$ dans $h_{p}^{s}(\Omega)$ pour $1>s>n / p$.

Ceci nous conduit pour appliquer la théorie du $\S 4$, à choisir $\theta$ tel que $1>2 \theta>n / p$; ceci suppose done $n>p$ et implique $1 / 2 p<\theta$ et partant, la condition de Dirichlet intervient dans la description de $D_{A}(\theta)$.

Cependant pour que l'ensemble des hypothèses (4.1.) soit satisfait on supposera ce qui suit sur a:

(i) a est continue, admet des dérivées premières en $u, p, q$ qui sont continues en $t$ et localement lipschitziennes en $u, p, q$.

(ii) $a(t, 0, p, 0)=0$ pour tout $t$ et tout $p$.

(iii) $(\partial a / \partial q)(t, u, p, q)>0$ partout. 
Giuseppe Da Prato - Pierre Grtsvard: Equations d'évolution abstraites, ete.

La vérification de (4.1.) utilisera le

LEMME 7.7. $-h_{p}^{s}(\Omega)$ est une algèbre pour $1>s>n / p$.

Démonstratron. - Il s'agit d'une simple vérification: soient $u$ et $v$ dans $h_{p}^{3}(\Omega)$ done respectivement restrietions de $U$ et $V$ éléments de $h_{p}^{s}\left(\boldsymbol{R}^{n}\right)$. Comme $\Omega$ est borné on peut supposer que $U$ et $V$ sont à supports compacts.

On a déjà remarqué que grâce à l'hypothèse $s>n / p, U$ et $V$ sont continues. Il en résulte immédiatement que $U V \in L_{p}\left(\boldsymbol{R}^{n}\right)$. D'autre part

$$
\begin{aligned}
\left(t^{-s p} \int_{\boldsymbol{R}^{n}}\left|U V\left(x+t e_{j}\right)-U V(x)\right|^{p} d x\right)^{1 / p} & \leqslant\left(t^{-s p} \int_{\boldsymbol{R}^{n}}\left|V\left(x+t e_{j}\right)-V(x)\right|^{p} d x\right)^{1 / p} \max _{x \in \mathbf{R}^{n}}|U(x)| \\
& +\left(t^{-s p} \int_{\boldsymbol{R}^{n}}\left|U\left(x+t e_{j}\right)-U(x)\right|^{p} d x\right)^{1 / p} \max _{x \in \mathbf{R}^{n}}|V(x)| \rightarrow 0
\end{aligned}
$$

lorsque $t \rightarrow 0$. Ceci prouve que $U V \in h_{p}^{s}\left(\boldsymbol{R}^{n}\right)$ et par restriction $u v \in h_{p}^{s}(\Omega)$ et de plus $u, v \mapsto u v$ est évidemment continu. O.Q.F.D.

A ce point de (i) il découle grâce aux Lemmes 7.6. et 7.7. que $\varphi$ définie en (7.3.) vérifie les conditions

$$
\varphi \in C\left([0, T] \times h_{p}^{2 \theta+2}(\Omega) ; h_{p}^{2 \theta}(\Omega)\right)
$$

et

$$
\frac{\partial \varphi}{\partial x} \in C\left([0, T] h_{p}^{2 \theta+2}(\Omega) ; L\left(h_{p}^{2 \theta+2}(\Omega) ; h_{p}^{2 \theta}(\Omega)\right)\right)
$$

avec

$$
\frac{\partial \varphi}{\partial x}(t, u) \cdot v=\frac{\partial a}{\partial u}(t, u, \nabla u, \Delta u) v+\frac{\partial a}{\partial p}(t, u, \nabla u, \Delta u) \cdot \nabla v+\frac{\partial a}{\partial q}(t, u, \nabla u, \Delta u) \Delta v .
$$

On en déduit (4.1.) (i) et (ii) grâce à (7.4.) (ii) car alors si $u=\Delta u=0$ sur $\Gamma=\partial \Omega$ on a

$$
a(t, u, \nabla u, \Delta u)=0 \quad \text { sur } \Gamma
$$

et si de plus $v=\Delta v=0$ sur $\Gamma=\partial \Omega$ on a aussi $(\partial \varphi / \partial x)(t, u) \cdot v=0$ sur $\Gamma$.

L'hypothèse $(4,1)$ (iii) résulte de (7.4.) (iii) car pour $u \in h_{y}^{2 \theta+2}(\Omega)$ on $a u, \nabla u$, $\Delta u \in C(\bar{\Omega})$ done le coefficient $(\partial a / \partial q)(t, u, \nabla u, \Delta u)$ est borné inférieurement par une quantité strictement positive dans $\bar{\Omega}$ et les résultats d'AGMon [1] sont utilisables.

Enfine pour vérifier (4.1.) (iv) on remarque que par définition

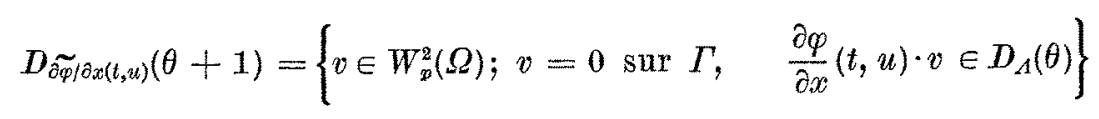


Giuseppe Da Prato - Pierre Grisvard: Equations d'évolution abstraites, etc. 385

c'est à dire que entre autres

$$
v \in W_{p}^{2}(\Omega), \quad \frac{\partial a}{\partial q}(t, u, \nabla u, \Delta u) \Delta v \in h_{p}^{2 \theta}(\Omega) ;
$$

comme $(\partial a / \partial q)(t, u, \nabla u, \Delta u) \in h_{p}^{2 \theta}(\Omega)$ et ne s'annule pas, il résulte du Lemme 7.7. que

$$
\Delta \theta \in h_{p}^{2}(\Omega)
$$

done

$$
\Delta v \in h_{p}^{2 \theta+2}(\Omega)
$$

grâce aux résultats de régularité de AgMron-NovaLIS-NIRENBERg [2].

En ce qui concerne la condition aux limites contenue dans la définition de $D_{A}(\theta)$ elle équivaut à

$$
\frac{\partial \varphi}{\partial x}(t, u) v=0 \quad \text { sur } \Gamma
$$

cependant grâce à (ii) on a

$$
\frac{\partial \varphi}{\partial x}(t, u) v=\frac{\partial a}{\partial q}(t, 0, \nabla u, 0) \Delta v=0 \quad \text { sur } \Gamma
$$

ce qui revient au même que $\Delta v=0$ sur $T$. En conclusion $v \in D_{(\tilde{\partial \varphi} / \partial x)(t, u)}(\theta+1)$ si et seulement si $v \in h_{p}^{2 \theta+2}(\Omega)$ et $v=\Delta v=0$ sur $T$ c'est-à-dire $v \in D_{A}(\theta+1)$.

A ce point l'application du Théorème 4.1. fournit le

THর́onł̀me 7.8. - On suppose que la fonotion a vérifie les conditions de l'Hypothèse (7.4.) (i) (ii) (iii). Soient

$p \in] n, \infty[$ et $s \in] n / p, \mathbb{1}\left[\right.$. Alors pour tout $u_{0} \in h_{p}^{s+2}(\Omega)$ avec $u_{0}=\Delta u_{0}=0$ sur $\Gamma$ et pour tout $f \in C\left([0, T] ; h_{p}^{s}(\Omega)\right)$ vérifiant $f(t, x)=0$ pour $t \in[0, T]$ et $x \in \Gamma$, rl existe $\tau>0$ et une unique $u \in C\left([0, \tau] ; h_{p}^{s+2}(\Omega)\right) \cap C^{1}\left([0, \tau] ; h_{p}^{s}(\Omega)\right)$ solution de l'équation $(7,2)$ vérifiant les conditions

$$
\left\{\begin{array}{l}
u(0, x)=u_{0}(x), \quad x \in \Omega \\
u(t, x)=0, \quad t \in[0, \tau], \quad x \in \Gamma .
\end{array}\right.
$$

On a ainsi résolu localement en temps le problème mixte de type Cauchy-Dirichlet relatif à l'équation (7.2.). La résolution d'un problème de type Cauchy-Neuman est partiellement plus simple. En effet soit $y$ la normale sortante à $\Gamma$ et $q$ une fonction régulière non négative sur $\Gamma$, on pose alors

$$
\Lambda=\Delta \quad D_{A}=\left\{u \in W_{p}^{2}(\Omega) ;-\frac{\partial u}{\partial \nu}=q u \text { sur } \Gamma\right\} .
$$

25 - Annali di Matematica 
Toujours d'après AGMoN [1], $A$ est générateur infinitésimal d'un semi-groupe analytique dans $B=L_{p}(\Omega)$ et on a

$$
\begin{gathered}
D_{\Lambda}(\theta)=h_{\nu}^{2 \theta}(\Omega) \quad \text { pour } 0<2 \theta<1+\frac{1}{p}, \\
D_{\Lambda}(\theta+1)=\left\{u \in h_{p}^{2 \theta+2}(\Omega) ;-\frac{\partial u}{\partial \nu}=q u \text { sur } \Gamma\right\} \quad \text { pour } 0<2 \theta<1+\frac{1}{p} .
\end{gathered}
$$

Ensuite définissant $\varphi$ toujours par (7.4.), on voit en appliquant les Lemmes 7.5. et 7.6. que $\varphi$ opère de $h_{n}^{s+2}(\Omega)$ dans $h_{p}^{s}(\Omega)$ toujours pour $1>s>n / p$.

On pourra donc travailler dans $D_{A}(\theta)$ avec $n / p<2 \theta<1(p>n)$, qui est un espace de Nikolski sans conditions aux limites, situation plus simple que dans le cas des conditions de Dirichlet.

L'application du Théorème 4.1. fournit le

THÉORÈMe 7.9. - On suppose que a vérifie (7.4.) (i) (iii). Soient $p \in] n, \infty[$ et $s \in] n / p, \mathbb{I}\left[\right.$. Alors pour tout $u_{0} \in h_{y}^{s+2}(\Omega)$ avec - $-\partial u_{0} / \partial v=q u_{0}$ sur $\Gamma$ et pour tout $f \in$ $\in C\left([0, T], h_{p}^{s}(\Omega)\right)$, il existe $\tau>0$ et une unique $u \in C\left([0, \tau] ; h_{p}^{s+2}(\Omega)\right) \cap O^{1}\left([0, \tau] ; h_{p}^{s}(\Omega)\right)$ solution de l'équation (7.2.) vérifiant les conditions

$$
\left\{\begin{array}{l}
u(0, x)=u_{0}(x), \quad x \in \Omega \\
-\frac{\partial u}{\partial v}(t, x)=q(x) u(t, x), \quad t \in[0, \tau], \quad x \in \Gamma .
\end{array}\right.
$$

7.3. Les conditions sur $a$ qui sont en hypothèse dans le Théorème 7.11. sont naturelles puisqu'il s'agit d'exiger que $a$ soit assez régulière et que $\partial a / \partial q$ soit strictement positive c'est-à-dire que l'opérateur considéré soit parabolique. Par contre dans le Théorème 7.10. qui est relatif aux conditions de Dirichlet, il intervient la condition (7.4.) (ii) qui est peut naturelle; elle est nécessaire car l'espace $D_{A}(\theta)$ fait intervenir la condition de Dirichlet. On peut cependant pour certains opérateurs de forme particulière travailler avee $\theta$ assez petit pour que la condition de Dirichlet disparaisse de la définition de $D_{A}(\theta)$ et alors l'hypothèse (7.4.) (ii) disparaitra. C'est ce qu'on va développer ici. On s'intéresse désormais à la résolution de l'équation

$$
\frac{\partial u}{\partial t}=\sum_{j=1}^{n} \frac{\partial}{\partial x_{j}}\left\{a_{j}(t, u, \nabla u)\right\}+f
$$

où $t, u, p \rightarrow a_{j}(t, u, p)$ est une fonction de $[0, T] \times \boldsymbol{R} \times \boldsymbol{R}^{n}$ dans $\boldsymbol{R}$ et on suppose que

(i) $a_{j}$ est continue et admet des dérivées secondes en u et $p$, continues en $t$ et localement lipschitziennes en $u$ et $p, j=1, \ldots, n$.

(ii) $\sum_{j, k=1}^{n} \frac{\partial a_{j}}{\partial p_{k}}(t, u, p) \xi_{j} \xi_{k}>0$ partout pour $\xi \neq 0$ dans $\boldsymbol{R}^{n}$.

Ceci étant, on a le

THÉORÈMe 7.12. - On suppose que les $a_{i}$ vérifient (7.6.) (i) et (ii). Soient $\left.p \in\right] n-1, \infty[$ et $s \in] \sup (0, n / p-1) ; 1 / p\left[\right.$. Alors pout tout $u_{0} \in h_{p}^{s+2}(\Omega)$ aveo $u_{0}=0$ sur $\Gamma$ et pour 
tout $f \in C\left([0, T] ; h_{p}^{s}(\Omega)\right)$, il existe $\tau>0$ et une unique $u \in O\left(\left[0, \tau\left[; h_{p}^{s+2}(\Omega)\right) \cap C^{1}([0, \tau[\right.\right.$; $\left.h_{p}^{s}(\Omega)\right)$ solution de l'équation (7.5.) vérifiant les conditions

$$
\left\{\begin{array}{l}
u(0, x)=u_{\theta}(x), \quad x \in \Omega \\
u(t, x)=0, \quad t \in[0, \tau[, \quad x \in \Gamma .
\end{array}\right.
$$

Df́monstration. - On conserve $A$ comme au début de 7.2. et on applique le Théorème 4.1. avec $\theta \in] 0,1 / 2 p\left[\right.$ avec toutefois $\theta>n / 2 p-\frac{1}{2}$. Dans ces conditions on a

$$
D_{\Lambda}(\theta)=h_{p}^{2 \theta}(\Omega)
$$

sans conditions aux limites et

$$
D_{\Lambda}(\theta+1)=\left\{u \in h_{p}^{2 \theta+2}(\Omega) ; u=0 \text { sur } \Gamma\right\} .
$$

On pose

$$
\varphi(t, u)=\sum_{j=1}^{n} \frac{\partial}{\partial x_{j}}\left\{a_{j}(t, u, \nabla u)\right\}+f .
$$

Comme on a supposé $s+1=2 \theta+1>n / p, h_{p}^{s+1}(\Omega)$ est une algèbre d'après le Lemme 7.9. $\left.{ }^{(15}\right)$ et d'après le Lemme 7.8. l'application.

$$
u \rightarrow a_{i}(t, u, \nabla u)
$$

est continue de $h_{p}^{s+2}(\Omega)$ dans $h_{p}^{s+1}(\Omega)$; on en déduit facilement (4.1.) (i).

Ensuite on démontre (4.1.) (ii) et l'identité

$$
\frac{\partial \varphi}{\partial u}(t, u) v=\sum_{j=1}^{n} \frac{\partial}{\partial x_{j}}\left\{\frac{\partial a_{j}}{\partial u}(t, u, \nabla u) v+\sum_{k=1}^{n} \frac{\partial a_{j}}{\partial p_{k}}(t, u, \nabla u) \frac{\partial v}{\partial x_{k}}\right\}
$$

toujours grâce aux mêmes lemmes.

De (7.6.) (ii), utilisant AGMON [1], on vérifie que pour $\lambda>0$ assez grand, l'opérateur différentiel $(\partial \varphi / \partial u)(t, u)$ est générateur infinitésimal d'un semi-groupe analytique fortement continu dans $E$ avec domaine $D_{A}$. Ensuite des résultats de régularité de Agmon-Douglis-Nirenberg [2], on déduit par interpolation que si $v \in D_{A}$ et

$$
\frac{\partial \varphi}{\partial u}(t, u) v \in D_{A}(\theta)=h_{p}^{2 \theta}(\Omega)
$$

alors $v \in h_{p}^{2 \theta+2}(\Omega)$ donc $v \in D_{A}(\theta+1)$. L'ensemble de l'hypothèse (4.1.) est ainsi vérifié; le résultat annoncé suit par application du Théorème 4.1.

(15) On s'est limité dans le Lemme 7.9. au cas $s<1$, cependant $h_{p}^{s}(\Omega)$ est une algèbre pour tout $s>n / p$. On peut le prouver en suivant la méthode indiqée dans le Lemme 7.9. C'est aussi (par densité des fonctions régulières) une conséquence de ZoLésio [23]. 
Remarque 7.13. - Les considérations ci-dessus sont également valables pour des équations de la forme

$$
\frac{\partial u}{\partial t}=\Delta a(u)+f
$$

Rimarqua 7.14. - Dans le Théorème 7.12. l'avantage est qu'on n'impose aucune condition aux limites sur les données $f$ et $\Delta u_{0}$. Ceci est intéressant même dans le cas où l'espace est de dimension $n=1$, situation envisagée en 7.1., car on ne peut pas faire disparaitre ces conditions aux limites superflues en restant dans le cadre de l'espace $E$ des fonctions continues (ceci ne concerne bien sûr, que les conditions aux limites de Dirichlet).

7.4. On va maintenant exploiter les résultats du $\S 5$ qui fournissent l'existence globale. On considère ici l'équation autonome à une variable d'espace

$$
\frac{\partial u}{\partial t}=a\left(\frac{\partial^{2} u}{\partial x^{2}}\right)
$$

où on supposera que $a$ est une fonction numérique réelle quatre fois continûment dérivable définie dans $\boldsymbol{R}$ et telle que

$$
\left\{\begin{array}{l}
a(0)=0, \quad a^{\prime}(x)>0 \quad \text { pour tout } x \in \boldsymbol{R} \\
a^{n}(0)=0
\end{array}\right.
$$

Notre but est ici d'appliquer le résultat synthétisé dans la Remarque 5.3. dont il faudra donc vérifier les (très nombreuses) hypothèses. On aurait pur traiter de la même manière une équation de la forme (7.1.) par contre il n'est pas clair qu'on pourrait résoudre de la même façon les équations à plusieurs variables d'espace du type de (7.2.).

Ici comme en 7.1. on a toujours

$$
\begin{gathered}
E=\{v \in O([0,1]) ; v(0)=v(1)=0\} \\
D_{A}=\left\{v \in C^{2}([0,1]) ; v(0)=v(1)=v^{\prime \prime}(0)=v^{\prime \prime}(1)=0\right\}
\end{gathered}
$$

et $A=d^{2} / d x^{2}$. Par conséquent

$$
\begin{aligned}
& D_{A}(\theta)=\left\{v \in h_{\infty}^{2 \theta}([0,1]) ; v(0)=v(1)=0\right\}, \quad 0<\theta<1, \\
& D_{A}(\theta)=\left\{v \in h_{\infty}^{2 \theta}([0,1]) ; v(0)=v^{\prime \prime}(0)=v(1)=v^{\prime \prime}(1)=0\right\}, \quad 1<\theta<2, \\
& D_{A}(\theta)=\left\{v \in h_{\infty}^{2 \theta}([0,1]) ; v(0)=v^{\prime \prime}(0)=v^{(i v)}(0)=v(1)=v^{\prime \prime}(1)=v^{(\mathrm{iv})}(1)=0\right\},
\end{aligned}
$$

si $2<0<3$. 
On vérifie maintenant l'hypothèse (i) de la Remarque 5.3. il est clair que si on pose $\varphi(u)=a\left(d^{2} u / d x^{2}\right)$, on a

$$
\varphi \in C^{1}\left(h_{\infty}^{s+2}([0,1]) ; h_{\infty}^{s}([0,1])\right)
$$

pour $s<3$ et alors

$$
\varphi^{\prime}(u) \cdot v=a^{\prime}\left(\frac{d^{2} u}{d x^{2}}\right) \frac{d^{2} v}{d x^{2}}
$$

Il faut cependant vérifier que $\varphi$ opère de $D_{A}(\theta+1)$ dans $D_{A}(\theta)$, c'est-z̀-dire que $a\left(u^{\prime \prime}\right)=0{ }^{(15)}$ en $x=0$ et 1 dès que $u=u^{\prime \prime}=0$ (ce qui résulte de l'hypothèse $\left.a(0)=0\right)$ et que $\left(a\left(u^{n}\right)\right)^{n}=0$ en $x=0$ et 1 dès que $u=u^{n}=u^{(\text {iv })}=0$ en ces points. Pour cela on calcule

$$
\left(a\left(u^{\prime \prime}\right)\right)^{\prime \prime}=\left(a^{\prime}\left(u^{\prime \prime}\right) u^{\prime \prime \prime}\right)^{\prime}=a^{\prime}\left(u^{\prime \prime}\right) u^{\prime \prime \prime}+a^{\prime \prime}\left(u^{\prime \prime}\right)\left(u^{\prime \prime \prime}\right)^{2} .
$$

On a done en 0 et 1

$$
\left(a\left(u^{\prime \prime}\right)\right)^{\prime \prime}=a^{\prime \prime}(0)\left(u^{\prime \prime \prime}\right)^{2}
$$

et c'est nul car on a supposé que $a^{\prime \prime}(0)=0$.

En conclusion on a prouvé que

$$
\varphi \in C^{1}\left(D_{A}(\theta+1) ; D_{A}(\theta)\right)
$$

pour que $0<2 \theta<3(2 \theta$ non entier).

Ensuite pour vérifier l'hypothèse (ii) on considère l'opérateur différentiel $\varphi^{\prime}(u)$ c'est-à-dire $a^{\prime}\left(u^{\prime \prime}\right)\left(d^{2} / d x^{2}\right)$ dans $E$. L'hypothèse $a^{\prime}(x)>0$ pour tout $x \in \boldsymbol{R}$, implique pour $u \in h_{\infty}^{s+2}([0,1])$ que $a^{\prime}\left(u^{\prime \prime}\right)$ admet une borne inférieure strictement positive, alors si on pose $\overline{p^{\prime}(u)}=a^{\prime}\left(u^{\prime \prime}\right)\left(d^{2} / d x^{2}\right)$ et $D_{q^{\tau}(u)}=D_{A}$, il résulte $d u$ Lemme 7.3. que $\overline{p^{\prime}(u)}$ est le générateur infinitésimal d'un semi-groupe analytique fortement continu dans $E$.

Par définition on a pour $0<0<1$,

$$
\begin{gathered}
D_{\varphi^{\prime}(u)}(\theta+1)=\left\{v \in D_{\Lambda} ; \widetilde{\varphi^{\prime}(u)} v \in D_{\Lambda}(\theta)\right\}=\left\{v \in 0^{2}([0,1]) ; a^{\prime}\left(u^{\prime \prime}\right) v^{\prime \prime} \in h_{\infty}^{2 \theta}([0,1]),\right. \\
\left.v(0)=v(1)=v^{\prime \prime}(0)=v^{\prime \prime}(\mathbf{1})=a^{\prime}\left(u^{\prime \prime}(0)\right) v^{\prime \prime}(0)=a^{\prime}\left(u^{\prime \prime}(1)\right) v^{\prime \prime}(\mathbf{1})=0\right\} .
\end{gathered}
$$

Alors si $u \in D_{A}(\theta+1), a^{\prime}\left(u^{\prime \prime}\right) \in h_{\infty}^{2 \theta}([0,1])$ et ne s'annule pas done $1 / a^{\prime}\left(u^{\prime \prime}\right) \in h_{\infty}^{2 \theta}([0,1])$ puisque cet espace est une algèbre; on en déduit que

$$
D_{\bar{\varphi}^{\prime}(v)}(\theta+1)=\left\{v \in h_{\infty}^{2 \theta+2}([0,1]) ; v(0)=v(1)=v^{\prime \prime}(0)=v^{\prime \prime}(1)=0\right\}=D_{\Lambda}(\theta+1) .
$$

${ }^{\left({ }^{16}\right)}$ Dans toute la suitè désigne la dérivation par rapport à $x$. 
Enfin pour $1<\theta<2$ on a par définition

$$
\begin{aligned}
& D_{r^{\prime}(u)}(\theta+1)=\left\{v \in D_{A} ; \widetilde{\varphi^{\prime}(u)} v\right.\left.\in D_{A}(\theta)\right\}=\left\{v \in C^{2}([0,1]) ; a^{\prime}\left(u^{\prime \prime}\right) v^{\prime \prime} \in h_{\infty}^{2 \theta}([0,1]),\right. \\
&\left.v(0)=v(1)=v^{\prime \prime}(1)=\left[a^{\prime}\left(u^{\prime \prime}\right) v^{\prime \prime}\right]^{\prime \prime}(0)=\left[a^{\prime}\left(u^{\prime \prime}\right) v^{\prime \prime}\right]^{\prime \prime}(1)=0\right\} .
\end{aligned}
$$

Si $u \in D_{\Lambda}(\theta+1)$ on a $a^{\prime}\left(u^{\prime \prime}\right) \in h_{\infty}^{2 \theta}([0,1])$ done la condition $a^{\prime}\left(u^{\prime \prime}\right) v^{\prime \prime} \in h_{\infty}^{2 \theta}([0,1])$ équivaut à $v^{\prime \prime} \in h_{\infty}^{2 \theta}([0,1])$ done à $v \in h^{20+2}([0,1])$. Ensuite on a

$$
\begin{aligned}
{\left[a^{\prime}\left(u^{\prime \prime}\right) v^{\prime \prime}\right]^{\prime \prime}(0)=} & {\left[a^{\prime}\left(u^{\prime \prime}\right) v^{\prime \prime \prime}+a^{\prime \prime}\left(u^{\prime \prime}\right) u^{\prime \prime \prime} v^{\prime \prime}\right]^{\prime}(0)=} \\
& =\left[a^{\prime}\left(u^{\prime \prime}\right) v^{\prime \prime \prime}+2 a^{\prime \prime}\left(u^{\prime \prime}\right) u^{\prime \prime \prime} v^{\prime \prime \prime}+a^{\prime \prime}\left(u^{\prime \prime}\right) u^{\prime \prime \prime} v^{\prime \prime}+a^{\prime \prime \prime}\left(u^{\prime \prime}\right)\left(u^{\prime \prime \prime}\right)^{2} v^{\prime \prime}\right](0)=a^{\prime}(0) v^{\prime \prime \prime}(0)
\end{aligned}
$$

puisque $u^{\prime \prime}(0)=0$ et $a^{\prime \prime}(0)=0$ par hypothèse. La condition $\left[a^{\prime}\left(u^{\prime \prime}\right) v^{\prime \prime}\right]^{\prime \prime}(0)=0$ équivaut donc à $v^{\prime \prime \prime \prime}(0)=0$. Le même raisonnement est valable au point $x=1$. A ce point, on a done établi l'identité

$$
\left.D_{\varphi^{\prime}(u)}(\theta+1)=\left\{v \in h_{\infty}^{2 \theta+2}([0,1]]\right) ; v(0)=v^{\prime \prime}(0)=v^{\prime \prime \prime}(0)=v(\mathbf{1})=v^{\prime \prime}(1)=v^{\prime \prime \prime}(\mathbf{1})=0\right\}
$$

done $D_{\varphi^{\prime}(u)}(\theta+1)=D_{A}(\theta+1)$.

On a ainsi complètement vérifié l'hypothèse (iii) de la Remarque 5.3.

L'hypothèse (iv) revient à voir que $u \mapsto a\left(u^{\prime \prime}\right)$ est une application bornée de $h_{\infty}^{s+2}([0,1])$ dans $h_{\infty}^{s}([0,1])$ pour $0<s<1$ ce qui est évident.

L'hypothèse (v) signifie que $u \mapsto a\left(u^{\prime \prime}\right)$ est inversible de

$$
\left\{u \in h_{\infty}^{s+2}([0,1]) ; u(0)=u^{\prime \prime}(0)=u^{(\mathrm{iv})}(0)=u(1)=u^{\prime \prime}(1)=u^{(\mathrm{iv})}(1)=0\right\}
$$

$\operatorname{sur}$

$$
\left\{f \in h_{\infty}^{s}([0,1]) ; f(0)=f^{\prime \prime}(0)=f(1)=f^{\prime \prime}(1)=0\right\}
$$

pour $2<s<3$. Il est évident que

$$
\varphi^{-1}(f)=\Lambda^{-1} a^{-1}(f)
$$

qui a un sens puisque par hypothèse $a$ est strictement croissante donc inversible, et son inverse $a^{-1}$ est de classe $C^{4}$; donc pour $f \in h_{\infty}^{s}([0,1])$ on a $a^{-1}(f) \in h_{\infty}^{s}([0,1])$ et $u=\Lambda^{-1} a^{-1}(f) \in h_{\infty}^{s+2}([0,1])$; de plus on a $u(0)=u(1)=0$ par définition de $\Lambda^{-1}$. Il reste à vérifier que $u=u^{\prime \prime}=0$ en $x=0$ et 1 .

On a $u^{\prime \prime}=u=a^{-1}(f)$ done $u^{\prime \prime}(0)=a^{-1}(f(0))=a^{-1}(0)=0$ et de même $u^{\prime \prime}(1)=0$, puisque

$$
u^{(\mathrm{iv})}=\left(a^{-1}(f)\right)^{\prime \prime}=\left(a^{-1}\right)^{\prime}(f) f^{\prime \prime}+\left(a^{-1}\right)^{\prime \prime}(f)\left(f^{\prime}\right)^{2}
$$

donc en zéro et un on a

$$
u^{(\mathrm{iv})}=\left(a^{-1}\right)^{\prime \prime}(0) f^{\prime}(0)^{2}=0
$$


puisque $a^{\prime \prime}(0)=0$. Ceci achève de prouver l'inversibilité de $\varphi$. Il est évident sur l'expression explicite de $\varphi^{-1}$ que e'est une application continue.

A ce point, toutes les hypothèses de la Remarque 5.3. sont vérifiées, sauf l'estimation a priori. On doit done considérer à présent $u_{0} \in h_{\infty}^{s+4}([0,1])$ avec $0<s<1$ vérifiant $u_{0}=u_{0}^{\prime \prime}=u_{0}^{(\mathrm{iv})}=0$ en 0 et 1 et

$$
u \in C\left(\left[0, \tau\left[; h_{\infty}^{s+4}([0,1])\right) \cap C^{1}\left(\left[0, \tau\left[; h_{\infty}^{s+2}([0,1])\right.\right.\right.\right.\right.
$$

solution de (7.7.) avec $u(0, x)=u_{0}(x)$ et $u(t, 0)=u(t, 1)=0$ et on doit prouver que $u$ est bornée à valeurs dans $h_{\infty}^{\sigma+2}([0,1])$ avec un $\sigma>s$.

On sait done que

$$
\frac{\partial^{\alpha} u}{\partial x^{\alpha}}, \quad 0 \leqslant \alpha \leqslant 4 \quad \text { et } \quad \frac{\partial^{\beta}}{\partial x^{\beta}} \frac{\partial u}{\partial t}, \quad 0 \leqslant \beta \leqslant 2
$$

sont continues dans $[0, \tau[\times[0,1]$ et si on choisit $\sigma<1$, ce qui est loisible, il suffit de vérifier que

$$
\underset{\substack{x \in[0,1] \\ l \in[0, \tau \llbracket}}{\operatorname{Max}}\left|\frac{\partial \gamma u}{\partial x^{\gamma}}(t, x)\right| \leqslant M, \quad 0 \leqslant \gamma \leqslant 3 .
$$

En premier le principe du maximum s'applique à l'équation (7.7.) et par conséquent

$$
\operatorname{Max}_{x \in[0,1]}|u(t, x)| \leqslant \operatorname{Max}_{x \in[0,1]}\left|u_{0}(x)\right|
$$

pour $t \in[0, \tau[$.

Ensuite tenant compte de la régularité de $u$ indiquée en (7.9.) on voit qu'il est possible de dériver l'équation (7.7.) par rapport au temps et on obtient ainsi l'équation de $v=\partial u j \partial t$ qui suit

$$
\frac{\partial v}{\partial t}=a^{\prime}\left(u^{\prime \prime}\right) \frac{\partial^{2} v}{\partial x^{2}}
$$

avec $v(0, x)=a\left(u_{0}^{\prime \prime}(x)\right)$ et $v(t, x)=0$ pour $x=0$ ou 1 . Une nouvelle application $d u$ principe du maximum montre que

$$
\underset{x \in[0,1]}{\operatorname{Max}}|v(t, x)| \leqslant \underset{x \in[0,1]}{\operatorname{Max}}\left|a\left(u_{0}^{\prime \prime}(x)\right)\right|
$$

Comme $u=\Lambda^{-1} a^{-1}(v)$ on en déduit que $u^{\prime \prime}$ reste borné et partant il existe $C$ tel que

$$
\left|u^{\prime \prime}(t, x)\right| \leqslant C
$$

pour $t \in[0, \tau[$ et $x \in[0,1]$. 
On conclut à l'aide du

LEMME 7.15. - Soit a une fonction continue strictement positive dans $[0, \tau[\times[0,1]$ et $v$ solution deux fois continûment dérivable en $x$ de l'équation

$$
\frac{\partial v}{\partial t}=\alpha \frac{\partial^{2} v}{\partial x^{2}}
$$

avec $v(0, x)=v_{0}(x)$ et $v(t, 0)=v(t, \mathbf{1})=0$ pour tout $t \in[0, \tau[$, alors

$$
\underset{x \in[0,1]}{\operatorname{Max}}\left|\frac{d v}{d x}(t, x)\right| \leqslant \operatorname{Max}_{x \in[0,1]}\left|v_{0}^{\prime}(x)\right|
$$

pour tout $t \in[0, \tau[$.

Démonstration. - On approche $\alpha$ par des fonctions $\alpha_{n}$ continûment dérivables strictement positives et $v_{0}$ par $v_{0, n}$ trois fois continûment dérivable. La solution $v_{n}$ correspondante des équations

$$
\frac{\partial v_{n}}{\partial t}=\alpha_{n} \frac{\partial^{2} v_{n}}{\partial x^{2}}, \quad v_{n}(0, x)=v_{0, n}(x), \quad v_{n}(t, 0)=v_{n}(t, 1)=0
$$

converge lorsque $n \rightarrow+\infty$, vers $v$. Les $v_{n}$ étant trois fois continûment dérivables en $x$ on peut écrire l'équation de $w_{n}=\partial v_{n} / \partial x$ soit

$$
\frac{\partial w_{n}}{\partial t}=\alpha_{n} \frac{\partial^{2} w_{n}}{\partial x^{2}}+\frac{\partial \alpha_{n}}{\partial x} \frac{\partial w_{n}}{\partial x}
$$

avee les conditions $\left(d w_{n} / d x\right)(t, 0)=\left(d w_{n} / d x\right)(t, 1)=0$ et la condition initiale $w_{n}(0, x)=$ $=v_{0, n}^{\prime}(x)$. Le principe du maximum appliqué à $w_{n}$ donne la majoration

$$
\underset{x \in[0,1]}{\operatorname{Max}}\left|w_{n}(t, x)\right| \leqslant \underset{x \in[0,1]}{\operatorname{Max}}\left|v_{0, n}^{\prime}(x)\right| .
$$

On en déduit (7.11.) par passage à la limite. C.Q.F.D.

En conclusion, on a établi le

THÉOR亡̀m 7.16. - Soit a une fonction numérique quatre fois dérivable définie dans $\boldsymbol{R}$ avee

$$
a(0)=a^{\prime \prime}(0)=0
$$

et $a^{\prime}(x)>0$ pour tout $x \in \boldsymbol{R}$ et soit $0<s<1$ et $u_{0} \in h_{\infty}^{4+s}([0,1])$ telle que $u_{0}=u_{0}^{u}=u_{0}^{(\mathrm{iv})}=0$ en $x=0$ et $x=1$. Alors il existe une unique $u \in O\left(\left[0, \infty\left[; h_{\infty}^{4+s}([0,1])\right) \cap C^{1}([0, \infty[\right.\right.$; $\left.h_{\infty}^{2+8}([0,1])\right)$ solution de

$$
\frac{\partial u}{\partial t}=a\left(\frac{\partial^{2} u}{\partial x^{2}}\right)
$$

aveo les conditions $u(0, x)=u_{0}(x)$ et $u(t, 0)=u(t, 1)=0$. 


\section{8. - Problèmes mixtes (Ordre Supérieur).}

Les équations considérées dans le $\$ 7$ sont susceptibles d'être résolues dans des espaces voisins de ceux considérés ici par des méthodes utilisant à la base le principe du maximum comme dans BrUCE-STEwart [6], BenILAN-HA [3]. On va étudier ici un exemple de problème mixte non linéaire pour une équation parabolique d'ordre quatre qui ne peut pas être résolue à l'aide du principe du maximum. Cet exemple n'a pas la prétention d'être exhaustif.

Il s'agit de l'équation

$$
\frac{\partial u}{\partial t}=a\left(\Delta^{2} u\right)+f
$$

où a est une fonction continûment dérivable et de dérivée première $a^{\prime}$ localement lipschitzienne.

Théonème 8.1. - On suppose que $a^{\prime}(x)<0$ pour tout $x \in \boldsymbol{R}$ et que $a(0)=0$. Soit $p \in] n, \infty[$ et $s \in] n / p, 1\left[\right.$. Alors pour tout $u_{0} \in h_{p}^{s+4}(\Omega)$ avec $u_{0}=\partial u_{0} / \partial v=\Delta^{2} u_{0}=0 \mathrm{sur} T$ et pour tout $f \in C\left([0, T] ; h_{p}^{8}(\Omega)\right)$ tel que $f(t, x)=0$ pour $t \in[0, T]$ et $x \in T$, il existe $\tau>0$ et une unique

$$
u \in C\left([0, T] ; h_{p}^{s+4}(\Omega)\right) \cap C^{1}\left([0, T] ; h_{p}^{s}(\Omega)\right)
$$

solution de $(8, \mathbf{1})$ et vérifiant les conditions

$$
\left\{\begin{array}{l}
u(0, x)=u_{0}(x), \quad x \in \Omega \\
u(t, x)=0, \quad t \in[0, \tau], \quad x \in \Gamma \\
\frac{\partial u}{\partial v_{x}}(t, x)=0, \quad t \in[0, \tau], \quad x \in \Gamma .
\end{array}\right.
$$

Démonstration. - On va appliquer le Théorème 4.1. avec les choix suivants $E=L_{m}(\Omega), A=-\Delta^{2}$,

$$
D_{A}=\left\{u \in W_{p}^{4}(\Omega) ; u=\frac{\partial u}{\partial v}=0 \text { sur } \Gamma\right\} \quad \text { et } \quad \varphi(u)=a\left(\Delta^{2} u\right) .
$$

Toujours d'après Agmon [1] l'opérateur $A$ est le générateur infinitésimal d'un semi-groupe analytique vérifiant $(3,2)$, Le Théorème 6.1 . montre que

$$
D_{\Lambda}(\theta)=\left\{u \in h_{p}^{4 \theta}(\Omega) ; u=\frac{\partial u}{\partial v}=0 \text { sur } \Gamma\right\}
$$

si $4 \theta>1+1 / p$ et

$$
D_{\Lambda}(\theta)=\left\{u \in h_{p}^{4 \theta}(\Omega) ; u=0 \operatorname{sur} \Gamma\right\}
$$


si $1+1 / p>4 \theta>1 / p$ et enfin

$$
D_{\Lambda}(\theta)=h_{p}^{4 \theta}(\Omega)
$$

si $4 \theta<1 / p$.

On travaillera dans la suite avec $4 \theta=s \in] n / p, 1\left[\right.$ de sorte que $D_{A}(\theta)$ soit une algèbre grâce au Lemme 7.9. Ceci étant il est alors clair d'après AGMor-DougulsNiREnberg [2] que

$$
D_{\Lambda}(\theta+1)=\left\{u \in h_{s}^{s+4}(\Omega) ; u=\frac{\partial u}{\partial v}=\Delta^{2} u=0 \operatorname{sur} \Gamma\right\} .
$$

Ensuite on voit grâce au Lemme 7.8 . que

$$
\varphi \in O^{1}\left(D_{\Lambda}(\theta+1) ; h_{p}^{s}(\Omega)\right)
$$

et que

$$
\frac{\partial \varphi}{\partial u}(u) v=a^{i}\left(\Delta^{2} u\right) \Delta^{2} v
$$

On s'assure des mêmes propriétés à valeurs dans $D_{A}(\theta)$ grâce à l'hypothèse $a(0)=0$ qui fait que pour $u \in D_{A}\left(\theta+1\right.$ ) (donc $\Delta^{2} u=0$ sur $\Gamma$ ) on a $\varphi(u)=a\left(\Delta^{2} u\right)=0$ sur $\Gamma$.

Ensuite pour $u \in h_{p}^{s+4}(\Omega)$, on a $\Delta^{2} u \in h_{p}^{s}(\Omega) \subset W_{p}^{s-\varepsilon}(\Omega)$ pour tout $\varepsilon>0$; on peut choisir $\varepsilon$ tel que $s-\varepsilon>n / p$ et par conséquent on a $\Delta^{2} u \in C(\bar{\Omega})$ et done $a^{\prime}\left(\Delta^{2} u\right)$ est une fonction continue dont le maximum est strictement négatif puisque $a^{\prime}(x)<0$ pour tout $x$. Ceci implique toujours grâce à la théorie d'AGMoN [1] que l'opérateur différentiel $(\partial \varphi / \partial u)(u)-\lambda I$ est pour $\lambda$ assez grand (dépendant de $u)$ le générateur infinitésimal d'un semi-groupe analytique dans $E$ de domaine $D_{A}$.

Ensuite grâce à $\triangle$ GMON-Dodguls-NIreinberg [2] et à l'interpolation les conditions $v \in D_{A}$ arec $(\partial \varphi / \partial u)(u) v \in D_{A}(\theta) \subset h_{p}^{4 \theta}(\Omega)$ implique que $v \in h_{p}^{4 \theta+4}(\Omega)$ et aussi

$$
\Delta^{2} v=\frac{1}{a^{\prime}\left(\Delta^{2} u\right)} \frac{\partial \varphi}{\partial u}(u) v
$$

done $\Delta^{2} v=0$ sur $\Gamma$ ceci nous assure que

$$
D_{(\tilde{\partial} \varphi / \partial u)(u)-\lambda I}(\theta+1)=D_{\Lambda}(\theta+1) .
$$

On a ainsi vérifié toutes les hypothèses (4.1.) et par conséquent le Théorème 8.1. suit par application du Théorème 4.1.

Remarque 8.2. - Dans le cas de la dimension d'espace $n=1$, on peut comme en 7.1., travailler dans le cadre de l'espace $E=C([0,1])$. On obtient alors sans faire appel aux résultats du $\& 6$ que pour $\varrho \in] 0,1\left[, u_{0} \in h_{\infty}^{\varrho+4}([0,1])\right.$ avec $u_{0}(0)=$ 
$=u_{0}(1)=u_{0}^{\prime}(0)=u_{0}^{\prime}(1)=u^{(4)}(0)=u^{(4)}(1)=0$ et pour $f \in O\left([0, T] ; h_{\infty}^{\varrho}([0,1])\right)$ vérifiant $f(t, 0)=f(t, 1)$ pour tout $t \in[0, T]$, il existe $\tau>0$ et

$$
u \in C\left([0, \tau] ; h_{\infty}^{e+4}([0,1])\right) \cap C^{1}\left([0, \tau] ; h_{\infty}^{e}([0,1])\right)
$$

solution de

$$
\left\{\begin{array}{l}
\frac{\partial u}{\partial t}=a\left(\frac{\partial^{4} u}{\partial x^{4}}\right)+f, \quad t \in[0, \tau], \quad x \in[0,1] \\
u(0, x)=u_{0}(x), \quad x \in[0,1] \\
u(t, 0)=u(t, 1)=0, \quad t \in[0, \tau] \\
\frac{\partial u}{\partial x}(t, 0)=\frac{\partial u}{\partial x}(t, 1)=0, \quad t \in[0, \tau] .
\end{array}\right.
$$

REMARque 8.3. - On peut résoudre de la même manière les équations de la forme (8.1.) avee n'importe quel système de conditions aux limites qui fait de $A^{2}$ un générateur infinitésimal de semi-groupe analytique dans $L_{p}(\Omega)$.

Remarque 8.4. - Comme en 7.2., on peut supprimer les conditions aux limites insolites sur $\Delta^{2} u_{0}$ et sur $f$, nécessaires dans le Théorème 8.1. si on étudie (par exemple) des équations de la forme

$$
\frac{\partial u}{\partial t}=\Delta a(\Delta u)+f
$$

où $a$ est une fonction trois fois continûment dérivable de dérivée troisième localement lipschitzienne, vérifiant $a^{\prime}(x)<0$ pour tout $x$. Il suffit alors d'appliquer le Théorème 4.1 . avec $\theta=s / 4,1 / p>s>\operatorname{Max}(0, n / p-2)$ et alors $D_{A}(\theta)=h_{p}^{s}(\Omega)$ tandis que $h_{p}^{s+2}(\Omega)$ est une algèbre ce qui permet de vérifier les hypothèses (4.1.).

\section{BIBLIOGRAPHIE}

[1] AGMON, On the eigentunetions and on the eigenvalues of general elliptic boundary value problems, Comm. Pure Applied. Math., 15 (1962), p. 119.

[2] AgMon - Dovalis - NrRENBERG, Estimates near the boundary for solutions of elliptic partial differential equations, Comm. Pure Applied Math., 12 (1959), p. 623.

[3] Benilan-Ha, Equations d'évolution $d u$ type $(d u s / d t)+\beta \partial \varphi(u) \ni 0$ dans $L^{\infty}(\Omega)$, Note C.R.A.S., 281 (1975), pp. 947-950.

[4] BREZIs, Opérateurs maximaux monotones et semi-groupes de contractions dans les espaces de Hilbert, Math. Studies 5, North Holland, 1973.

[5] BREzIs - FraENKEL, A function with prescribed initial derivatives in different Banach spaces (à para"tre). 
396 Givsteppe Da Prato - PierRe Grisvard: Equations d'évolution abstraites, ete.

[6] Brdex - STewart, Generation of analytic semi-groups by strongly elliptic operators, Transactions Amer. Math. Soc., 199 (1977), pp. 141-162.

[7] CRANDALL - LigGETt, Generation of nonlinear transformations in generat Banach spaces, Amer. J. Math., 93 (1971), pp. 265-298.

[8] Crandall- PazY, Nonlinear evolution equations in B-spaces, Israël J. of Math., 11 (1972), pp. 57-94.

[9] Da Prato - GRISvard, Sommes d'opérateurs linéaires et équations différentielles opérahionnelles, J. de Maths. pures et appliquées, 54 (1975), pp. 305-387.

[10] Da Prato - GRISVARD, Equations d'évolution abstraites non linéaires de type parabolique, Note C.R.A.S., 283 (1976), pp. 709-711.

[11] Dunford - Schwartz, Linear operators, tomes 1 et 2, Intersciences Publishers, New York.

[12] Friedman, Remarks on non linear parabolic equations, Proc. Symp. Appl. Math., 17 (1965), pp. 297-350.

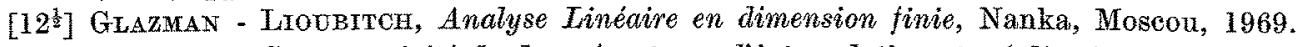

[13] Grisvard, Commutativité de deux foncteurs dinterpolation et aṕplications, J. de Maths. pures et appliquées, 45 (1966), pp. 143*290.

[13部] Grisvard, Equations différentielles abstraites, Ann. Sc. E.N.S., Paris, 2 (1969), pp. 311 -395.

[14] KaTo, Accretive operators and nonlinear evolution equations in B-spaces, Proc. Symp. A.M.S., 1968.

[15] KomurA, Nonlinear semi-groups in Hilbert spaces, J. Math. Soe. Japan, 19 (1967), pp. $493-507$.

[16] Lrons - Peerre, Sur une classe d'espaces d'interpolation, Publications I.H.E.S. Paris, 19 (1963), pp. 5-68.

[17] Mumu, Sur les multiplicateurs des intégrales de Fourier, Doklady Akad. Nauk. S.S.S.R., 109, no. 4 (1956), pp. 701-703.

[18] NIKoLski, Sur les théorèmes d'immersion, de prolongement et d'approximation pou les fonctions différentiable sde plusieurs variables, Uspehi Mat. Nauk., 16 (1961), pp. 63-114.

[19] PAZY, Semi-groups of linear operators and applications to partial differential equations, Lecture Notes no. 10, University of Maryland, 1974.

[20] Sinestrari - Vernole, Semi linear evolution equations in interpolation space. Non Linear Analysis, 1, no. 3 (1977), pp. 249-261.

[21] Soвotevski, Equations of parabolic type in a Banach space, Trudy Mockovkogo Mat. Obsch., 10 (1961), pp. 297-350.

[22] STEIN, Singular integrals and aifferentiability properties of functions, Princeton University Press, 1970.

[23] ZoLEsio, Multiplications dans les espaces de Besov, Proceedings of the Royal Academy of Scotland. 